TO APPEAR IN THE ASTROPHYSICAL JOURNAL

Preprint typeset using LTEX style emulateapj v. 08/22/09

\title{
A PREDICTION FORMULA OF SUPERSOFT X-RAY PHASE OF CLASSICAL NOVAE
}

\author{
IZUMI HACHISU \\ Department of Earth Science and Astronomy, College of Arts and Sciences, University of Tokyo, Komaba, Meguro-ku, Tokyo 153-8902, Japan \\ AND \\ MARIKO KATO \\ Department of Astronomy, Keio University, Hiyoshi, Kouhoku-ku, Yokohama 223-8521, Japan \\ to appear in the Astrophysical Journal
}

\begin{abstract}
On the basis of the recently developed universal decline law of classical novae, we propose prediction formulae for supersoft $\mathrm{X}$-ray on and off times, i.e., $t_{\mathrm{X}-\mathrm{on}}=(10 \pm 1.8) t_{3}$ days and $t_{\mathrm{X}-\mathrm{off}}=(5.3 \pm 1.4)\left(t_{3}\right)^{1.5}$ days for $8 \lesssim t_{3} \lesssim 80$ days. Here $t_{3}$ is the newly proposed "intrinsic" decay time during which the brightness drops by 3 magnitudes from optical maximum along our universal decline law fitted with observation. We have determined the absolute magnitude of our free-free emission model light curves and derived maximum magnitude vs. rate of decline (MMRD) relations. Our theoretical MMRD relations are governed by two parameters, one is the white dwarf (WD) mass and the other is the initial envelope mass at a nova outburst; this second parameter explains the scatter of MMRD points of individual novae. Our theoretical MMRD relations are also in good agreement with the well-known empirical formulae. We also show another empirical relation of $M_{V}(15) \sim-5.7 \pm 0.3$ based on the absolute magnitude of our model light curves, i.e., the absolute magnitude at 15 days after optical maximum is almost common among various novae. We analyzed ten nova light curves, in which a supersoft X-ray phase was detected, and estimated their WD masses. The models best reproducing simultaneously the optical and supersoft X-ray observations are ONeMg WDs with $1.28 \pm 0.04 M_{\odot}$ (V598 Pup), $1.23 \pm 0.05 M_{\odot}(\mathrm{V} 382 \mathrm{Vel}), 1.15 \pm 0.06 M_{\odot}(\mathrm{V} 4743 \mathrm{Sgr}), 1.13 \pm 0.06 M_{\odot}(\mathrm{V} 1281 \mathrm{Sco}), 1.2 \pm 0.05 M_{\odot}$ (V597 Pup), $1.06 \pm 0.07 M_{\odot}(\mathrm{V} 1494 \mathrm{Aql}), 1.04 \pm 0.07 M_{\odot}(\mathrm{V} 2467 \mathrm{Cyg}), 1.07 \pm 0.07 M_{\odot}(\mathrm{V} 5116 \mathrm{Sgr})$, $1.05 \pm 0.05 M_{\odot}$ (V574 Pup), and a CO WD with $0.93 \pm 0.08 M_{\odot}$ (V458 Vul). The newly proposed relationships are consistent with the emergence or decay epoch of the supersoft X-ray phase of these ten novae. Finally, we discuss the mechanism of shock-origin hard X-ray component in relation to the emergence of companion star from the WD envelope.
\end{abstract}

Subject headings: novae, cataclysmic variables — stars: mass loss — white dwarfs — X-rays: stars

\section{INTRODUCTION}

Classical novae are a thermonuclear runaway event on a white dwarf (WD) in a binary system, in which the WD accretes hydrogen-rich matter from the companion star. When the accreted matter reaches a critical value, hydrogen at the bottom of the WD envelope ignites to trigger a shell flash (denoted by time $t_{\mathrm{OB}}$ ). Just after a nova outburst, the envelope on the WD rapidly expands to a giant size and optically thick winds blow. The WD envelope settles in a steady-state. The optical magnitude attains the peak at the maximum expansion of the photosphere (time $t_{0}$ ). Then the photosphere gradually shrinks whereas the total luminosity is almost constant during the outburst. Thus, the photospheric temperature $T_{\mathrm{ph}}$ increases with time. The main emitting wavelength region moves from optical to ultraviolet and finally to supersoft X-ray, corresponding to from $T_{\mathrm{ph}} \sim 10^{4} \mathrm{~K}$ through $\sim 10^{6} \mathrm{~K}$. The optically thick winds blow continuously from the very beginning of the outburst until the photospheric temperature increases to $\log T_{\mathrm{ph}}(\mathrm{K}) \sim 5.4$. Just when the optically thick wind stops (time $t_{\text {wind }}$ ), the temperature quickly rises and the supersoft X-ray phase starts (e.g., Kato \& Hachisu 1994).

Thus, classical novae become a transient supersoft X-ray source in a later phase of the outburst, but their X-ray detection is rather rare mainly because of sparse observing time of X-ray satellites (e.g., Krautter et al. 1996; Orio et al. 2001a;

Electronic address: hachisu@ea.c.u-tokyo.ac.jp

Electronic address: marıko@educ.cc.ke1o.ac.jp
Ness et al. 2007a). If there is a formula to predict a supersoft $\mathrm{X}$-ray phase of novae, we can plan more efficient observation. The aim of this paper is to propose such a formula.

Novae show a rich variety of light curves; the timescales of decline, shapes, and total durations of outbursts are very different from nova to nova. Therefore, it is difficult to predict a later supersoft X-ray phase only from the early optical light curve. Recently we have proposed the universal decline law of classical novae (Hachisu \& Kato 2006, 2007; Hachisu et al. 2008), i.e., there is a basic light curve which can basically follow (or overlap with) individual nova light curves by squeezing or stretching it in the direction of time. We call this "a universal decline law." The universal shape of light curves is almost independent of the WD mass and of the chemical composition of its hydrogen-rich envelope. This implies that each nova light curve can be uniquely specified by one parameter (timescaling factor - squeezing/stretching factor against a universal shape of nova light curves).

We have reproduced a number of light curves of novae from early phase to supersoft X-ray phase (turn-on/turnoff times) (Hachisu \& Kato 2006, 2007; Hachisu et al. 2006, 2007, 2008). Based on these experiences we are now able to construct empirical formulae that tell us the emergence/decay times of a supersoft X-ray phase of individual novae from observational information of the early phase. We hope that these formulae are useful for future X-ray observations of classical novae.

The next section (Section 2) describes our numerical 
method for obtaining X-ray, ultraviolet (UV), optical, and infrared (IR) light curves. In Section 3, we show homologous shapes of our model light curves. Using characteristic properties of free-free emission model light curves, we derive the absolute magnitude of each optical light curve, which provides a unique standard candle of novae. We further propose another method for obtaining the nova absolute brightness by fitting a nova light curve with the other the brightness of which is known. After various nova timescales and relations between these timescales are presented, we propose our prediction formulae of a supersoft X-ray phase in Section 4. Then, we present our theoretical understanding on the so-called maximum magnitude vs. rate of decline (MMRD) relation of classical novae in Section 5. The light-curve analyses of ten classical novae are presented in Section 6 . The X-ray turnon/turnoff times of these ten novae, i.e., V598 Pup, V382 Vel, V4743 Sgr, V1281 Sco, V597 Pup, V1494 Aql, V2467 Cyg, V5116 Sgr, V574 Pup, and V458 Vul, are consistent with the above relations (formulae). Conclusions follow in Sections 7

\section{MODELING OF NOVA LIGHT CURVES}

The decay phase of novae can be followed by a sequence of steady state solutions (e.g., Kato \& Hachisu 1994). Using the same method and numerical techniques as in Kato \& Hachisu (1994), we have followed evolutions of novae by connecting steady state solutions along the decreasing envelope mass sequence. The mass of the hydrogen-rich envelope is decreasing due to wind mass-loss and nuclear burning. We solve a set of equations, consisting of the continuity, equation of motion, radiative diffusion, and conservation of energy, from the bottom of the hydrogen-rich envelope through the photosphere assuming spherical symmetry. Winds are accelerated deep inside the photosphere so that they are called "optically thick winds."

We have presented a unified model for IR, optical, UV, and supersoft X-ray light curves of the decay phase of classical novae (Hachisu \& Kato 2006, 2007; Hachisu et al. 2007, 2008; Kato \& Hachisu 2007). The optical and IR luminosity are reproduced by free-free emission from optically thin plasma (e.g., Gallagher \& Ney 1976; Krautter et al. 1984). This free-free emission flux at the frequency $\nu$ is estimated from Equation (9) of Hachisu \& Kato (2006), i.e.,

$$
F_{\nu} \propto \frac{\dot{M}_{\mathrm{wind}}^{2}}{v_{\mathrm{ph}}^{2} R_{\mathrm{ph}}},
$$

where $\dot{M}_{\text {wind }}$ is the wind mass-loss rate, $v_{\text {ph }}$ the wind velocity at the photosphere, and $R_{\mathrm{ph}}$ the photospheric radius, all of which are taken from our wind solutions. It should be noted that the shapes of free-free emission light curves are independent of the frequency (wavelength). After the optically thick wind stops, the total mass of the ejecta remains constant in time. The flux from such homologously expanding ejecta is estimated from Equation (19) of Hachisu \& Kato (2006), i.e.,

$$
F_{\nu} \propto t^{-3}
$$

where $t$ is the time after the outburst.

Assuming a blackbody spectrum with the photospheric temperature of $T_{\text {ph }}$, we have calculated the UV $1455 \AA$ light curve for a narrow energy band of 1445-1465 $\AA$ (see, e.g., Cassatella et al. 2002; Hachisu \& Kato 2006, for more details) and have also estimated the supersoft X-ray flux (Kato \& Hachisu 1994; Hachisu \& Kato 2006) throughout
TABLE 1

Chemical Composition OF White DWARF ENVELOPE

\begin{tabular}{lcclll}
\hline \hline nova case & $X$ & $Y$ & $X_{\mathrm{CNO}}$ & $X_{\mathrm{Ne}}$ & $Z^{\mathrm{a}}$ \\
\hline CO nova 1 & 0.35 & 0.13 & 0.50 & 0.0 & 0.02 \\
CO nova 2 & 0.35 & 0.33 & 0.30 & 0.0 & 0.02 \\
CO nova 3 & 0.45 & 0.18 & 0.35 & 0.0 & 0.02 \\
CO nova 4 & 0.55 & 0.23 & 0.20 & 0.0 & 0.02 \\
CO nova 5 & 0.65 & 0.27 & 0.06 & 0.0 & 0.02 \\
Ne nova 1 & 0.35 & 0.33 & 0.20 & 0.10 & 0.02 \\
Ne nova 2 & 0.55 & 0.30 & 0.10 & 0.03 & 0.02 \\
Ne nova 3 & 0.65 & 0.27 & 0.03 & 0.03 & 0.02 \\
Solar & 0.70 & 0.28 & 0.0 & 0.0 & 0.02 \\
\hline a carbon, nitrogen, oxygen, and neon are also included in
\end{tabular}

$Z=0.02$ with the same ratio as the solar abundance

the present paper for the energy range of $0.2-0.6 \mathrm{keV}$ (e.g., Hachisu \& Kato 2009). Even if we adopt a different energy range of $0.2-2.0 \mathrm{keV}$, shapes of supersoft X-ray light curves hardly change because the blackbody photosphere emits photons with energy of $<0.6 \mathrm{keV}$ for $M_{\mathrm{WD}} \lesssim 1.2 M_{\odot}$.

The light curves of our optically thick wind model are parameterized by the WD mass $\left(M_{\mathrm{WD}}\right)$, chemical composition of the WD envelope $\left(X, Y, X_{\mathrm{CNO}}, X_{\mathrm{Ne}}\right.$, and $\left.Z\right)$, and envelope mass $\left(M_{\mathrm{env}, 0}\right)$ at the time of the outburst $\left(t_{\mathrm{OB}}\right)$. Here $X$ is the hydrogen, $Y$ the helium, $X_{\mathrm{CNO}}$ the carbon-nitrogen-oxygen, $X_{\mathrm{Ne}}$ the neon, and $Z$ the metallicity content of the envelope. We adopt $Z=0.02$, which also includes carbon, nitrogen, oxygen, and neon with solar composition ratios. Table 1 shows nine sets of chemical compositions assumed in our models. The details of our model light curves and their parameter dependence are described in Hachisu \& Kato (2006).

\section{UNIVERSAL DECLINE LAW}

The decay timescale of a light curve depends mainly on the WD mass and weakly on the chemical composition, whereas the maximum brightness of a light curve depends strongly on the initial envelope mass, $M_{\mathrm{env}, 0}$. In this section, we present various features, especially the absolute magnitudes, of nova light curves based on our universal decline law.

\subsection{Template of nova light curves}

Figure 1 shows our free-free emission model light curves calculated from Equation (1), i.e.,

$$
m_{\mathrm{ff}}=-2.5 \log \left[\frac{\dot{M}_{\mathrm{wind}}^{2}}{v_{\mathrm{ph}}^{2} R_{\mathrm{ph}}}\right]_{(t)}^{\left\{M_{\mathrm{WD}}\right\}}+G^{\left\{M_{\mathrm{wD}}\right\}},
$$

for various WD masses, from $M_{\mathrm{WD}}=0.55$ to $1.2 M_{\odot}$ by step of $0.05 M_{\odot}$ for a chemical composition of "CO nova 2 " (these numerical data are tabulated in Table 2). The subscript $(t)$ explicitly expresses that this is a function of time while the superscript $\left\{M_{\mathrm{WD}}\right\}$ indicates a model parameter of WD mass. An optically thick wind phase continues for a long time, from a very early phase to a late phase of a nova outburst. In this figure we plot free-free emission model light curves calculated by Equation (3) from a very early phase until the end of an optically thick wind phase. The 15 th mag point of each light curve corresponds to the end of the wind phase. In other words, we define a constant of $G^{\left\{M_{\mathrm{wD}}\right\}}$ in Equation (3) as that the last (lowest) point of each light curve (the end of an optically thick wind phase) is 15 th mag. As a result, the value of $G^{\left\{M_{\mathrm{WD}}\right\}}$ is different for different $M_{\mathrm{WD}}$. In order to make Table 2 more compact, we have defined $G^{\left\{M_{\mathrm{WD}}\right\}}$ in this way. 
TABLE 2

Light CuRVES OF CO NOVAE ${ }^{\mathrm{a}}$

\begin{tabular}{|c|c|c|c|c|c|c|c|c|c|c|c|c|c|c|}
\hline $\begin{array}{c}m_{\mathrm{ff}} \\
(\mathrm{mag})\end{array}$ & $\begin{array}{c}0.55 M_{\odot} \\
\text { (day) }\end{array}$ & $\begin{array}{c}0.6 M_{\odot} \odot \\
\text { (day) }\end{array}$ & $\begin{array}{c}0.65 M_{\odot} \\
\text { (day) }\end{array}$ & $\begin{array}{c}0.7 M_{\odot} \\
\text { (day) }\end{array}$ & $\begin{array}{c}0.75 M_{\odot} \\
\quad \text { (day) }\end{array}$ & $\begin{array}{c}0.8 M_{\odot} \\
\text { (day) }\end{array}$ & $\begin{array}{c}0.85 M_{\odot} \odot \\
\text { (day) }\end{array}$ & $\begin{array}{c}0.9 M_{\odot} \\
\text { (day) }\end{array}$ & $\begin{array}{c}0.95 M_{\odot} \\
\text { (day) }\end{array}$ & $\begin{array}{c}1.0 M_{\odot} \\
\text { (day) }\end{array}$ & $\begin{array}{c}1.05 M_{\odot} \odot \\
\text { (day) }\end{array}$ & $\begin{array}{c}1.1 M_{\odot} \\
\text { (day) }\end{array}$ & $\begin{array}{c}1.15 M_{\odot} \\
\quad(\text { day })\end{array}$ & $\begin{array}{c}1.2 M_{\odot} \\
\text { (day) }\end{array}$ \\
\hline 1.50 & 0.000 & 0.000 & 0.000 & 0.000 & 0.000 & 0.000 & 0.000 & 0.000 & 0.000 & 0.000 & 0.000 & 0.000 & 0.000 & 0.000 \\
\hline 1.75 & 1.319 & 1.421 & 1.286 & 1.319 & 1.231 & 1.187 & 1.123 & 1.120 & 1.032 & 0.970 & 0.922 & 0.870 & 0.772 & 0.717 \\
\hline 2.00 & 2.921 & 2.835 & 2.615 & 2.705 & 2.442 & 2.367 & 2.243 & 2.210 & 2.027 & 1.910 & 1.811 & 1.660 & 1.528 & 1.428 \\
\hline 2.25 & 4.634 & 4.393 & 4.162 & 4.102 & 3.737 & 3.548 & 3.343 & 3.220 & 2.997 & 2.850 & 2.660 & 2.440 & 2.247 & 2.140 \\
\hline 2.50 & 6.346 & 6.132 & 5.758 & 5.482 & 5.069 & 4.750 & 4.453 & 4.220 & 3.917 & 3.780 & 3.485 & 3.200 & 2.940 & 2.820 \\
\hline 2.75 & 8.187 & 7.872 & 7.358 & 6.842 & 6.398 & 5.960 & 5.543 & 5.220 & 4.837 & 4.700 & 4.280 & 3.940 & 3.622 & 3.470 \\
\hline 3.00 & 10.46 & 9.822 & 9.138 & 8.272 & 7.738 & 7.187 & 6.623 & 6.220 & 5.747 & 5.620 & 5.057 & 4.650 & 4.276 & 4.100 \\
\hline 3.25 & 12.72 & 12.05 & 11.06 & 9.992 & 9.088 & 8.409 & 7.713 & 7.170 & 6.627 & 6.480 & 5.797 & 5.340 & 4.926 & 4.710 \\
\hline 3.50 & 16.25 & 14.32 & 12.99 & 11.71 & 10.55 & 9.659 & 8.793 & 8.130 & 7.517 & 7.260 & 6.527 & 6.010 & 5.548 & 5.310 \\
\hline 3.75 & 21.22 & 17.04 & 15.39 & 13.50 & 12.15 & 10.98 & 9.913 & 9.100 & 8.407 & 8.060 & 7.257 & 6.670 & 6.169 & 5.890 \\
\hline 4.00 & 27.67 & 19.96 & 17.88 & 15.66 & 13.80 & 12.32 & 11.05 & 10.07 & 9.257 & 8.850 & 7.967 & 7.320 & 6.774 & 6.470 \\
\hline 4.25 & 34.30 & 23.75 & 20.45 & 17.95 & 15.47 & 13.69 & 12.21 & 11.06 & 10.13 & 9.650 & 8.677 & 7.970 & 7.369 & 7.040 \\
\hline 4.50 & 41.31 & 28.68 & 23.06 & 20.29 & 17.18 & 15.20 & 13.42 & 12.08 & 11.02 & 10.44 & 9.387 & 8.620 & 7.969 & 7.610 \\
\hline 4.75 & 48.32 & 34.46 & 27.56 & 22.96 & 19.69 & 16.79 & 14.77 & 13.13 & 11.97 & 11.24 & 10.11 & 9.280 & 8.579 & 8.160 \\
\hline 5.00 & 55.67 & 42.32 & 33.29 & 26.67 & 22.73 & 18.44 & 16.29 & 14.27 & 12.95 & 12.09 & 10.87 & 9.950 & 9.209 & 8.740 \\
\hline 5.25 & 65.40 & 50.31 & 39.71 & 32.68 & 25.92 & 21.12 & 17.85 & 15.78 & 14.08 & 12.99 & 11.67 & 10.66 & 9.899 & 9.340 \\
\hline 5.50 & 75.12 & 58.89 & 46.20 & 38.40 & 29.35 & 24.37 & 20.06 & 17.41 & 15.58 & 13.98 & 12.56 & 11.44 & 10.73 & 10.11 \\
\hline 5.75 & 87.91 & 68.64 & 52.69 & 43.44 & 33.25 & 27.64 & 23.20 & 19.35 & 17.32 & 15.21 & 13.65 & 12.51 & 11.60 & 10.99 \\
\hline 6.00 & 101.8 & 78.86 & 60.29 & 48.43 & 37.47 & 30.94 & 25.89 & 21.74 & 19.04 & 16.97 & 15.15 & 13.97 & 12.77 & 11.93 \\
\hline 6.25 & 117.5 & 90.97 & 68.44 & 54.61 & 41.95 & 33.84 & 28.14 & 23.89 & 20.85 & 18.59 & 16.54 & 14.98 & 13.83 & 12.90 \\
\hline 6.50 & 134.6 & 103.7 & 76.86 & 61.70 & 46.86 & 37.24 & 30.55 & 25.75 & 22.50 & 19.92 & 17.56 & 15.92 & 14.53 & 13.59 \\
\hline 6.75 & 152.9 & 117.3 & 87.51 & 69.39 & 52.11 & 41.14 & 33.37 & 27.75 & 24.15 & 21.28 & 18.61 & 16.71 & 15.21 & 14.14 \\
\hline 7.00 & 171.3 & 131.5 & 98.25 & 77.79 & 57.84 & 45.35 & 36.66 & 30.11 & 25.92 & 22.68 & 19.72 & 17.57 & 15.94 & 14.71 \\
\hline 7.25 & 190.6 & 146.1 & 109.6 & 86.81 & 64.22 & 49.91 & 40.23 & 32.84 & 27.97 & 24.18 & 20.91 & 18.58 & 16.74 & 15.34 \\
\hline 7.50 & 211.6 & 161.1 & 121.3 & 96.18 & 71.09 & 54.93 & 44.08 & 35.79 & 30.29 & 25.87 & 22.23 & 19.67 & 17.58 & 16.03 \\
\hline 7.75 & 234.6 & 177.5 & 133.6 & 105.6 & 78.50 & 60.32 & 48.19 & 38.77 & 32.77 & 27.76 & 23.70 & 20.82 & 18.49 & 16.75 \\
\hline 8.00 & 260.1 & 196.0 & 146.7 & 115.7 & 86.05 & 66.19 & 52.32 & 41.90 & 35.42 & 29.80 & 25.23 & 22.03 & 19.46 & 17.49 \\
\hline 8.25 & 288.9 & 217.2 & 160.5 & 127.1 & 94.01 & 72.60 & 56.72 & 45.29 & 38.05 & 31.91 & 26.86 & 23.28 & 20.44 & 18.30 \\
\hline 8.50 & 322.8 & 241.2 & 177.7 & 139.7 & 102.9 & 79.60 & 61.60 & 48.95 & 40.89 & 34.14 & 28.56 & 24.53 & 21.47 & 19.12 \\
\hline 8.75 & 363.7 & 268.6 & 197.2 & 153.9 & 113.1 & 87.22 & 66.89 & 53.02 & 44.02 & 36.54 & 30.38 & 25.89 & 22.58 & 19.97 \\
\hline 9.00 & 411.1 & 301.4 & 219.4 & 171.6 & 124.3 & 95.52 & 73.07 & 57.43 & 47.44 & 39.14 & 32.31 & 27.46 & 23.81 & 20.94 \\
\hline 9.25 & 457.6 & 339.0 & 246.2 & 191.7 & 138.9 & 104.5 & 80.58 & 62.78 & 51.34 & 42.10 & 34.43 & 29.22 & 25.19 & 22.04 \\
\hline 9.50 & 508.2 & 377.0 & 276.1 & 214.1 & 155.7 & 117.0 & 89.01 & 69.27 & 56.25 & 45.37 & 37.10 & 31.32 & 26.89 & 23.32 \\
\hline 9.75 & 547.2 & 412.0 & 307.1 & 239.8 & 173.7 & 131.4 & 100.0 & 76.87 & 62.24 & 49.59 & 40.33 & 33.85 & 28.93 & 24.79 \\
\hline 10.00 & 589.5 & 446.5 & 335.6 & 267.7 & 193.2 & 146.7 & 112.5 & 86.46 & 69.49 & 54.71 & 44.21 & 37.08 & 31.56 & 26.67 \\
\hline 10.25 & 635.0 & 479.3 & 362.2 & 292.3 & 215.6 & 163.5 & 124.7 & 97.02 & 78.11 & 60.81 & 48.97 & 40.76 & 34.69 & 29.01 \\
\hline 10.50 & 673.6 & 512.3 & 386.2 & 313.5 & 234.6 & 180.4 & 138.3 & 107.1 & 86.93 & 68.04 & 54.70 & 45.39 & 38.36 & 31.74 \\
\hline 10.75 & 715.8 & 541.6 & 412.6 & 335.6 & 253.3 & 195.2 & 153.4 & 118.5 & 95.91 & 75.64 & 60.98 & 50.50 & 42.51 & 34.85 \\
\hline 11.00 & 762.2 & 571.6 & 440.2 & 355.9 & 269.9 & 209.3 & 165.7 & 130.3 & 105.7 & 83.14 & 66.78 & 55.83 & 46.53 & 38.24 \\
\hline 11.25 & 813.6 & 603.7 & 464.6 & 377.3 & 284.6 & 223.5 & 177.7 & 140.4 & 114.3 & 91.54 & 73.13 & 60.75 & 50.34 & 41.48 \\
\hline 11.50 & 869.2 & 637.0 & 489.4 & 396.0 & 300.8 & 237.0 & 187.3 & 149.9 & 121.1 & 99.24 & 79.95 & 65.55 & 54.40 & 44.25 \\
\hline 11.75 & 920.8 & 673.7 & 516.6 & 415.2 & 317.0 & 249.3 & 197.7 & 158.9 & 128.5 & 105.0 & 84.82 & 70.35 & 58.73 & 47.19 \\
\hline 12.00 & 975.4 & 713.8 & 546.4 & 436.2 & 332.6 & 261.8 & 209.0 & 167.0 & 136.5 & 111.3 & 89.90 & 74.95 & 62.18 & 50.27 \\
\hline 12.25 & 1034. & 756.3 & 578.9 & 459.2 & 349.7 & 274.1 & 219.3 & 175.8 & 145.2 & 118.1 & 95.36 & 79.25 & 65.64 & 53.55 \\
\hline 12.50 & 1095. & 801.4 & 613.5 & 484.3 & 368.4 & 287.4 & 229.3 & 184.0 & 154.3 & 125.4 & 101.1 & 83.80 & 69.32 & 56.94 \\
\hline 12.75 & 1160. & 849.1 & 650.2 & 513.2 & 388.7 & 302.1 & 240.2 & 192.7 & 161.7 & 133.1 & 107.3 & 88.40 & 73.16 & 60.21 \\
\hline 13.00 & 1229. & 899.8 & 689.0 & 544.1 & 411.5 & 317.9 & 252.1 & 202.1 & 169.7 & 139.7 & 113.6 & 93.20 & 77.25 & 63.71 \\
\hline 13.25 & 1302. & 953.3 & 730.1 & 576.7 & 436.1 & 336.6 & 265.4 & 212.3 & 178.3 & 146.4 & 120.7 & 98.30 & 81.99 & 67.40 \\
\hline 13.50 & 1379. & 1011. & 773.7 & 611.3 & 462.2 & 356.7 & 281.6 & 224.3 & 187.5 & 153.8 & 128.1 & 103.8 & 87.01 & 71.28 \\
\hline 13.75 & 1461. & 1071. & 819.8 & 647.9 & 489.8 & 377.9 & 298.9 & 238.4 & 199.1 & 161.6 & 136.0 & 109.6 & 92.33 & 75.31 \\
\hline 14.00 & 1547. & 1134. & 868.7 & 686.7 & 519.0 & 400.5 & 317.1 & 253.2 & 211.4 & 173.1 & 144.4 & 116.8 & 97.94 & 80.17 \\
\hline 14.25 & 1639. & 1202. & 920.5 & 727.8 & 550.0 & 424.3 & 336.5 & 269.0 & 224.4 & 185.5 & 153.2 & 124.8 & 103.9 & 85.41 \\
\hline 14.50 & 1736. & 1273. & 975.3 & 771.3 & 582.9 & 449.6 & 357.0 & 285.7 & 238.2 & 198.6 & 162.6 & 133.3 & 110.2 & 90.96 \\
\hline 14.75 & 1839. & 1349. & 1034. & 817.4 & 617.7 & 476.4 & 378.6 & 303.4 & 252.9 & 212.4 & 172.6 & 142.3 & 116.9 & 96.87 \\
\hline 15.00 & 1948. & 1429. & 1095. & 866.2 & 654.5 & 504.8 & 401.6 & 322.1 & 268.3 & 227.1 & 183.1 & 151.8 & 124.0 & 103.1 \\
\hline X-ray ${ }^{b}$ & 3850 & 2700 & 2200 & 1700 & 1150 & 870 & 620 & 440 & 326 & 240 & 162 & 109 & 74.1 & 48.7 \\
\hline$m_{\mathrm{w}}^{\prime c}$ & 17.6 & 17.3 & 17.0 & 16.8 & 16.6 & 16.3 & 16.2 & 16.1 & 15.9 & 15.9 & 15.7 & 15.7 & 15.8 & 15.7 \\
\hline $\log f_{\mathrm{s}}{ }^{\mathrm{d}}$ & 0.81 & 0.72 & 0.62 & 0.52 & 0.42 & 0.31 & 0.21 & 0.10 & 0.01 & -0.07 & -0.16 & -0.27 & -0.36 & -0.46 \\
\hline$M_{\mathrm{w}}{ }^{\mathrm{e}}$ & 5.3 & 4.8 & 4.3 & 3.8 & 3.3 & 2.8 & 2.4 & 2.0 & 1.6 & 1.4 & 1.0 & 0.8 & 0.6 & 0.2 \\
\hline
\end{tabular}

a chemical composition of the envelope is assumed to be that of "CO nova 2" in Table 1

${ }^{b}$ duration of supersoft $\mathrm{X}$-ray phase in units of days

c converted magnitudes at the bottom point in Figure 2

$\mathrm{d}$ stretching factors against V1668 Cyg observational data in Figure 2

e absolute magnitudes at the bottom point in Figure 2 by assuming $(m-M)_{V}=14.3(\mathrm{~V} 1668 \mathrm{Cyg})$ 
TABLE 3

Light CuRves of Ne NovaE ${ }^{\mathrm{a}}$

\begin{tabular}{|c|c|c|c|c|c|c|c|c|c|c|c|c|c|}
\hline $\begin{array}{c}m_{\mathrm{ff}} \\
(\mathrm{mag})\end{array}$ & $\begin{array}{c}0.7 M_{\odot} \\
\text { (day) }\end{array}$ & $\begin{array}{c}0.75 M_{\odot} \\
\quad \text { (day) }\end{array}$ & $\begin{array}{c}0.8 M_{\odot} \\
\text { (day) }\end{array}$ & $\begin{array}{c}0.85 M_{\odot} \\
\quad \text { (day) }\end{array}$ & $\begin{array}{c}0.9 M_{\odot} \\
\text { (day) }\end{array}$ & $\begin{array}{c}0.95 M_{\odot} \\
\text { (day) }\end{array}$ & $\begin{array}{c}1.0 M_{\odot} \\
\text { (day) }\end{array}$ & $\begin{array}{c}1.05 M_{\odot} \\
\text { (day) }\end{array}$ & $\begin{array}{c}1.1 M_{\odot} \\
\text { (day) }\end{array}$ & $\begin{array}{c}1.15 M_{\odot} \\
\text { (day) }\end{array}$ & $\begin{array}{c}1.2 M_{\odot} \\
\text { (day) }\end{array}$ & $\begin{array}{c}1.25 M_{\odot} \\
\text { (day) }\end{array}$ & $\begin{array}{c}1.3 M_{\odot} \\
\text { (day) }\end{array}$ \\
\hline 3.000 & 0.0 & 0.0 & 0.0 & 0.0 & 0.0 & 0.0 & 0.0 & 0.0 & 0.0 & 0.0 & 0.0 & 0.0 & 0.0 \\
\hline 3.250 & 2.830 & 2.270 & 1.610 & 1.292 & 1.120 & 0.960 & 0.870 & 0.758 & 0.667 & 0.608 & 0.565 & 0.515 & 0.471 \\
\hline 3.500 & 6.860 & 4.590 & 3.230 & 2.742 & 2.270 & 1.940 & 1.740 & 1.499 & 1.352 & 1.215 & 1.117 & 1.026 & 0.939 \\
\hline 3.750 & 10.98 & 6.970 & 5.070 & 4.292 & 3.450 & 2.960 & 2.630 & 2.259 & 2.005 & 1.828 & 1.669 & 1.535 & 1.408 \\
\hline 4.000 & 15.27 & 9.400 & 7.630 & 5.882 & 4.770 & 4.020 & 3.560 & 3.035 & 2.683 & 2.429 & 2.227 & 2.039 & 1.872 \\
\hline 4.250 & 19.66 & 12.35 & 10.34 & 7.572 & 6.260 & 5.150 & 4.500 & 3.874 & 3.372 & 3.052 & 2.784 & 2.543 & 2.336 \\
\hline 4.500 & 24.59 & 16.54 & 13.12 & 9.872 & 7.810 & 6.460 & 5.480 & 4.736 & 4.154 & 3.716 & 3.429 & 3.114 & 2.863 \\
\hline 4.750 & 30.87 & 20.97 & 16.18 & 12.51 & 9.470 & 8.050 & 6.870 & 5.736 & 4.981 & 4.466 & 4.097 & 3.717 & 3.480 \\
\hline 5.000 & 38.11 & 25.58 & 19.56 & 15.25 & 11.51 & 9.680 & 8.330 & 6.980 & 5.865 & 5.276 & 4.817 & 4.346 & 4.068 \\
\hline 5.250 & 45.78 & 31.11 & 23.10 & 17.98 & 13.69 & 11.34 & 9.740 & 8.270 & 6.945 & 6.126 & 5.557 & 4.996 & 4.600 \\
\hline 5.500 & 54.00 & 37.61 & 26.89 & 20.83 & 15.97 & 13.10 & 11.05 & 9.340 & 7.935 & 7.046 & 6.297 & 5.616 & 5.119 \\
\hline 5.750 & 65.14 & 44.69 & 32.20 & 23.82 & 18.41 & 15.02 & 12.46 & 10.38 & 8.855 & 7.846 & 6.997 & 6.186 & 5.588 \\
\hline 6.000 & 78.08 & 52.44 & 38.16 & 27.74 & 21.03 & 17.16 & 14.13 & 11.57 & 9.745 & 8.566 & 7.647 & 6.756 & 6.043 \\
\hline 6.250 & 92.06 & 62.15 & 44.65 & 32.40 & 24.08 & 19.48 & 16.08 & 13.08 & 10.83 & 9.416 & 8.327 & 7.326 & 6.502 \\
\hline 6.500 & 106.9 & 72.79 & 51.93 & 37.53 & 27.75 & 22.27 & 18.22 & 14.77 & 12.09 & 10.37 & 9.087 & 7.886 & 6.973 \\
\hline 6.750 & 121.6 & 84.36 & 59.93 & 43.19 & 31.76 & 25.48 & 20.73 & 16.64 & 13.50 & 11.46 & 9.937 & 8.546 & 7.467 \\
\hline 7.000 & 137.5 & 95.87 & 68.63 & 49.46 & 36.14 & 28.98 & 23.51 & 18.75 & 15.04 & 12.65 & 10.86 & 9.206 & 7.997 \\
\hline 7.250 & 153.6 & 107.6 & 77.53 & 56.30 & 40.95 & 32.66 & 26.49 & 21.09 & 16.78 & 13.95 & 11.87 & 9.956 & 8.537 \\
\hline 7.500 & 171.0 & 120.3 & 86.47 & 63.47 & 46.23 & 36.67 & 29.55 & 23.51 & 18.58 & 15.37 & 12.98 & 10.72 & 9.087 \\
\hline 7.750 & 190.3 & 134.1 & 96.17 & 70.30 & 51.94 & 41.04 & 32.87 & 25.93 & 20.38 & 16.77 & 14.09 & 11.53 & 9.627 \\
\hline 8.000 & 214.2 & 149.1 & 107.7 & 77.72 & 57.59 & 45.66 & 36.49 & 28.49 & 22.32 & 18.22 & 15.23 & 12.35 & 10.19 \\
\hline 8.250 & 241.0 & 167.0 & 120.4 & 87.02 & 63.69 & 50.42 & 40.36 & 31.26 & 24.43 & 19.81 & 16.44 & 13.18 & 10.77 \\
\hline 8.500 & 270.1 & 187.9 & 134.9 & 97.51 & 71.21 & 55.63 & 44.52 & 34.40 & 26.85 & 21.55 & 17.74 & 14.12 & 11.41 \\
\hline 8.750 & 301.9 & 211.4 & 151.8 & 109.3 & 79.80 & 62.42 & 49.45 & 38.30 & 29.64 & 23.69 & 19.26 & 15.25 & 12.18 \\
\hline 9.000 & 338.4 & 235.7 & 170.6 & 122.9 & 89.49 & 69.91 & 55.54 & 42.96 & 32.86 & 26.07 & 21.07 & 16.60 & 13.15 \\
\hline 9.250 & 376.5 & 263.7 & 191.0 & 138.2 & 100.7 & 78.89 & 62.33 & 48.14 & 36.75 & 29.04 & 23.32 & 18.17 & 14.32 \\
\hline 9.500 & 409.3 & 295.3 & 214.2 & 155.4 & 113.3 & 89.13 & 70.38 & 54.26 & 41.14 & 32.40 & 25.95 & 20.02 & 15.61 \\
\hline 9.750 & 447.0 & 320.0 & 240.5 & 175.0 & 127.4 & 100.4 & 79.33 & 61.20 & 46.37 & 36.42 & 28.95 & 22.15 & 17.15 \\
\hline 10.00 & 490.2 & 347.6 & 259.3 & 196.8 & 143.3 & 113.2 & 89.49 & 69.13 & 52.20 & 40.94 & 32.36 & 24.74 & 19.00 \\
\hline 10.25 & 539.6 & 379.0 & 280.5 & 211.7 & 161.0 & 127.6 & 100.9 & 78.49 & 59.10 & 46.07 & 36.50 & 27.61 & 21.04 \\
\hline 10.50 & 577.7 & 414.4 & 304.3 & 228.4 & 173.3 & 140.5 & 113.9 & 87.86 & 66.88 & 51.98 & 41.46 & 30.91 & 23.52 \\
\hline 10.75 & 612.7 & 448.9 & 331.2 & 247.1 & 186.9 & 151.2 & 123.1 & 97.18 & 74.73 & 58.50 & 46.47 & 34.56 & 26.20 \\
\hline 11.00 & 651.7 & 474.6 & 358.2 & 267.9 & 202.1 & 162.9 & 132.6 & 106.4 & 82.36 & 64.22 & 51.36 & 38.45 & 28.83 \\
\hline 11.25 & 694.6 & 502.9 & 378.2 & 289.6 & 218.9 & 175.9 & 143.1 & 115.0 & 89.13 & 69.31 & 56.29 & 41.87 & 31.37 \\
\hline 11.50 & 741.9 & 534.3 & 400.3 & 305.4 & 236.6 & 190.1 & 154.6 & 122.7 & 95.41 & 74.79 & 60.80 & 45.47 & 33.74 \\
\hline 11.75 & 787.7 & 568.8 & 424.7 & 322.8 & 249.5 & 203.3 & 167.1 & 131.0 & 102.1 & 80.68 & 65.21 & 49.08 & 36.17 \\
\hline 12.00 & 835.3 & 605.2 & 451.6 & 342.0 & 263.7 & 214.6 & 177.0 & 139.3 & 109.3 & 86.96 & 69.69 & 52.43 & 38.69 \\
\hline 12.25 & 885.6 & 641.6 & 479.8 & 363.0 & 279.2 & 227.0 & 186.9 & 147.6 & 116.1 & 93.09 & 74.47 & 55.96 & 41.36 \\
\hline 12.50 & 939.0 & 680.3 & 509.2 & 385.4 & 296.3 & 240.7 & 197.7 & 156.7 & 123.4 & 98.90 & 79.37 & 59.84 & 44.29 \\
\hline 12.75 & 995.4 & 721.2 & 540.4 & 408.7 & 314.7 & 255.4 & 209.5 & 166.6 & 131.2 & 105.2 & 84.67 & 63.97 & 47.35 \\
\hline 13.00 & 1055. & 764.5 & 573.4 & 433.5 & 334.1 & 271.2 & 222.4 & 177.1 & 139.6 & 112.1 & 90.29 & 68.34 & 50.60 \\
\hline 13.25 & 1118 & 810.4 & 608.4 & 459.7 & 354.7 & 287.9 & 236.2 & 187.8 & 148.3 & 119.2 & 96.26 & 72.90 & 53.86 \\
\hline 13.50 & 1185. & 859.0 & 645.4 & 487.5 & 376.5 & 305.7 & 250.9 & 199.2 & 157.4 & 126.6 & 102.3 & 77.56 & 57.21 \\
\hline 13.75 & 1257. & 910.6 & 684.6 & 517.0 & 399.6 & 324.4 & 266.4 & 211.2 & 167.0 & 134.5 & 108.8 & 82.50 & 60.77 \\
\hline 14.00 & 1332. & 965.1 & 726.2 & 548.2 & 424.0 & 344.4 & 282.9 & 223.9 & 177.2 & 142.9 & 115.6 & 87.73 & 64.53 \\
\hline 14.25 & 1412. & 1023. & 770.2 & 581.2 & 450.0 & 365.4 & 300.4 & 237.4 & 188.0 & 151.7 & 122.9 & 93.27 & 68.52 \\
\hline 14.50 & 1496. & 1084. & 816.8 & 616.2 & 477.4 & 387.7 & 318.8 & 251.7 & 199.4 & 161.1 & 130.5 & 99.16 & 72.75 \\
\hline 14.75 & 1586. & 1149. & 866.2 & 653.2 & 506.5 & 411.4 & 338.4 & 266.8 & 211.5 & 171.1 & 138.6 & 105.4 & 77.22 \\
\hline 15.00 & 1681. & 1218. & 918.5 & 692.5 & 537.2 & 436.4 & 359.1 & 282.9 & 224.3 & 181.6 & 147.2 & 112.0 & 81.96 \\
\hline X-ray ${ }^{b}$ & 4300 & 3100 & 2240 & 1620 & 1170 & 864 & 640 & 440 & 300 & 201 & 135 & 79.3 & 42.2 \\
\hline$m_{\mathrm{w}}^{\prime c}$ & 15.2 & 14.9 & 14.8 & 14.5 & 14.3 & 14.2 & 14.0 & 14.0 & 13.8 & 13.8 & 13.8 & 13.8 & 13.8 \\
\hline $\log f_{\mathrm{s}}^{\mathrm{d}}$ & 0.72 & 0.60 & 0.48 & 0.38 & 0.27 & 0.18 & 0.09 & -0.02 & -0.12 & -0.22 & -0.31 & -0.44 & -0.60 \\
\hline$M_{\mathrm{W}}{ }^{\mathrm{e}}$ & 4.7 & 4.1 & 3.7 & 3.1 & 2.7 & 2.3 & 2.0 & 1.6 & 1.2 & 0.9 & 0.7 & 0.4 & 0.0 \\
\hline
\end{tabular}

a chemical composition of the envelope is assumed to be that of "Ne nova 2" in Table 1

$\mathrm{b}$ duration of supersoft X-ray phase in units of days

c converted magnitudes at the bottom point in Figure 6

d stretching factor against V1974 Cyg observational data in Figure 6

$\mathrm{e}$ absolute magnitudes at the bottom point in Figure 6 by assuming $(m-M)_{V}=12.3(\mathrm{~V} 1974 \mathrm{Cyg})$ 


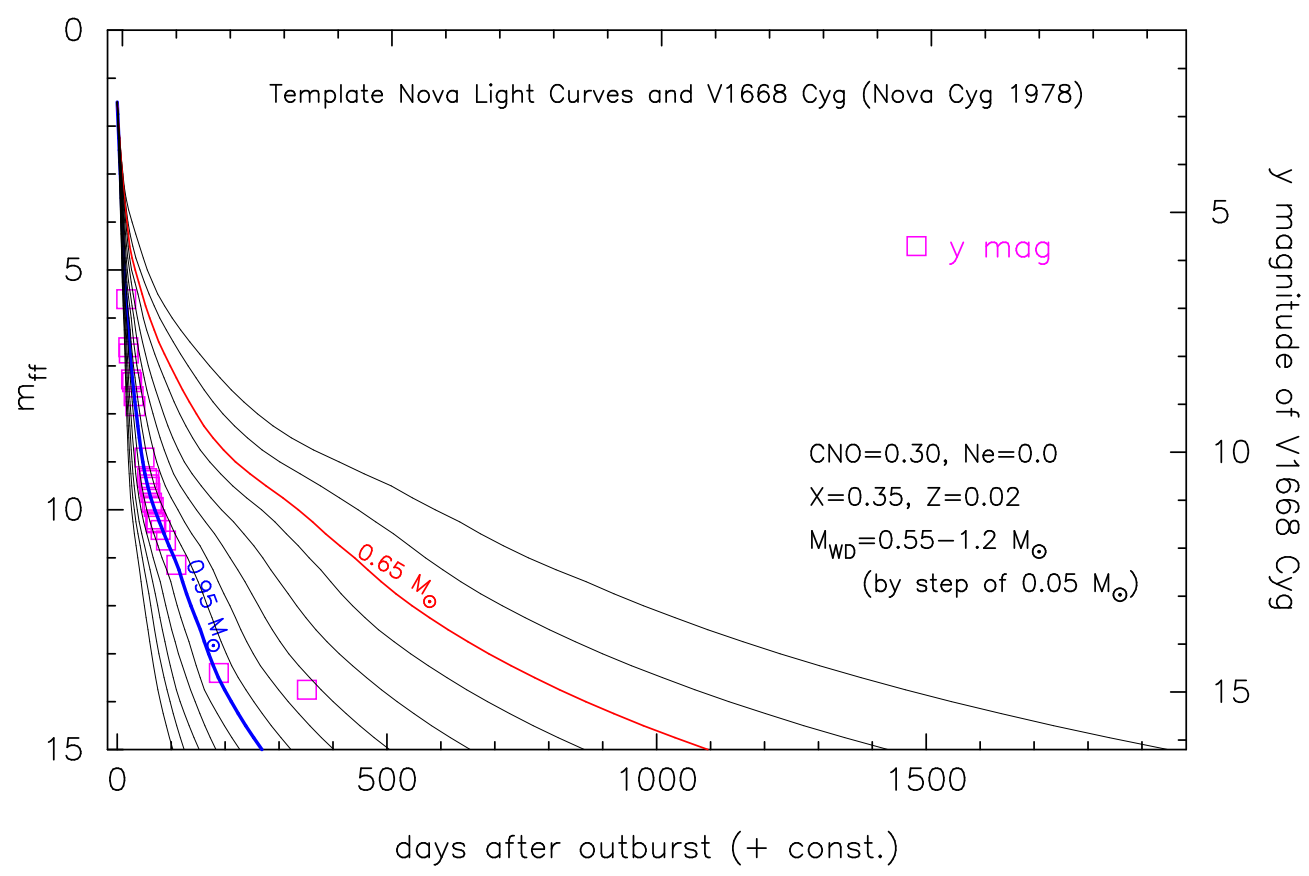

FIG. 1.- Magnitudes of our free-free emission model light curves for various WD masses, i.e., $0.55-1.2 M_{\odot}$ by $0.05 M_{\odot}$ step, numerical data of which are tabulated in Table 2 We adopt a chemical composition of "CO nova 2" in Table 1 for these WD envelopes. The decay timescale depends mainly on the WD mass. We add observational y magnitudes (open squares) of V1668 Cyg (Nova Cygni 1978) for comparison, which are taken from Gallagher et al. (1980). The $y$ magnitudes are shifted up by $1.2 \mathrm{mag}$ to fit them with the $0.95 M_{\odot}$ WD model (see the right axis). Our light curve model of $0.95 M_{\odot}$ WD (blue color line) almost perfectly fits with the observational $y$ magnitudes except the last point.

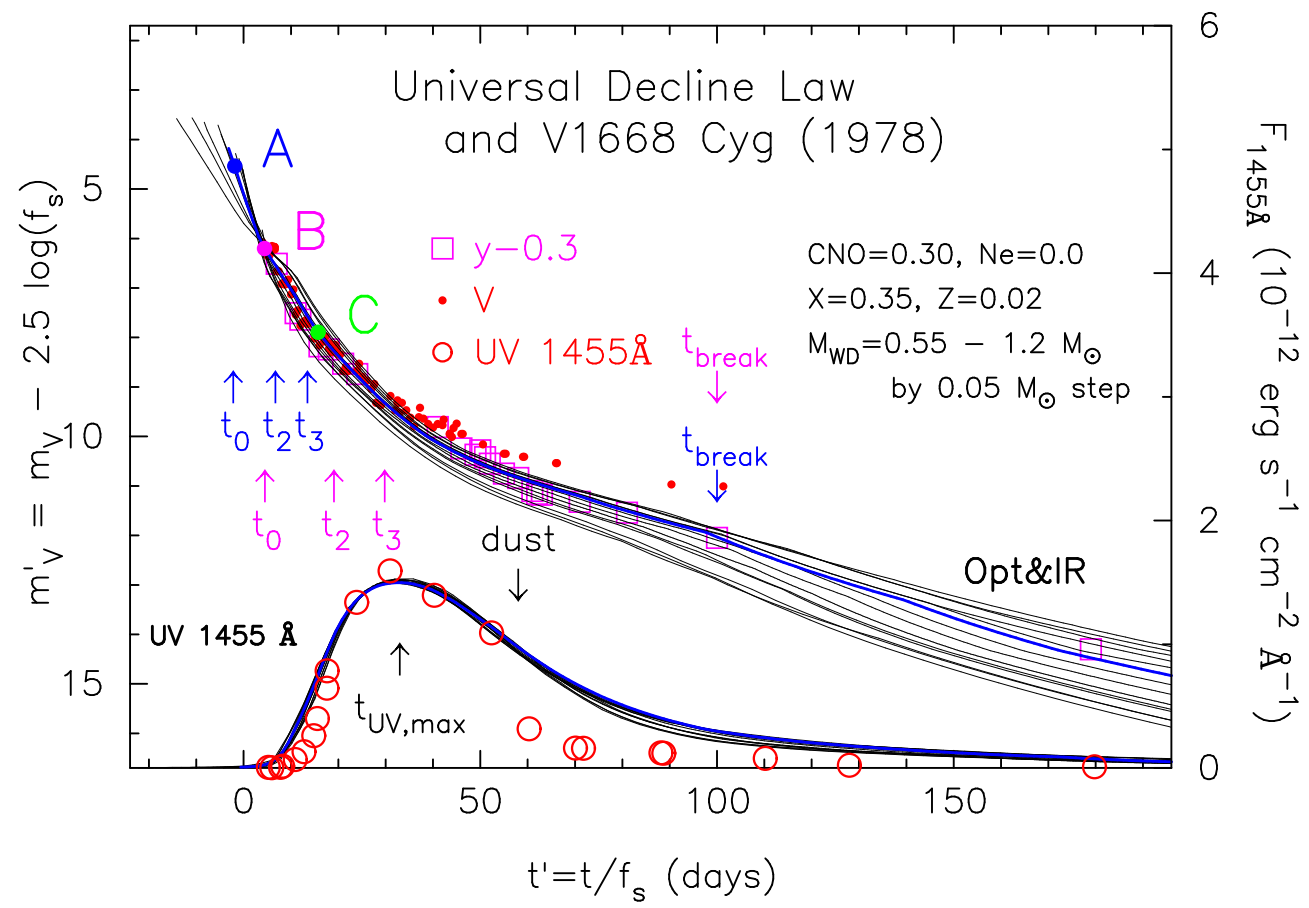

FIG. 2. - Same as Figure 1 but in the plane of rescaled flux, $-2.5 \log f_{\mathrm{s}} F_{\nu}+$ const. $=m_{V}-2.5 \log f_{\mathrm{s}}=m_{V}^{\prime}$ and scaled time, $t / f_{\mathrm{s}}=t^{\prime}$. We added UV $1455 \AA$ model light curves which are stretched along time by a factor of $1 / f_{\mathrm{s}}$. This stretching factor is determined in a way that each UV light curve is overlapped to that of V1668 Cyg observation (red big open circles), data of which are taken from Cassatella et al. (2002). The UV model fluxes are normalized to the peak value of the UV observation. The values of $\log f_{\mathrm{s}}$ are tabulated in Table 2 The sudden drop in the UV flux of V1668 Cyg on day $\sim 60$ is caused by formation of an optically thin dust shell (denoted by an arrow with "dust"). All the free-free emission model light curves are almost overlapped to each other. We call this homologous nature of free-free light curves "the universal decline law." Here $t_{2}$ and $t_{3}$ times are not uniquely determined along our universal decline law, because these times depend on the initial envelope mass as indicated by two sets of $\left(t_{0}, t_{2}, t_{3}\right)$ in the figure. Three different initial envelope masses of $M_{\text {env }, 0}=2.3 \times 10^{-5}$, $1.6 \times 10^{-5}$, and $1.1 \times 10^{-5} M_{\odot}$ correspond to three peak brightnesses, points A, B, and C, respectively, on the $0.95 M_{\odot}$ WD model. We added the $V$ magnitude observation (red filled circles) for comparison, data of which are taken from Mallama \& Skillman (1979). The $y$ magnitudes are shifted up by 0.3 mag to match them with the $V$ magnitudes in the early phase. 
These light curves demonstrate that the decay timescales of novae are sensitive to the WD mass. Therefore we can determine the WD mass of a nova from light curve fitting. The $0.95 M_{\odot}$ (blue thick solid line) or $1.0 M_{\odot}$ WD may be a typical case of fast novae and the light curve of $0.65 M_{\odot}$ WD (red thick solid line) may correspond to a slow nova.

These free-free emission model light curves are applicable to optical and near infrared (IR) wavelength regions (e.g., Hachisu \& Kato 2006). We added observational y magnitudes of V1668 Cyg (Nova Cygni 1978) to Figure 1, which nicely reproduce the light curve of $0.95 M_{\odot}$ WD. In this case, we shift the model light curve of $0.95 M_{\odot}$ WD down by 1.2 mag in order to fit it with the observation (see the right axis of Figure 11). The intermediate width $y$ band is an emission line-free optical band, so it is ideal to fit our model light curve with $y$ magnitudes.

In this way, we can shift the template light curves to fit them with observation for individual novae. In other words, one can shift the model light curve "up and down" against the observational data of an individual nova. In the case of V1668 Cyg, we obtain $m_{y}=m_{\mathrm{ff}}+1.2$ from the $0.95 M_{\odot}$ WD model in Table 2

\subsection{Brightness of free-free light curves}

The free-free emission model light curves in Figure 1 have strong similarity in their shapes. In our previous paper (Hachisu \& Kato 2006), we showed that these model light curves are homologous and their shapes are almost overlapped with each other when they are properly squeezed or stretched along time. In this subsection, we reformulate this homologous properties in a more sophisticated manner and obtain their brightnesses.

First, we squeeze or stretch our UV $1455 \AA$ model light curves in the direction of time and also its peak flux is normalized to be fitted with the observational peak of V1668 Cyg. Then we obtain all the UV light curves almost overlapped to each other as shown in Figure 2 . We determine a stretching factor, $f_{\mathrm{s}}$, of each UV model light curve by increasing or decreasing it until the model light curve shape matches the observation. Table 2 shows $\log$ arithmic values of $\log f_{\mathrm{s}}$, that is, positive (negative) for stretching (squeezing) against the V1668 Cyg UV $1455 \AA$ observation. For example, we see that the $0.95 M_{\odot}$ WD model light curve is almost overlapped to the V1668 Cyg observation without stretching $\left(f_{\mathrm{s}} \approx 1.0\right)$ and the $0.8 M_{\odot}$ WD model light curve evolves about twice slower $\left(f_{\mathrm{s}} \approx 2.0\right)$ than the $0.95 M_{\odot}$ WD model. In this way, all the UV $1455 \AA$ model light curves are overlapped to each other, if we squeeze/stretch the time as $t^{\prime}=t / f_{\mathrm{s}}$. Note that the stretching factor is slightly different from that in our previous paper (Table 9 of Hachisu \& Kato 2006), which was determined in the way that each free-free model light curve is overlapped with each other instead of UV $1455 \AA$ model light curves.

Figure 2 also shows optical data ( $y$ and $V$ magnitudes) of V1668 Cyg and fitted free-free emission model light curve of $0.95 M_{\odot}$ WD $\left(f_{\mathrm{s}} \approx 1.0\right)$. The other free-free emission model light curves are squeezed/stretched with the same $f_{\mathrm{s}}$ as that of UV light curves. When we squeeze the model light curves by a factor of $f_{\mathrm{s}}$ in the time direction $\left(t^{\prime}=t / f_{\mathrm{s}}\right.$ and $\left.\nu^{\prime}=f_{\mathrm{s}} \nu\right)$, the flux is also changed as $F_{\nu^{\prime}}^{\prime}=f_{\mathrm{s}} F_{\nu}$ because

$$
\frac{d}{d t^{\prime}}=f_{\mathrm{s}} \frac{d}{d t} \text {. }
$$

Therefore, we shift the model light curves in the vertical direction as

$$
m_{V}^{\prime}=m_{V}-2.5 \log f_{\mathrm{s}}
$$

and obtained Figure 2. Here $m_{V}$ is the apparent $V$ magnitude of an $M_{\mathrm{WD}}$ model and $m_{V}^{\prime}$ is its time-stretched magnitude against a standard model of $0.95 M_{\odot}$ WD $(\approx \mathrm{V} 1668 \mathrm{Cyg})$. We see that all the light curves are almost overlapped to each other. For more details of derivation of Equation (5), see Appendix A.

The origins of magnitude in Figures 1 and 2 are different because the magnitude in the right axis of Figure 1 is calibrated by the $y$ magnitudes whereas by the $V$ magnitudes in Figure 2] We define the rescaled apparent $V$ magnitude (timestretched $V$ magnitude) against the $V$ magnitudes of V1668 Cyg instead of the $y$ magnitudes. The apparent $V$ magnitudes are written as

$$
m_{V}^{\prime}=-2.5 \log \left[\frac{\dot{M}_{\mathrm{wind}}^{2}}{v_{\mathrm{ph}}^{2} R_{\mathrm{ph}}}\right]_{\left(t / f_{\mathrm{s}}\right)}^{\left\{M_{\mathrm{wD}}\right\}}+K_{V},
$$

where $K_{V}$ is a constant and is determined to match $m_{V}^{\prime}$ of the $0.95 M_{\odot}$ WD model with the $V$ magnitude of V1668 Cyg as shown in Figure 2. Therefore, $K_{V}$ is also related to $G^{\left\{0.95 M_{\odot}\right\}}$ as

$$
K_{V}=G^{\left\{0.95 M_{\odot}\right\}}+0.9 .
$$

This simply means

$$
m_{V}^{\prime}=m_{V}=m_{\mathrm{ff}}+0.9,
$$

for $0.95 M_{\odot}$ WD model with $f_{\mathrm{s}}=1.0$. Note that we here obtain 0.9 instead of 1.2 because observational $m_{V}$ is brighter than $m_{y}$ by about $0.3 \mathrm{mag}$, that is, $m_{V}$ and $m_{y}-0.3$ are overlapped to each other in Figure 2. We use the same $K_{V}$ for all other WD mass models (from $M_{\mathrm{WD}}=0.55$ to $1.2 M_{\odot}$ by 0.05 $M_{\odot}$ step) in Figure 2. All the free-free emission model light curves are almost overlapped to each other.

It should be noticed here that the flux is condensed and increased by a factor of $f_{\mathrm{s}}$ when the time is stretched by a factor of $1 / f_{\mathrm{s}}$ (or squeezed by a factor of $f_{\mathrm{s}}$ ) only under the condition that the spectrum is not changed after time-stretching. This condition is satisfied in free-free emission (optical and near IR) because the flux ( $F_{\nu} \sim$ constant $)$ is independent of the frequency $\nu$, but not satisfied in blackbody emission because the blackbody emissivity depends on the frequency $\nu$ and, after time-stretching, a blackbody spectrum changes with $\nu^{\prime}=f_{\mathrm{s}} \nu$. See Appendix A for more detailed formulation of the scaling of free-free flux.

\subsection{Maximum brightness vs. initial envelope mass}

Figure 2 shows three points of A, B, and C on the same light curve of $0.95 M_{\odot}$ WD model. Point B indicates the initial envelope mass of $M_{\mathrm{env}, 0}=1.6 \times 10^{-5} M_{\odot}$, corresponding to $t_{0}$ at the maximum brightness of V1668 Cyg. Point A indicates a much brighter maximum and corresponds to a much larger envelope mass $\left(M_{\mathrm{env}, 0}=2.3 \times 10^{-5} M_{\odot}\right)$ than that of point $\mathrm{B}$. On the other hand, point $\mathrm{C}$ corresponds to a much smaller envelope mass $\left(M_{\mathrm{env}, 0}=1.1 \times 10^{-5} M_{\odot}\right)$.

The initial envelope mass depends on the mass accretion rate to the WD before ignition (see, e.g., Figure 9 of Nomoto 1982; Prialnik \& Kovetz 1995). Point A is corresponding to a case of much lower mass accretion rates to the WD. On the other hand, point $\mathrm{C}$ is corresponding to a case of much 


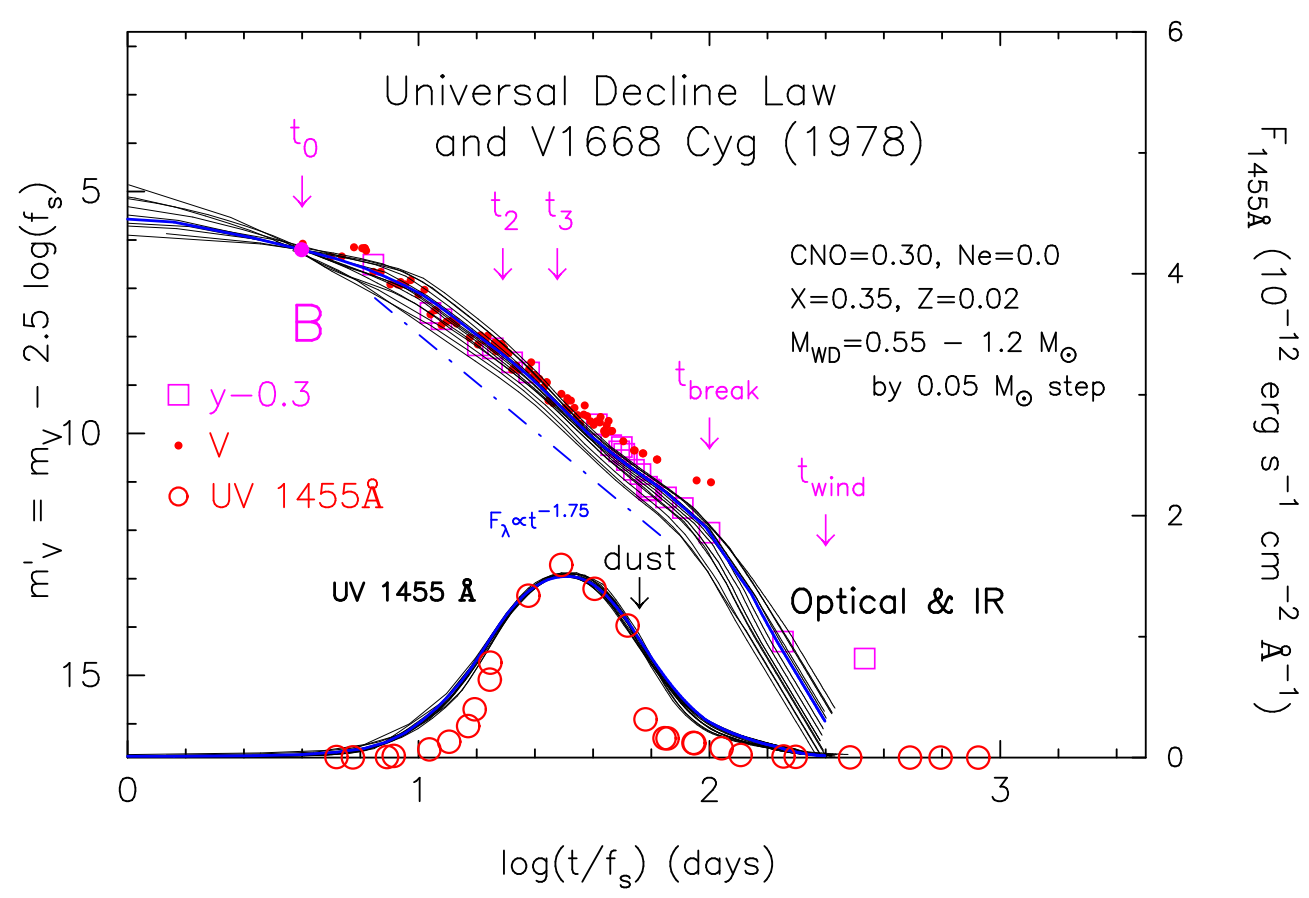

FIG. 3.- Same as Figure 2 but in the logarithmic time vs. magnitude plane. The origin of time is about 4 days before the optical maximum of V1668 Cyg. The right edges of free-free emission model light curves correspond to the epoch when the optically thick winds stop (denote by an arrow labeled $t_{\text {wind }}$ ). We also added a decline rate of free-free flux, i.e., $F_{\lambda} \propto t^{-1.75}$ (blue dash-dotted line) in the early phase before $t_{\text {break }}$.

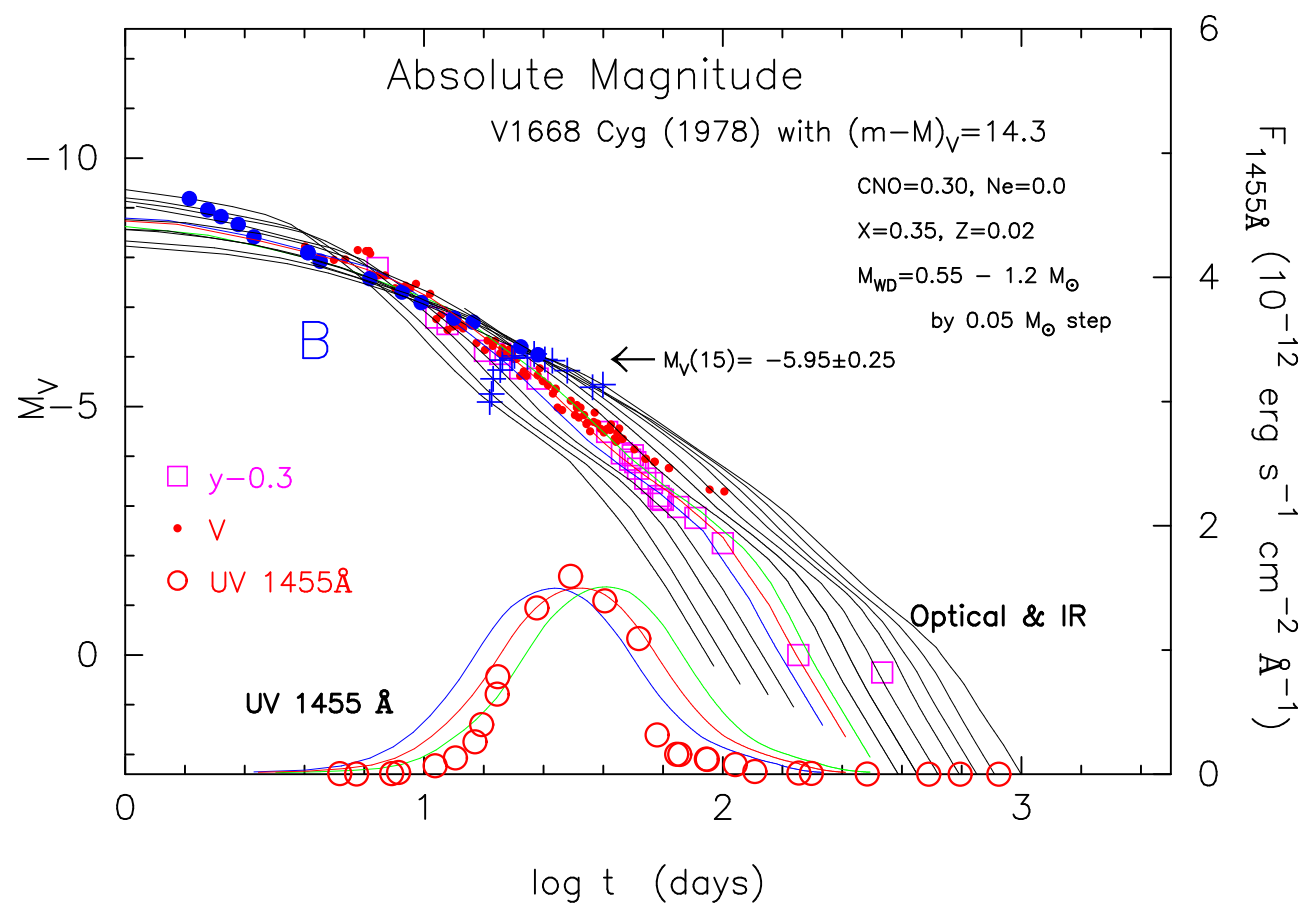

FIG. 4.- Same as Figure 3 but for absolute magnitudes and real timescales. We have calibrated the free-free model light curves by a distance modulus of $(m-M)_{V}=14.3$ for V1668 Cyg. The position at point B in Figure 3 is indicated by a blue filled circle. We also show the magnitude, $M_{V}(15)$, at 15 days after the optical maximum by a blue cross. Their average value of $M_{V}(15)=-5.95 \pm 0.25$ is also indicated in the figure for $0.7-1.05 M_{\odot}$ WDs. Model UV and supersoft X-ray light curves are omitted to simplify the figure except three WD masses (blue solid line for 1.0, red solid line for 0.95 , and green solid line for $0.9 M_{\odot}$ ). 
higher mass accretion rates to the WD than that of point $\mathrm{B}$, for the case of V1668 Cyg. This simply suggests that novae are brighter for lower mass accretion rates.

For the other WD masses, the trend between the maximum brightness and the initial envelope mass is the same as that of $0.95 M_{\odot}$. For more (less) massive WDs, the envelope mass at the same point B is smaller (larger) than that of $0.95 M_{\odot}$. We will see again these relations between the maximum brightness and the initial envelope mass in more details in Section 5

We regard that V1668 Cyg is a typical classical nova and point $B$ in Figure 2 corresponds to typical brightnesses for other WD mass models. Therefore, we replot the same nova model light curves in a logarithmic time in Figure 3 . In the overlapping procedure of UV $1455 \AA$ model light curves, we adopt the origin of time which is different from the origin of time in $m_{\mathrm{ff}}$ of Table 2. This procedure is necessary for the horizontal axis of $\log t^{\prime}$ because we must start all the free-free emission light curves from the same stage like point B (or C) in Figure 2. Here we define the origin of time as about 4 days before point B in Figure 2, that is, the outburst day of V1668 Cyg. In this case, the initial brightnesses of novae are almost the same (brightness at point B) and overlapped with each other in the $\log t^{\prime}-m_{V}^{\prime}$ diagram.

\subsection{Absolute magnitude of free-free light curves}

We have given an apparent magnitude to each free-free emission model light curve against V1668 Cyg data. Next step is to determine the absolute magnitudes of these model light curves. This can be done by using the distance modulus of V1668 Cyg. Figure 2 shows observed $y$ and $V$ magnitudes of V1668 Cyg, which can be converted to the absolute magnitude from the distance modulus to V1668 Cyg, i.e.,

$$
(m-M)_{V}=\left[5 \log (d / 10)+A_{V}\right]_{\mathrm{V} 1668 \mathrm{Cyg}}=14.3 .
$$

Here, we adopt the distance of $d=4.1 \mathrm{kpc}$ (Kato \& Hachisu 2007) and the absorption of $A_{V}=3.1 \times E(B-V)=3.1 \times$ $0.4=1.24$ (Stickland et al. 1981) for V1668 Cyg. Since our $0.95 M_{\odot}$ WD model shows a good agreement with the $y$ and $V$ magnitudes of V1668 Cyg, we determine the absolute $V$ magnitude of our $0.95 M_{\odot}$ WD model light curve as follows:

$$
M_{V}=m_{V}-14.3=\left(m_{\mathrm{ff}}+0.9\right)-14.3,
$$

where $m_{V}=m_{V}^{\prime}=m_{\mathrm{ff}}+0.9$ for $0.95 M_{\odot}$ WD model $\left(f_{\mathrm{s}}=1\right)$ from Equation (8). Then, the absolute $V$ magnitude at the last point of free-free template light curve (the end point of Table 2) corresponds to

$$
M_{\mathrm{w}}=m_{\mathrm{w}}-14.3=\left(m_{\mathrm{ff}}+0.9\right)-14.3=1.6,
$$

where the subscript of "w" means "wind" which corresponds to $m_{\mathrm{ff}}=15$ at the end point (Table 2). Using $M_{\mathrm{w}}$, we restore the entire absolute $V$ magnitudes of the $0.95 M_{\odot}$ WD model by

$$
M_{V}=\left(m_{\mathrm{ff}}-15\right)+M_{\mathrm{w}}
$$

and it is shown by a red solid line in Figure 4

For the other WD mass models, from Equation (5) we simply derive the following relations, i.e.,

$$
\begin{aligned}
& M_{V}=m_{V}^{\prime}+2.5 \log f_{\mathrm{s}}-14.3, \\
& M_{\mathrm{w}}=m_{\mathrm{w}}^{\prime}+2.5 \log f_{\mathrm{s}}-14.3,
\end{aligned}
$$

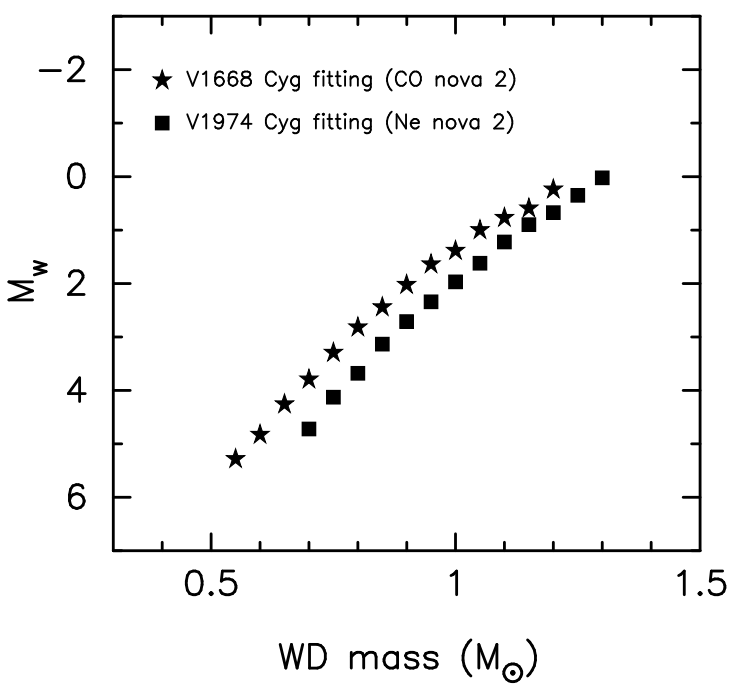

FIG. 5.- The absolute magnitude at the end point of each free-free emission model light curve, $M_{\mathrm{w}}$, is plotted against the WD mass for two different chemical compositions, i.e., "CO nova 2" (star marks) and "Ne nova 2" (squares). See text for details.

based on the V1668 Cyg data. We plot $M_{\mathrm{w}}$ against various WD masses in Figure 5 Using $M_{\mathrm{w}}$, we have

$$
M_{V}=\left(m_{\mathrm{ff}}-15\right)+M_{\mathrm{w}},
$$

where $\log f_{\mathrm{s}}, m_{\mathrm{w}}^{\prime}$, and $M_{\mathrm{w}}$ are tabulated in Table 2 for all other WD mass models from $M_{\mathrm{WD}}=0.55$ to $1.2 M_{\odot}$ by $0.05 M_{\odot}$ step. Here prime symbol means the values for squeezed/stretched light curves. We have restored the entire absolute $V$ magnitudes for $0.55-1.2 M_{\odot}$ WDs in Figure 4

It is interesting to examine whether or not the absolute magnitudes of our free-free emission model light curves are consistent with various empirical relations of classical novae that have ever been proposed. Very popular relations between the maximum magnitude and the rate of decline (MMRD) will be discussed in Section 5. Here we check the empirical formula that the absolute magnitude at 15 days after optical maximum, $M_{V}(15)$, is almost common among various novae (e.g., Buscombe \& de Vaucouleurs 1955; Capaccioli et al. 1989; Cohen 1985; Downes \& Duerbeck 2000; van den Bergh \& Younger 1987). This relation was first proposed by Buscombe \& de Vaucouleurs (1955) with $M_{V}(15)=-5.2 \pm 0.1$, then followed by Cohen (1985) with $M_{V}(15)=-5.60 \pm 0.43$, by van den Bergh \& Younger (1987) with $M_{V}(15)=-5.23 \pm 0.39$, by Capaccioli et al. (1989) with $M_{V}(15)=-5.69 \pm 0.42$, and by Downes \& Duerbeck (2000) with $M_{V}(15)=-6.05 \pm 0.44$. We have obtained $M_{V}(15)=$ $-5.7 \pm 0.3$ for $0.55-1.2 M_{\odot}$ WDs (14 light curves) with equal weight for all WD mass models, being roughly consistent with the above empirical estimates as shown by blue crosses in Figure 4. For the statistical point of view, carbon-oxygen (CO) novae have typical masses of $0.7-1.05 M_{\odot}$. If we take this rage of WD masses (equal weight), the value becomes $M_{V}(15)=-5.95 \pm 0.25$ as indicated in Figure 4, being close to the value proposed by Downes \& Duerbeck (2000).

\subsection{Absolute magnitudes for neon novae}

In this subsection, we study light curves of neon $(\mathrm{Ne})$ novae and show that the essential results on the free-free emission model light curves are the same as those for $\mathrm{CO}$ novae studied in the previous subsections. Table 3 lists free-free model light curves in the form of $m_{\mathrm{ff}}$, definition of which is the same (see 


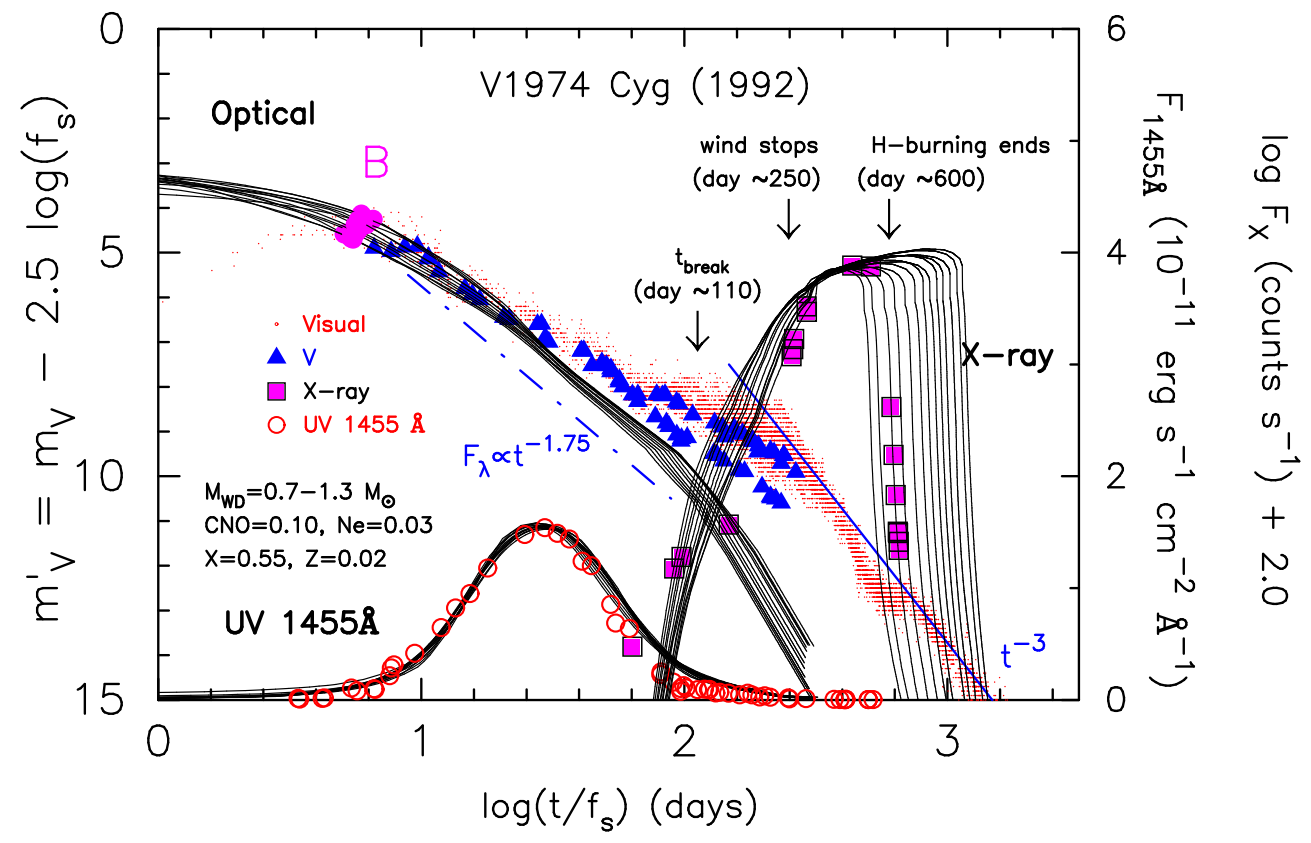

FIG. 6.- Same as Figure 3 but models for "Ne nova 2." Observational data of the neon nova V1974 Cyg is added for comparison. Thirteen different WD mass models $\left(0.7-1.3 M_{\odot}\right.$ by $0.05 M_{\odot}$ step) are plotted in the logarithmic time-magnitude plane, data of which are tabulated in Table 3 We squeeze or stretch the model light curve along time to fit with the observational data of V1974 Cyg, i.e., ROSAT X-ray observation, optical, and IUE UV $1455 \AA$ light curves. UV $1455 \AA$ model fluxes are normalized to have the same peak value as that of $I U E$ data, peak of which is $1.54 \times 10^{-11} \mathrm{erg} \mathrm{s}^{-1} \mathrm{~cm}^{-2} \AA^{-1}$. Fluxes of supersoft X-ray are normalized to fit it with the peak of ROSAT X-ray data. The observational $V$ magnitudes are taken from Chochol et al. (1993), visual data from the American Association of Variable Star Observers (AAVSO), IUE UV $1455 \AA$ data from Cassatella et al. (2002), and ROSAT X-ray data from Krautter et al. (1996). We also added two decline rates of free-free flux, i.e., $F_{\lambda} \propto t^{-1.75}$ (blue dash-dotted line) in the early phase before $t_{\text {break }}$, and $F_{\lambda} \propto t^{-3}$ (blue solid straight line) of Equation [2] in the expanding nebular phase. Here we set point $\mathrm{B}$ on each model light curve near $t_{0}$.

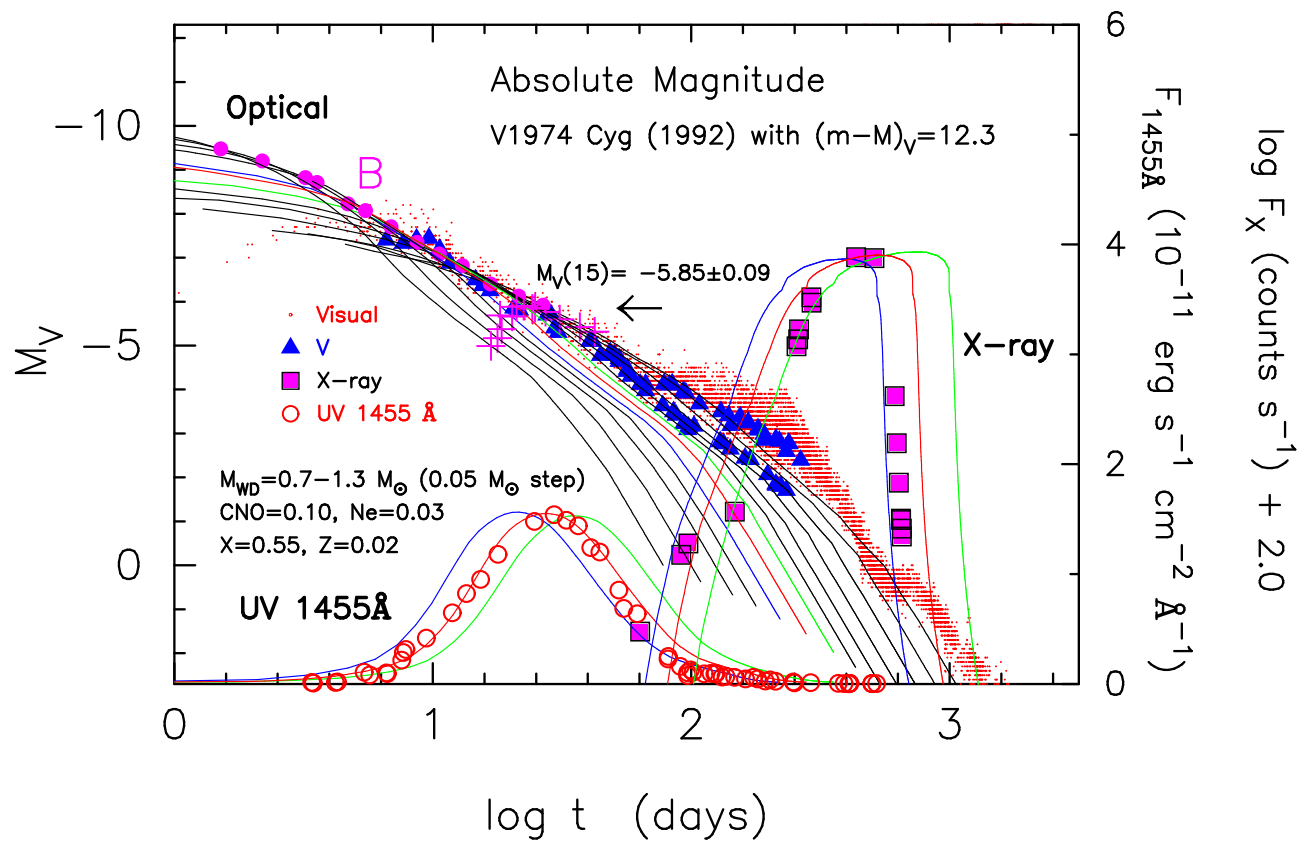

FIG. 7.- Same as Figure 6 but for absolute magnitudes and real timescales. We have calibrated the free-free model light curves by a distance modulus of $(m-M)_{V}=12.3$ for V1974 Cyg. The positions at point B in Figure 6 are indicated by a magenta filled circle. We also show the magnitude, $M_{V}(15)$, at 15 days after the optical maximum by a magenta cross. Their average value of $M_{V}(15)=-5.85 \pm 0.09$ is also indicated in the figure for $0.9-1.15 M_{\odot}$ WDs. Model UV and supersoft X-ray light curves are omitted to simplify the figure except three WD masses (blue solid line for 1.1, red solid line for 1.05, and green solid line for $\left.1.0 M_{\odot}\right)$. 
Equation (3) but for the chemical composition of "Ne nova 2" in Table 1 .

Figure 6 shows free-free (optical), UV $1455 \AA$, and supersoft X-ray model light curves as well as observational data of V1974 Cyg (Nova Cygni 1992). We plot these light curves in $\log t^{\prime}-m_{V}^{\prime}$ plane and show nova evolutions until more later phases. In the overlapping procedure of UV $1455 \AA$ model light curves, we adopt the origin of time which is different from the origin of time in $m_{\mathrm{ff}}$ of Table 3 . This procedure is necessary for the same reason as in Figure 3. As a result, the peak brightness of each light curve is almost the same in the $\log t^{\prime}-m_{V}^{\prime}$ plane as shown in Figure 6 In this figure, we adopt $M_{\mathrm{env}, 0}=1.5 \times 10^{-5} M_{\odot}$ for $1.05 M_{\odot}$ WD model $\left(f_{\mathrm{s}} \approx 1.0\right)$ as a starting point of the model light curves, corresponding to $m_{\mathrm{ff}}=4.1$ of $1.05 M_{\odot}$ WD model in Table 3

We also obtain the absolute magnitudes of free-free model light curves in the same way as the $\mathrm{CO}$ novae mentioned in the previous subsections, but for the neon nova V1974 Cyg as a comparison. Adopting the distance of $d=1.8$ kpc (Chochol et al. 1997) and the absorption of $A_{V}=1.0$ (Chochol et al. 1993) for V1974 Cyg, we obtain the distance modulus of

$$
(m-M)_{V}=\left[5 \log (d / 10)+A_{V}\right]_{\mathrm{V} 1974 \text { Cyg }}=12.3,
$$

for V1974 Cyg. Then, we specify the absolute magnitude at the bottom of our free-free model light curves as

$$
M_{\mathrm{w}}=m_{\mathrm{w}}^{\prime}+2.5 \log f_{\mathrm{s}}-12.3
$$

for V1974 Cyg and tabulate $f_{\mathrm{s}}, m_{\mathrm{w}}^{\prime}$, and $M_{\mathrm{w}}$ in Table 3 We plot $M_{\mathrm{w}}$ for both $\mathrm{CO}$ and Ne novae in Figure 5. This clearly shows that the brightness $M_{\mathrm{w}}$ is smoothly increasing from $\sim 5$ mag to $\sim 0$ mag with $M_{\mathrm{WD}}$ from 0.55 to $1.2 M_{\odot}$ for "CO nova 2" (from 0.7 to $1.3 M_{\odot}$ for "Ne nova 2").

Now, we can determine the absolute magnitude of free-free model light curves, $M_{V}=\left(m_{\mathrm{ff}}-15.0\right)+M_{\mathrm{w}}$ from Table 3, for various WD masses as shown in Figure 7 .

We also check whether or not $M_{V}(15)$ is almost common among various neon novae. We have obtained $M_{V}(15)=$ $-5.6 \pm 0.3$ for $0.70-1.3 M_{\odot}$ WDs (13 light curves with equal weight for all WD mass models), being roughly consistent with the empirical estimates (see Section 3.4). For the statistical point of view, neon novae have typical masses of 0.9 $1.15 M_{\odot}$. If we take this rage of WD masses (equal weight for 6 light curves), the value becomes $M_{V}(15)=-5.85 \pm 0.09$ as indicated in Figure 7.

If we take all the $\mathrm{CO}$ and $\mathrm{Ne}$ nova light curves with equal weight (14+13 WD mass models), the average value becomes $M_{V}(15)=-5.7 \pm 0.3$. Thus, the absolute magnitudes of our free-free emission model light curves are consistent with the $M_{V}(15)$ empirical relations.

\subsection{Consistency check among nova light curves}

We have proposed new methods for obtaining the absolute magnitude of novae in the previous subsections. Using these absolute magnitudes, we can estimate the distance to novae. In this subsection, we will check the accuracy of our new methods by comparing three nova light curves.

First of all, we explain how to select a model that reproduces well observational light curves. Figure 8 shows our method in the case of V1668 Cyg. We shift the model light curve "back and forth" and "up and down" to fit with the observational $y$ magnitudes. Together with the UV $1455 \AA$ light curve, we finally determine by eyes the $0.95 M_{\odot}$ WD as the best-reproducing one among the light curves tabulated in Table 2 ( $\left(0.55-1.2 M_{\odot}\right.$ by $0.05 M_{\odot}$ step). In this figure, we added two other light curves of different WD masses, i.e., 0.85 and $1.05 M_{\odot}$ WDs. Here, we use different phases of the model light curves in Figure 1 to fit with the $y$ observation; the $0.85 M_{\odot}$ WD model is shifted rightward (back) but the $1.05 M_{\odot}$ WD model is shifted leftward (forth). As a result, the three model light curves of $0.85,0.95$, and $1.05 M_{\odot}$ WDs give similar decline rates from maximum until day $\sim 40$. In other words, we can find similar shapes in different phases of different WD mass light curves in Figure 1. In this sense, it is hard to choose the best-reproducing model only from early phase optical and near IR data if neither UV $1455 \AA$ nor Xray data are available.

It should be also noted here that the ambiguity of WD mass estimate of $\mp 0.1 M_{\odot}$ introduces an error of about $\pm 0.4 \mathrm{mag}$ in the distance modulus of $(m-M)_{V}$, when only early optical (or IR) light curves are available. In Figure 8, we obtain ( $m-$ $M)_{V}=m_{\mathrm{w}}-M_{\mathrm{w}}=15.8-1.6=14.3$ for $M_{\mathrm{WD}}=0.95 M_{\odot}$, but $(m-M)_{V}=14.9-1.0=13.9$ for $M_{\mathrm{WD}}=1.05 M_{\odot}$ or $(m-M)_{V}=$ $17.1-2.4=14.7$ for $M_{\mathrm{WD}}=0.85 M_{\odot}$.

Now let us go to the first example of a very fast nova V1500 Cyg (Nova Cygni 1975). Figure 9 demonstrates that optical $V$ (filled triangles), $y$ (filled circles), near infrared $H$ (open squares), and $K$ (open triangles) magnitudes are almost overlapped with each other from $\sim 5$ days until $\sim 100$ days after the outburst. This wavelength-free decline is a characteristic property of free-free emission. This impressive part is reproduced well by Equation (1) with the models of $M_{\mathrm{WD}}=1.05 M_{\odot}$ ("CO nova $\left.2 "\right)$ and $M_{\mathrm{WD}}=1.15 M_{\odot}$ ("Ne nova 2"). V1500 Cyg is a superbright nova, in which the peak luminosity was about 4 mag brighter than the Eddington limit of a $1.0 M_{\odot}$ WD (e.g., Ferland et al. 1986), and the spectra of the superbright part (first 5 days) are approximated by blackbody emission (e.g., Gallagher \& Ney 1976). Therefore, our free-free emission model cannot be applicable to the superbright part.

After day $\sim 70$, the $V$ light curve gradually deviates from the other light curves $(y, H$, and $K$ ) because emission lines dominate the optical fluxes. The $V$ magnitudes are 2 mag or more brighter than the $y, H$, and $K$ magnitudes in the late phase. As extensively discussed in Hachisu \& Kato (2006, 2007) and Hachisu et al. (2008), $V$ magnitudes are often heavily contaminated by strong emission lines such as [O III] $\lambda \lambda$ 4959, 5007 (see Read et al. 2008, for V598 Pup spectra), which eventually dominate over the continuum, causing an increasing deviation from the free-free emission model.

Comparing the apparent magnitude of V1500 Cyg and the absolute magnitude of the model light curve of $1.05 M_{\odot}$ (Table 2), we can estimate the distance modulus. Figure 9 shows that the $1.05 M_{\odot}$ WD model is a best-reproducing model (magenta dash-dotted line) among other WD mass models in Table 2 for chemical composition of "CO nova 2." Using $M_{\mathrm{w}}=1.0$ in Table 2 and $m_{\mathrm{w}}=13.0+0.5=13.5$ from Figure 9 we obtain the distance modulus to V1500 Cyg, i.e., $(m-M)_{V}=$ $m_{\mathrm{w}}-M_{\mathrm{w}}=13.5-1.0=12.5$. If we adopt another chemical composition of "Ne nova 2," we obtain a best-reproducing model of $1.15 M_{\odot}$ WD (black solid line). Then we obtain the distance modulus of $(m-M)_{V}=m_{\mathrm{W}}-M_{\mathrm{W}}=13.6-0.9=12.7$ with $m_{\mathrm{w}}=13.1+0.5=13.6$ (Figure 9) and $M_{\mathrm{w}}=0.9$ (Table 3). The difference between $\mathrm{CO}$ and neon nova models is only $0.2 \mathrm{mag}$. Therefore, this method for obtaining the distance modulus is rather robust against the ambiguity of chemical 


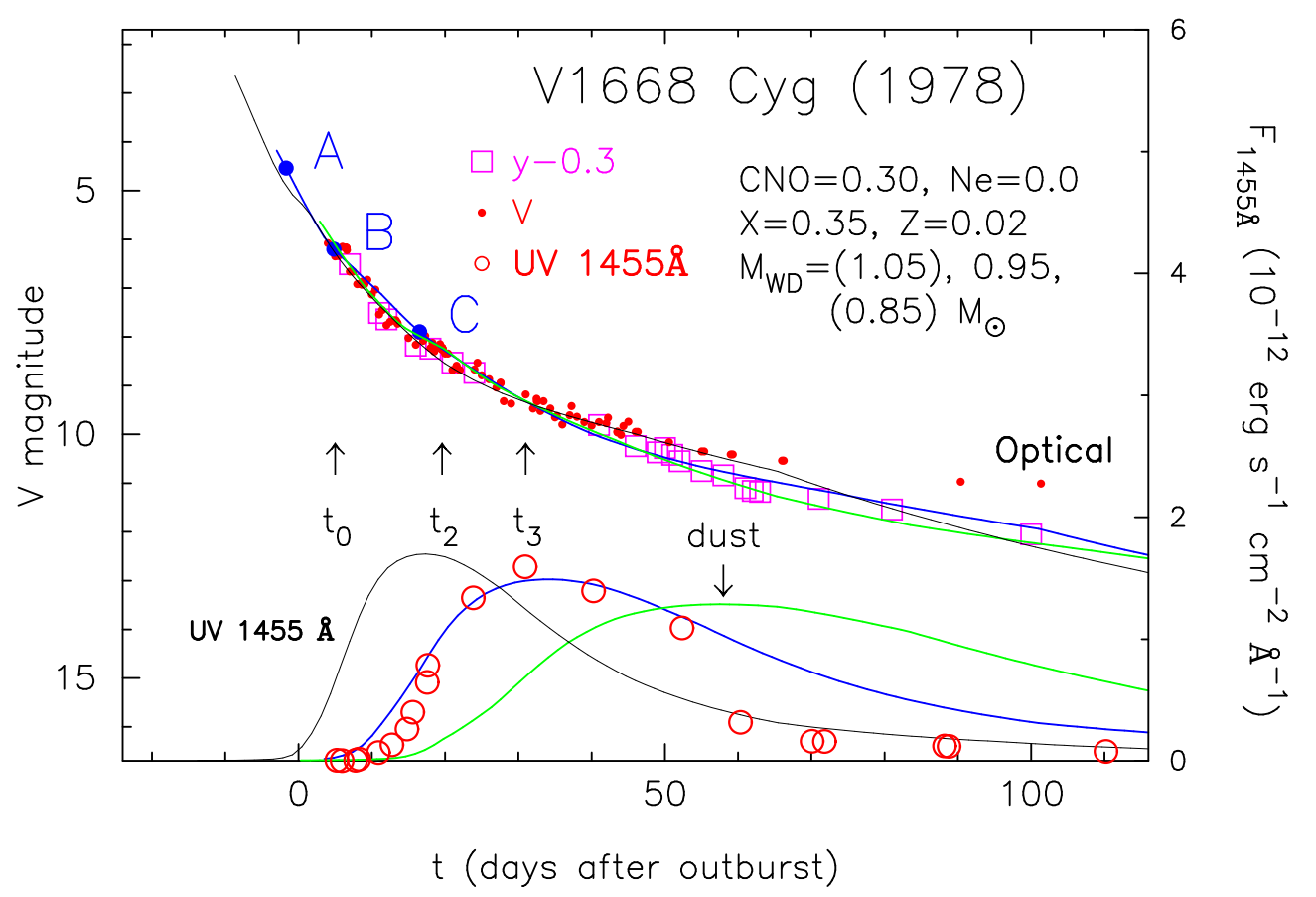

FIG. 8.- Close-up view of three light curves in Figures 1 and 2 Three models are for 1.15 (black thin line), 0.95 (blue thick line), and $0.85 M_{\odot}$ (Green thick line) WDs. None of them are squeezed or stretched. The model of $0.95 M_{\odot}$ WD reasonably reproduces both the optical $y$ (and $V$ ) and UV $1455 \AA$ light curves of V1668 Cyg. In order to fit free-free model light curves of 1.15 and $0.85 M_{\odot}$ WDs with the observation, we shift the $1.05 M_{\odot}$ up and leftward, and the $0.85 M_{\odot}$ down and rightward. As a result, two free-free model light curves reasonably follow the $y$ magnitudes but the two UV light curves do not fit with the observation of V1668 Cyg. Three arrows indicate $t_{0}, t_{2}$, and $t_{3}$ times of V1668 Cyg. The times of $t_{2}$ and $t_{3}$ are determined along our model light curve of the $0.95 M_{\odot}$ WD, which we call "intrinsic" times. Our estimated "intrinsic" values of V1668 Cyg are $t_{2}=14.4$ and $t_{3}=26$ days. We also add observational $V$ magnitudes (red small filled circles), which deviate from $y$ magnitudes in the later phase due mainly to contribution from strong emission lines such as [O III]. Fortunately this deviation has no effects on the estimate of $t_{2}$ and $t_{3}$ times because it starts about 40 days after the outburst in this case. Points A, B, and C correspond to the same initial envelope masses as in Figure 2 along the $0.95 M_{\odot}$ WD model. The magnitude at the end of a wind phase (outside the figure) is $m_{\mathrm{w}}=14.9,15.9$, and 17.1 in this plot, for $M_{\mathrm{WD}}=1.05,0.95$, and $0.85 M_{\odot}$, respectively. So the distance moduli of these three light curves are $(m-M)_{V}=m_{\mathrm{w}}-M_{\mathrm{w}}=14.9-1.0=13.9$, $15.8-1.6=14.3,17.1-2.4=14.7$, respectively.

composition of a nova envelope.

The distance to V1500 Cyg was estimated to be $d=1.5 \pm$ $0.2 \mathrm{kpc}$ from the nebular (ejecta) expansion parallax (e.g., Slavin et al. 1995). The absorption was obtained to be $A_{V}=$ $1.60 \pm 0.16$ by Lance et al. (1988), so that the distance modulus to V1500 Cyg is $(m-M)_{V}=12.5 \pm 0.4$, being consistent with the value of 12.5 ("CO nova 2") and 12.7 ("Ne nova 2") estimated above based on our new method.

Thus, the absolute magnitude estimates of V1500 Cyg based on our free-free emission model light curves are consistent with the distance derived from the nebular expansion parallax method (Slavin et al. 1995) within an accuracy of 0.2 mag in the distance modulus of $(m-M)_{V}$. This in turn supports our assumptions and simplifications on free-free emission of novae.

The second example is GK Per (Nova Persei 1901). We assume that, when two novae have a similar decline rate (or a similar timescale) of free-free light curve, their brightnesses are the same during the period in which the two free-free light curves are overlapped. Figure 10 shows such an example, a comparison between the light curves of GK Per and V1500 Cyg. GK Per has a decline timescale very similar to that of V1500 Cyg. Even in a typical transition phase (from day $\sim 25$ until day $\sim 150$ ), we are able to nicely fit our universal decline law with the observation, especially the bottom line of each oscillation until day $\sim 100$ as shown in the figure. The top line connecting maxima of each oscillation deviates largely from our free-free model light curve. This deviation is caused mainly by the increase in continuum flux itself. The effect of strong emission lines starts from day $\sim 100$, where the bottom line of each oscillation starts to deviate from our model light curve.

Our best-reproducing model is a $1.15 M_{\odot}$ WD among other WD masses in Table 3. Then we obtain the distance modulus of

$$
\left[(m-M)_{V}\right]_{\mathrm{FF}}=m_{\mathrm{w}}-M_{\mathrm{w}}=10.2-0.9=9.3
$$

from $m_{\mathrm{w}}=10.2$ in Figure 10 and $M_{\mathrm{w}}=0.9$ in Table 3 , where FF means the method of our free-free (FF) light curve fitting.

Direct comparison of the brightness between GK Per and V1500 Cyg gives the difference of their distance moduli. From Figure 10, we have

$$
\left[5 \log (d / 10)+A_{V}\right]_{\mathrm{V} 1500 \mathrm{Cyg}}-\left[5 \log (d / 10)+A_{V}\right]_{\mathrm{GK} \text { Per }}=+3.4 \text {, }
$$

where $d$ is the distance in units of pc and $A_{V}$ is the absorption in the $V$ band. With $d=1.5 \mathrm{kpc}$ and $A_{V}=1.6$ for V1500 Cyg and $d=0.455 \mathrm{kpc}($ Slavin et al. 1995$)$ and $A_{V}=3.1 E(B-V)=$ $3.1 \times 0.3=0.9$ (Wu et al. 1989) for GK Per, we obtain

$$
(m-M)_{V}=\left[5 \log (d / 10)+A_{V}\right]_{\mathrm{V} 1500 \mathrm{Cyg}}=12.5,
$$

and

$$
(m-M)_{V}=\left[5 \log (d / 10)+A_{V}\right]_{\text {GK Per }}=9.2 .
$$

Therefore, the difference of $12.5-9.2=3.3$ is consistent with the difference in Equation (19) within an accuracy of $0.1 \mathrm{mag}$ and the distance modulus of Equation (21) is also consistent with the result of the above FF method $(9.3 \mathrm{mag})$ within 0.1 mag. Thus, we confirm that the brightnesses of the two novae are the same within an uncertainty of $\pm 0.2 \mathrm{mag}$. This 


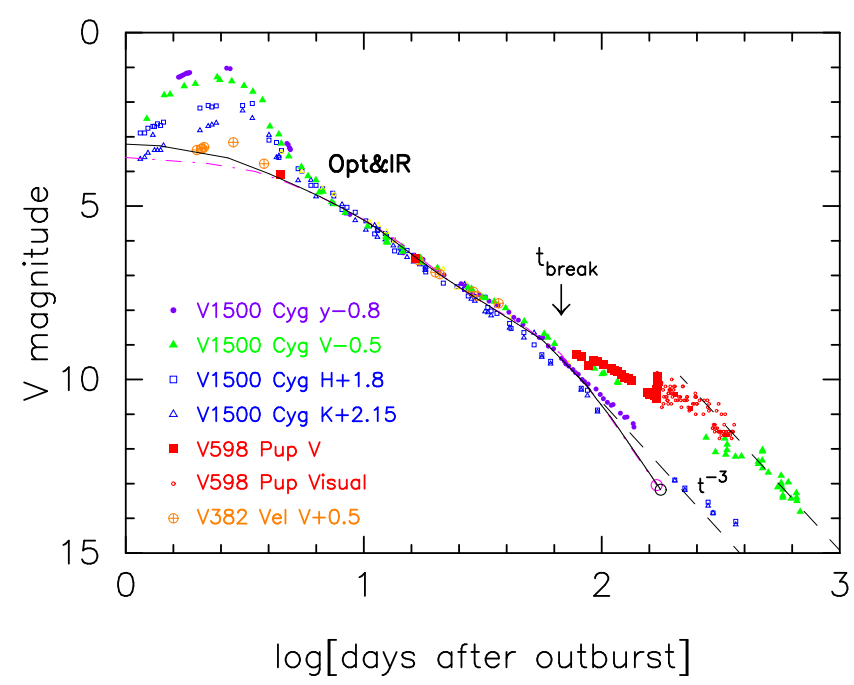

FIG. 9.- Light curves for V1500 Cyg as an example of $t_{\text {break }}$. Optical $V$ (filled triangles), $y$ (filled circles), near infrared (IR) $H$ (open squares), and $K$ (open triangles) magnitudes of the fast nova V1500 Cyg. Light curves of free-free emission are essentially independent of wavelength, so that $V$, $y, H$, and $K$ light curves of V1500 Cyg are almost overlapped with each other from several days to $\sim 70$ days after the outburst. Then, the $V$ light curve deviates from the others $(y, H$, and $K)$ mainly because the wide $V$ band is heavily contaminated by strong emission lines such as [O III]. Two other nova light curves of V598 Pup and V382 Vel show an almost identical decline from several days to several tens of days after the outburst, that is, the free-free part of light curves. Optical $V$ (filled squares) and visual (small open circles) of V598 Pup (see Fig. 16 for more details of V598 Pup) and V (open circles with plus) of V382 Vel (see Fig. 17f for more details of V382 Vel). Black solid line: free-free model light curve of $1.15 M_{\odot}$ WD with a composition of "Ne nova 2." Magenta dash-dotted line: free-free model light curve of $1.05 M_{\odot}$ WD with a composition of "CO nova 2." Large open circle: the epoch when the wind stops. Dashed line: model light curve based on free-free emission following a law of $F_{\lambda} \propto t^{-3}$ (Equation 2] after winds stop). Near IR data of V1500 Cyg are taken from Ennis et al. (1977), Gallagher \& Ney (1976), and Kawara et al. (1976); $y$ and $V$ data of V1500 Cyg are from Lockwood \& Millis (1976) and Tempesti (1979).

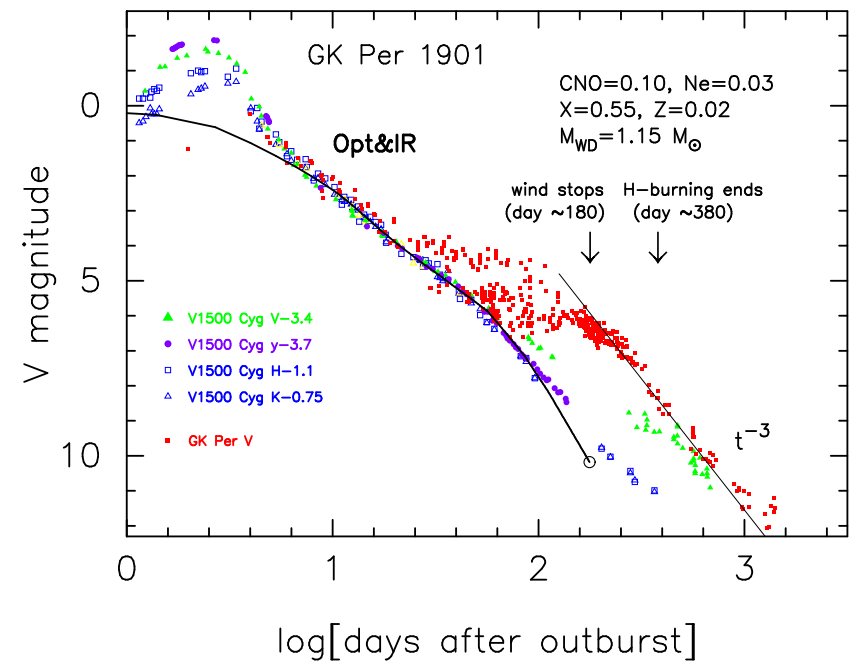

FIG. 10.- Same as Figure 9 but we add the $V$ light curve of GK Per (Nova Persei 1901) for comparison. We fit the smooth light curve of V1500 Cyg with the bottom line of the GK Per light curve during the transition oscillations. Optical $V$ data of GK Per are the same as those in Figure 2 of Hachisu \& Kato (2007).

good agreement supports our assumption that two novae have the same brightness during their overlapping period if the two novae have a similar timescale as shown in Figure 7.

The third example is V1493 Aql (Nova Aquilae 1999
No.1). This nova shows a peculiar secondary peak (Figure 11), which is seen in all the bands, i.e., $V, R$, and $I$ bands. Hachisu \& Kato (2009) explained this kind of secondary peak by an additional energy source from strong magnetic field (and rotation). Therefore, we regard that the nova light curves come back to a free-free emission light curve after the additional energy input stopped. We see that, just after the secondary peak, the light curve sharply drops to merge into the light curve of V1500 Cyg. So we expect that V1493 Aql has a similar decline rate to V1500 Cyg, i.e., our freefree model light curve of $M_{\mathrm{WD}}=1.15 M_{\odot}$ with "Ne nova 2" agrees well with the observation. The effect of strong emission lines starts only from day $\sim 100$, where the $V$ and visual magnitudes begin to depart from the $I$ magnitude.

Using a $1.15 M_{\odot}$ WD model in Table 3 , we obtain the distance modulus of

$$
\left[(m-M)_{V}\right]_{\mathrm{FF}}=m_{\mathrm{w}}-M_{\mathrm{w}}=19.3-0.9=18.4
$$

from $m_{\mathrm{w}}=19.3$ in Figure 11 and $M_{\mathrm{w}}=0.9$ in Table 3

Direct comparison of the brightness between V1493 Aql and V1500 Cyg gives the difference of their distance moduli as shown in Figure 11, i.e.,

$$
\left[5 \log (d / 10)+A_{V}\right]_{\mathrm{V} 1500 \mathrm{Cyg}}-\left[5 \log (d / 10)+A_{V}\right]_{\mathrm{V} 1493 \mathrm{Aql}}=-5.7 .
$$

With $(m-M)_{V}=12.5$ from Equation (20) for V1500 Cyg, we obtain the distance modulus of

$$
\left[(m-M)_{V}\right]_{\mathrm{LC}}=\left[5 \log (d / 10)+A_{V}\right]_{\mathrm{V} 1493 \mathrm{Aql}}=18.2,
$$

where LC means the method of our direct light curve (LC) fitting. The distance modulus of this nova was estimated to be $(m-M)_{V}=17.8$ (Bonifacio et al. 2000), $(m-M)_{V}=18.8$ (Venturini et al. 2004), $(m-M)_{V}=18.4$ (Arkhipova et al. 2002), and $(m-M)_{V}=18.6$ (Burlak 2008), based on the MMRD relations. The simple average values of these four observational estimates becomes $(m-M)_{V}=18.4 \pm 0.4$, being consistent with our estimate of the FF and LC methods. If we adopt $A_{V}=3.1 E(B-V)=3.1 \times 1.5=4.7$ (Arkhipova et al. 2002), the distance is $d=5.5$ and $5.0 \mathrm{kpc}$ for FF and LC method, respectively. These values are consistent with $d=5.4$ kpc obtained by Arkhipova et al. (2002) based on the MMRD relations.

In the present paper, we proposed two new methods for obtaining distance modulus of a nova: one is fitting our free-free (FF) emission model light curve with the observation (we call this FF method). The other is fitting the light curve (LC) of a distance-unknown nova with the other distance-well-known nova (we call this LC method). From the above confirmations, we may conclude that our new methods give a reasonable distance. It should be addressed that our new methods for obtaining distance moduli of novae are applicable even when we missed the optical maxima of nova light curves. In Section 6. we apply these two methods to ten novae.

\section{CHARACTERISTIC TIMESCALES OF NOVA LIGHT CURVES}

There are several timescales that characterize nova light curves; $t_{0}$ is the time in units of day at optical maximum (usually $V$ magnitude); $t_{2}$ and $t_{3}$ are the times in units of day, in which $V$ magnitude decays by 2 and $3 \mathrm{mag}$, respectively, from its maximum (from $t_{0}$ ); $t_{\text {break }}$ is the epoch when our free-free model light curves (or optical $y$, near infrared $I, J, H, K$ light curves) bend sharply as shown in Figures 3 6. and $9 t_{\text {wind }}$ is the epoch when optically thick winds stop; 


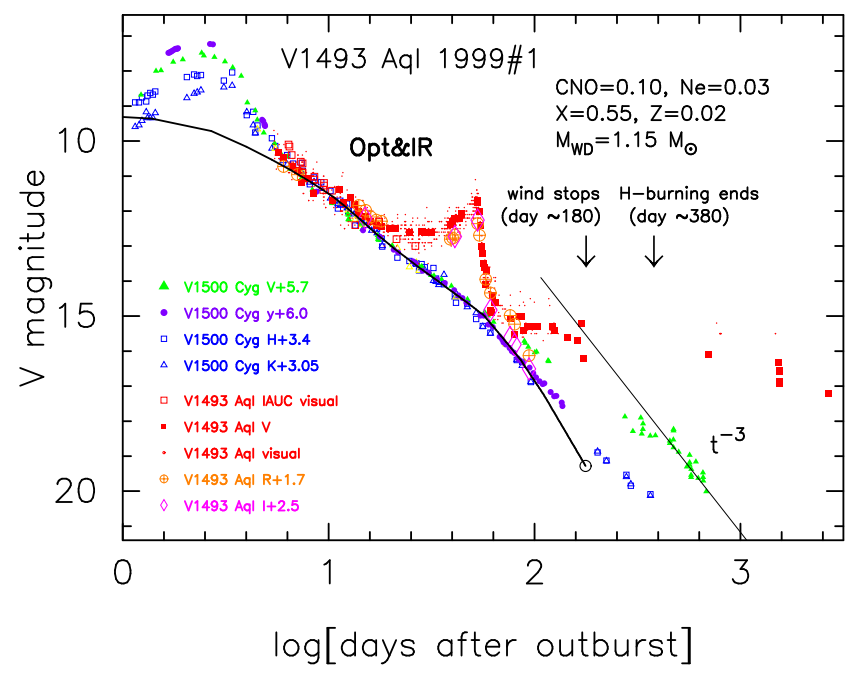

FIG. 11.- Same as Figure 9 but we add light curves of V1493 Aql 1999\#1 for comparison. We fit the smooth light curve of V1500 Cyg with the bottom line of the V1493 Aql light curve. Optical $V$ (filled squares), $R$ (open circles with a plus), I (open diamonds), and visual (very small open circles) are taken from the American Association of Variable Star Observers (AAVSO) and the Variable Star Observers League in Japan (VSOLJ). Some visual magnitude estimates are also taken from IAU Circulars 7225, 7228, 7232, 7258, 7273, and 7313 (open squares). Here we assume the outburst day (day 0 ) of $t_{\mathrm{OB}}=$ JD 2451369.0 (1999 July 9.5 UT) for V1493 Aql.

and $t_{\mathrm{H}-\text { burning }}$ is the epoch when hydrogen shell-burning ends. Hachisu \& Kato (2006, 2007) and Hachisu et al. (2008) calculated free-free model light curves for various WD masses with many sets of chemical composition. They fitted their model light curves with observation and derived $t_{\text {break }}, t_{\text {wind }}$, and $t_{\mathrm{H}-\text { burning }}$ for many novae. Table 4 summarizes such val-

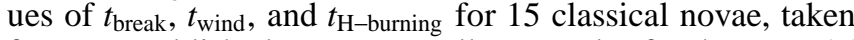
from our published papers as well as $t_{2}$ and $t_{3}$ for the same 15

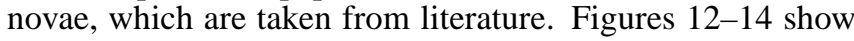
these five timescales for the 15 novae.

When the optically thick wind stops, the photospheric radius shrinks drastically and the photospheric temperature increases up to 20-30 eV (e.g., Hachisu \& Kato 2003) so that the supersoft X-ray flux emerges (see Appendix B for the photospheric radius which is shrinking with the temperature of the WD envelope during a supersoft X-ray phase). We regard that $t_{\text {wind }}$ is almost equal to the emerging time of supersoft $\mathrm{X}$-ray, $t_{\mathrm{X}-\mathrm{on}}$. After the hydrogen shell-burning stops, the WD envelope quickly cools down and the supersoft X-ray phase ends. So we regard that $t_{\mathrm{H}-\text { burning }}$ is almost equal to the decay time of supersoft $\mathrm{X}$-ray, $t_{\mathrm{X} \text {-off. Among these five timescales }}$ of $t_{2}, t_{3}, t_{\text {break }}, t_{\text {wind }}$, and $t_{\mathrm{H} \text {-burning }}$, the first three timescales, $t_{2}, t_{3}$, and $t_{\text {break }}$, are much shorter than the other two, $t_{\text {wind }}$ and $t_{\mathrm{H} \text {-burning. }}$.

\subsection{Relations between various timescales}

In what follows we introduce several relations between these five timescales, $t_{2}, t_{3}, t_{\text {break }}, t_{\text {wind }}$, and $t_{\mathrm{H} \text {-burning. In Fig- }}$ ure 12. we plot $t_{\mathrm{H}-\text { burning }}, t_{\mathrm{wind}}$, and $t_{\text {break }}$ against $t_{\mathrm{break}}$, which are theoretical ones calculated from our free-free model light curves (Hachisu \& Kato 2006, 2007). These values depend on $M_{\mathrm{WD}}$ and the chemical composition, but are actually independent of $M_{\mathrm{env}, 0}$. This is because the difference in $M_{\mathrm{env}, 0}$ makes a large difference in the optical maximum but affects only the very early phase of nova outbursts as shown in Figures 2 and 8 and the difference in the epoch of optical maximum is so short (a few to several days) that it can be neglected compared with the timescales of $t_{\mathrm{H} \text {-burning }}, t_{\mathrm{wind}}$, and $t_{\text {break }}$ (several tens of days or a few hundred days). So we can derive relations among $t_{\mathrm{H}-\text { burning }}, t_{\text {wind }}$, and $t_{\text {break }}$ from our models (independently of observations) as shown in Figures 12 14. Once we determine $t_{\text {break }}$ of individual novae from observations, we can theoretically predict the duration of a luminous supersoft X-ray phase from Figure 12

From Figure 12, we obtain approximate relations between $t_{\text {break }}$, turn-on $\left(t_{\mathrm{X}-\mathrm{on}} \approx t_{\text {wind }}\right)$, and turnoff $\left(t_{\mathrm{X} \text {-off }} \approx t_{\mathrm{H} \text {-burning }}\right)$ times as

$$
\begin{gathered}
t_{\mathrm{X}-\mathrm{on}} \approx t_{\text {wind }}=(2.6 \pm 0.4) t_{\text {break }}, \\
t_{\mathrm{X}-\text { off }} \approx t_{\mathrm{H}-\text { burning }}=(0.71 \pm 0.1)\left(t_{\text {break }}\right)^{1.5}
\end{gathered}
$$

and

$$
t_{\mathrm{X}-\mathrm{off}} \approx t_{\mathrm{H}-\text { burning }}=(0.17 \pm 0.023)\left(t_{\mathrm{wind}}\right)^{1.5},
$$

for $25 \lesssim t_{\text {break }} \lesssim 250$ days.

It should be noted that the proportionality of $t_{\text {wind }}$ to $t_{\text {break }}$, i.e., $t_{\text {wind }} / t_{\text {break }} \approx 2.6$, is a direct result of our scaling law (the universal decline law), because both $t_{\text {break }}$ and $t_{\text {wind }}$ are during the optically thick wind phase which is the subject of universal decline law. Then the $t_{\text {wind }}$ is directly proportional to $t_{\text {break }}$. On the other hand, the turnoff time of hydrogen shell burning, $t_{\mathrm{H}-\text { burning }}$, is not proportional to $t_{\text {break }}$ or $t_{\text {wind }}$, because the duration of static hydrogen shell burning phase depends on hydrogen content $X$ (amount of fuel) of the envelope and WD mass (temperature) in a different way from the optically thick wind phase, in which the decay rate is determined mainly by wind mass-loss not by hydrogen burning rate (see Figure 6). Therefore, Equations (26) and 27) are empirical laws obtained numerically, the power of which is not 1.0 but approximately 1.5 .

It is, however, not easy to obtain $t_{\text {break }}$ because nova light curves such as $V$ magnitudes are often heavily contaminated by strong emission lines in the later phase and this contamination easily clouds the break point of light curves as can be seen in Figure 9. In the next subsection, we derive relations between $t_{3}$ time and two of $t_{\text {wind }}$ and $t_{\mathrm{H}-\text { burning }}$ times, because $t_{3}$ time is determined relatively easily from early optical observation.

\subsection{Prediction formulae by intrinsic $t_{3}$ time}

It is very useful if we can predict $t_{\mathrm{X} \text {-on }}\left(\approx t_{\text {wind }}\right)$ and $t_{\mathrm{X} \text {-off }}$ ( $\approx t_{\mathrm{H} \text {-burning }}$ ) from $t_{3}$ time, because $t_{3}$ is popular and widely used to define the nova speed class. In this subsection, we derive relations between $t_{3}$ and $t_{\mathrm{X}-\mathrm{on}}$, and between $t_{3}$ and $t_{\mathrm{X}-\mathrm{off}}$.

As already explained in the previous subsection, $t_{\text {break }}, t_{\text {wind }}$, and $t_{\mathrm{H}-\text { burning }}$ are practically independent of the initial envelope mass, $M_{\text {env, } 0}$, but $t_{3}$ time depends on the peak brightness of an individual nova. As shown in Figures 2 and 8 , the initial envelope mass $M_{\mathrm{env}, 0}$ is larger, the optical maximum is brighter. Therefore, $t_{2}$ and $t_{3}$ depend on $M_{\text {env, }, 0}$. This is the reason why we cannot theoretically derive $t_{2}$ and $t_{3}$. We need information of maximum brightness to specify $t_{3}$ time for individual novae.

Now we go back to the well-observed V1668 Cyg which we regard as a typical classical nova. Thus, we adopt point B in Figures 2 and 8 as a typical (an average) case of maximum brightness. In this case, $t_{2}=14.4$ and $t_{3}=26$ days are derived from Figures 2 and 8 . Then, we have $t_{3} / t_{\text {break }}=26 / 100$ (see Table 6 for V1668 Cyg timescales). Using the universal decline law, we obtain a relation between $t_{3}$ and $t_{\text {break }}$ as

$$
\frac{t_{3}}{t_{\text {break }}}=\frac{t_{3} / f_{\mathrm{s}}}{t_{\text {break }} / f_{\mathrm{s}}}=\frac{26 \pm 4}{100}=0.26 \pm 0.04,
$$


TABLE 4

Light CuRve PARAMETERS OF NOVAe IN Literature

\begin{tabular}{llrrrrrr}
\hline \hline \multicolumn{1}{c}{ object } & $\begin{array}{c}t_{2} \\
\text { (days) }\end{array}$ & $\begin{array}{c}t_{3} \\
\text { (days) }\end{array}$ & ref. $^{\text {a }}$ & $\begin{array}{c}t_{\text {break }} \\
\text { (days) }\end{array}$ & $\begin{array}{c}t_{\text {wind }} \\
\text { (days) }\end{array}$ & $\begin{array}{c}t_{\text {H-burning }} \\
\text { (days) }\end{array}$ & ref. $^{\text {b }}$ \\
\hline GK Per 1901 & 7 & 13 & 1 & 73 & 182 & 382 & 5 \\
V1500 Cyg 1975 & 2.9 & 3.6 & 2 & 70 & 180 & 380 & 8 \\
V1668 Cyg 1978 & 12.2 & 24.3 & 3 & 110 & 280 & 720 & 8 \\
V1974 Cyg 1992 & 16 & 42 & 1 & 96 & 250 & 600 & 8 \\
V382 Vel 1999 & 6 & 12 & 4 & 49 & 120 & 220 & 9 \\
V2361 Cyg 2005 & 6 & 8 & 5 & 69 & 169 & 340 & 5 \\
V382 Nor 2005 & 12 & 18 & 5 & 73 & 182 & 382 & 5 \\
V5115 Sgr 2005 & 7 & 14 & 5 & 60 & 145 & 280 & 5 \\
V378 Ser 2005 & 44 & 90 & 5 & 257 & 858 & 2560 & 5 \\
V5116 Sgr 2005\#2 & 20 & 33 & 5 & 114 & 319 & 757 & 5 \\
V1188 Sco 2005 & 7 & 21 & 5 & 44 & 110 & 190 & 5 \\
V1047 Cen 2005 & 6 & 26 & 5 & 257 & 858 & 2560 & 5 \\
V476 Sct 2005 & 15 & 28 & 6 & 100 & 260 & 590 & 5 \\
V1663 Aq1 2005 & 13 & 26 & 5 & 100 & 260 & 590 & 5 \\
V477 Sct 2005\#2 & 3 & 6 & 7 & 32 & 80 & 121 & 5 \\
\hline
\end{tabular}

${ }^{a} t_{2}$ and $t_{3}$ times at observational face value taken from literature of 1 Downes \& Duerbeck (2000), 2.Warner (1995), 3-Mallama \& Skillman (1979), 4 Vanlandingham et al. (2001), 5Hachisu \& Kato (2007), 6-Munari et al. (2006b), 7. Munari et al. (2006a)

${ }^{\mathrm{b}} t_{\text {break }}, t_{\text {wind }}$, and $t_{\mathrm{H} \text {-burning }}$ taken from 5 . Hachisu \& Kato (2007), 8. Hachisu \& Kato (2006), 9present work

TABLE 5

Prediction Formulae vs. Nova Decay Timescales in Literature

\begin{tabular}{|c|c|c|c|c|c|c|c|}
\hline object & $\begin{array}{l}\ldots \\
\ldots\end{array}$ & $\begin{array}{c}t_{2} \\
\text { (day) }\end{array}$ & $\begin{array}{c}t_{3} \\
\text { (day) }\end{array}$ & $\begin{array}{c}t_{2}-t_{3} \\
\text { (eq. 29]) }\end{array}$ & $\begin{array}{l}t_{3}-t_{\text {Xon }} \\
\text { (eq. [30]) }\end{array}$ & $\begin{array}{l}t_{3}-t_{\text {Xoff }} \\
\text { (eq. 31]) }\end{array}$ & reference $^{\mathrm{a}}$ \\
\hline V598 Pup 2007\#2 & $\ldots$ & - & - & - & - & - & - \\
\hline V382 Vel 1999 & $\ldots$ & 7.5 & 15 & $<$ yes $>^{b}$ & _- & no & Burlak (2008) \\
\hline V382 Vel 1999 & $\ldots$ & 4.5 & 9 & $<$ yes $>$ & - & $<$ yes $>$ & Della Valle et al. (2002) \\
\hline V382 Vel 1999 & $\ldots$ & 6 & 12 & yes & - & yes & Vanlandingham et al. (2001) \\
\hline V382 Vel 1999 & $\ldots$ & - & 12.5 & - & _- & yes & Liller \& Jones (2000) \\
\hline V382 Vel 1999 & $\ldots$ & 6 & 10 & yes & _- & yes & Della Valle et al. (1999) \\
\hline V4743 Sgr 2002\#3 & $\ldots$ & 12 & 22 & yes & - & $<$ yes $>$ & Burlak (2008) \\
\hline V4743 Sgr $2002 \# 3$ & $\ldots$ & - & 15 & - & - & $<$ yes $>$ & Nielbock \& Schmidtobreick (2003) \\
\hline V4743 Sgr $2002 \# 3$ & $\ldots$ & 9 & 16 & yes & - & yes & Morgan et al. (2003) \\
\hline V1281 Sco $2007 \# 2$ & $\ldots$ & - & - & - & - & - & - \\
\hline V597 Pup 2007\#1 & $\ldots$ & 2.5 & - & - & - & - & Naik et al. (2009) \\
\hline V1494 Aql 1999\#2 & $\ldots$ & 8 & 16 & $<$ yes $>$ & no & no & Burlak (2008) \\
\hline V1494 Aql 1999\#2 & $\ldots$ & 7 & 23 & no & yes & $<$ yes $>$ & Venturini et al. (2004) \\
\hline V1494 Aq1 1999\#2 & $\ldots$ & 6.1 & 15.8 & no & no & no & Barsukova \& Goranskii (2003) \\
\hline V1494 Aq1 1999\#2 & $\ldots$ & 6 & 15 & no & no & no & Arkhipova et al. (2002) \\
\hline V1494 Aq1 1999\#2 & $\ldots$ & 6.6 & 16 & no & no & no & Kiss \& Thomson (2000) \\
\hline V2467 Cyg 2007 & $\ldots$ & 7.3 & 15.1 & $<$ yes $>$ & - & no & Lynch et al. (2009) \\
\hline V2467 Cyg 2007 & $\ldots$ & 7.6 & 14.6 & yes & _- & no & Poggiani (2009) \\
\hline V2467 Cyg 2007 & $\ldots$ & 8 & - & - & - & - & Senziani et al. (2008) \\
\hline V5116 Sgr $2005 \# 2$ & $\ldots$ & 6.5 & 20.2 & no & _- & no & Dobrotka et al. (2008) \\
\hline V5116 Sgr $2005 \# 2$ & $\ldots$ & 18 & 45 & no & - & no & Burlak (2008) \\
\hline V5116 Sgr 2005\#2 & $\ldots$ & 20 & 33 & yes & - & yes & Hachisu \& Kato (2007) \\
\hline V574 Pup 2004 & $\ldots$ & 37 & 85 & no & no & no & Burlak (2008) \\
\hline V574 Pup 2004 & $\ldots$ & 13 & 58 & no & no & no & Siviero et al. (2005) \\
\hline V458 Vul 2007 & $\ldots$ & 8 & 31 & no & no & - & Wesson et al. (2008) \\
\hline V458 Vul 2007 & $\ldots$ & 7 & 15 & $<$ yes $>$ & no & _- & Poggiani (2008) \\
\hline V458 Vul 2007 & $\cdots$ & 7 & 18 & no & no & - & Tarasova $(2008)$ \\
\hline V1974 Cyg 1992 & $\ldots$ & 22 & 48 & no & no & no & Burlak (2008) \\
\hline V1974 Cyg 1992 & $\ldots$ & 16 & 42 & no & no & no & Warner (1995) \\
\hline V1668 Cyg 1978 & $\ldots$ & 12.2 & 24.3 & yes & - & - & Mallama \& Skillman (1979) \\
\hline V1500 Cyg 1975 & $\ldots$ & 2.9 & 3.6 & - & - & - & Warner (1995) \\
\hline
\end{tabular}

a reference for quoted values of $t_{2}$ and $t_{3}$ at observational face value

b "yes" satisfies eqs. 29, 30, and 31 but "no" does not and parenthesis means slightly outside the border 


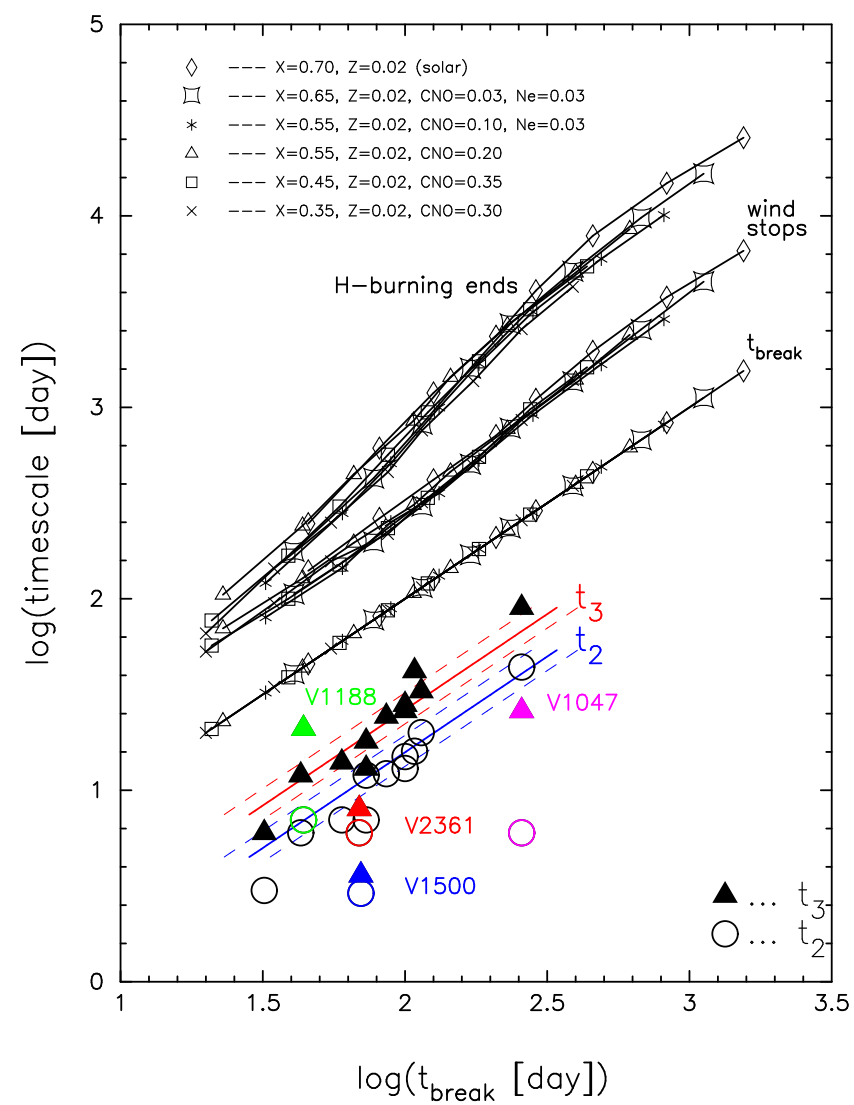

FIG. 12.- Five characteristic nova timescales against $t_{\text {break }}$. From top to bottom, hydrogen shell-burning $\left(t_{\mathrm{H}-\text { burning }}\right)$, optically thick wind $\left(t_{\text {wind }}\right)$, break point of nova light curve $\left(t_{\text {break }}\right), t_{3}$, and $t_{2}$. Supersoft X-ray on/off correspond to $t_{\text {wind }}$ and $t_{\mathrm{H}-\text { burning }}$, respectively. Each symbol in the upper left corner represents our various WD models with different chemical compositions taken from Hachisu \& Kato (2006). We also plot observational face values of $t_{3}$ (filled triangles) and $t_{2}$ (open circles) for 15 novae in Table 4 Thick red solid line: central values of Equation (28). Upper/lower red dashed lines: upper/lower limit values of Equation $(28)$. Thick blue solid line: central values of $t_{2}-t_{\text {break }}$ relation. Upper/lower blue dashed lines: upper/lower values of $t_{2}-t_{\text {break }}$ relation. Colored marks indicate $t_{2}$ and $t_{3}$ times that deviate largely from the statistical relations. Green: visual magnitudes of V1188 Sco are so contaminated by emission lines that $t_{3}$ is largely deviated from the central line. Red: visual and $V$ light curves of V2361 Cyg are affected by dust formation. Blue: V1500 Cyg is a superbright nova and its face values of $t_{2}$ and $t_{3}$ times do not follow the universal decline law. Magenta: visual and $V$ light curves of V1047 Cen is also a superbright nova.

for $8 \lesssim t_{3} \lesssim 80$ days, where the upper and lower bounds are corresponding to $\pm 0.5 \mathrm{mag}$ brighter/darker (more massive/less massive initial envelope mass) points than that of point $\mathrm{B}$. This relation is indicated by a red thick solid line in Figure 12 together with the upper and lower red dashed lines that denote upper/lower values $( \pm 0.04)$ of Equation (28).

There is a relation between $t_{2}$ and $t_{3}$ derived from the scaling law of our universal decline law, which is also well used in literature. Hachisu \& Kato (2007) have already derived

$$
t_{2}=(0.6 \pm 0.08) t_{3},
$$

for the optical light curves that follow the free-free model light curve with $8 \lesssim t_{3} \lesssim 80$ days. This relation has been empirically known (Capaccioli et al. 1990) and theoretically confirmed by Hachisu \& Kato (2006) based on the universal decline law with a slope of $F_{\lambda} \propto t^{-1.75}$ as shown in Figures 3 and 6 Inserting the central value of Equation (29) into Equation (28), we obtain relations between $t_{\text {break }}$ and $t_{2}$ relations by blue solid and dashed lines as shown in Figure 12.

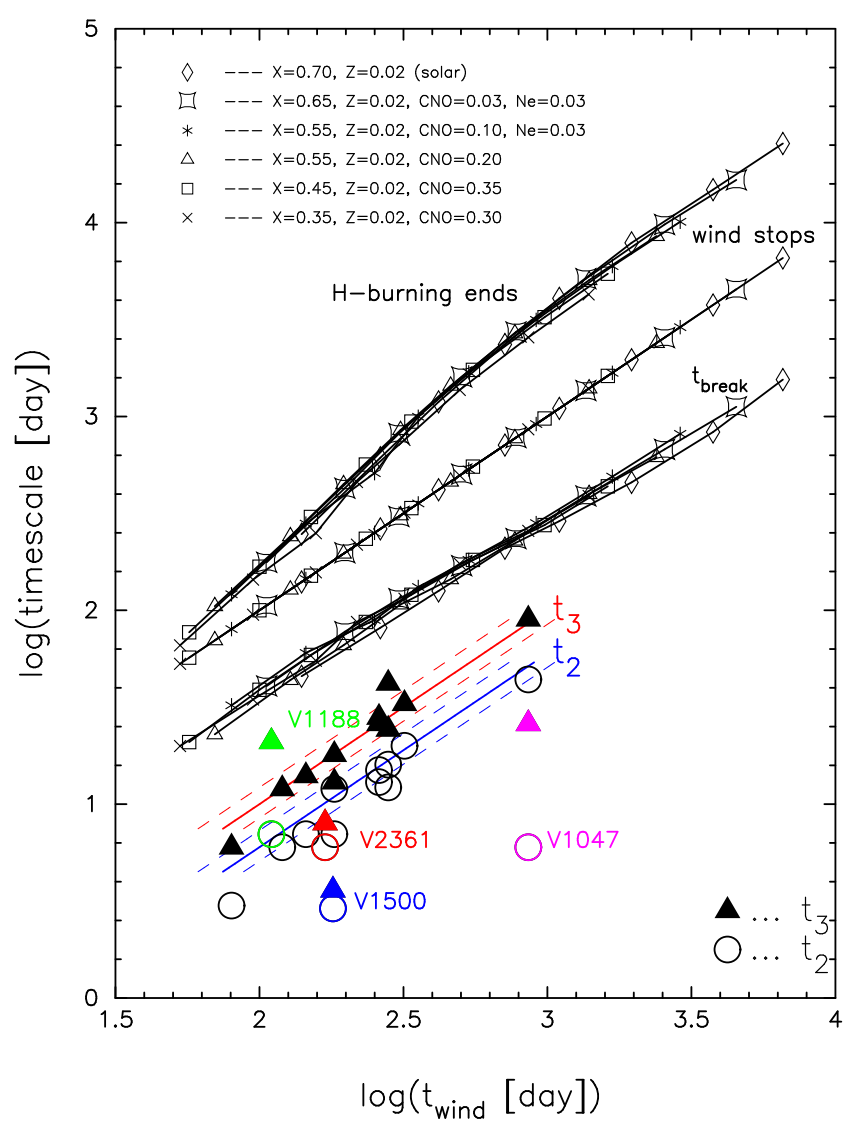

FIG. 13.- Same as Figure 12 but against $t_{\text {wind }}$. Thick red solid line: central values of Equation (30). Upper/lower red dashed lines indicate upper/lower limit values of Equation 30. Thick blue solid line: central values of $t_{2}-t_{\text {wind }}$ relation. Upper/lower blue dashed lines: upper/lower values of $t_{2}-t_{\text {wind }}$ relation. See text for more details.

Figure 12 also shows $t_{2}$ and $t_{3}$ times for individual novae in Table 4 Four novae indicated by colors are far from the relation of Equation (28), but there is a special reason in each nova as described in the figure caption. The other novae reasonably follow the above relation with the reasonable scatter corresponding to $\pm 0.5 \mathrm{mag}$ brighter/darker of the peak brightness. This supports that V1668 Cyg has an average $M_{\text {env }, 0}$.

In the same way, we derive a relation between $t_{3}$ and $t_{\text {wind }}$ from Equations (25) and (28), i.e.,

$$
t_{\mathrm{X}-\text { on }} \approx t_{\text {wind }}=(10.0 \pm 1.8) t_{3}
$$

for $8 \lesssim t_{3} \lesssim 80$ days. Figure 13 shows the five timescales against $t_{\text {wind }}$ and we also see a reasonable fit of our relation (Equation (30)) with the observation.

From Equations (26) and (28), we derive a relation between $t_{3}$ and $t_{\mathrm{H}-\text { burning }}$, i.e.,

$$
t_{\mathrm{X}-\text { off }} \approx t_{\mathrm{H}-\text { burning }}=(5.3 \pm 1.4)\left(t_{3}\right)^{1.5},
$$

for $8 \lesssim t_{3} \lesssim 80$ days. Figure 14 shows the five timescales against $t_{\mathrm{H} \text {-burning }}$ and we can also see a reasonable fit of Equation (31) with the observation.

However, it is not always easy to determine $t_{3}$ (or $t_{2}$ ) time from observation. In fact, Table 5 shows nova timescales $t_{2}$ and $t_{3}$ of 11 novae taken from literature, which are sometimes very different from author to author, and sometimes very different from our estimates in Table 6. Therefore, we propose a more robust way. Instead of observational face values of $t_{2}$ and $t_{3}$, we will use "intrinsic" $t_{2}$ and $t_{3}$, which are obtained 


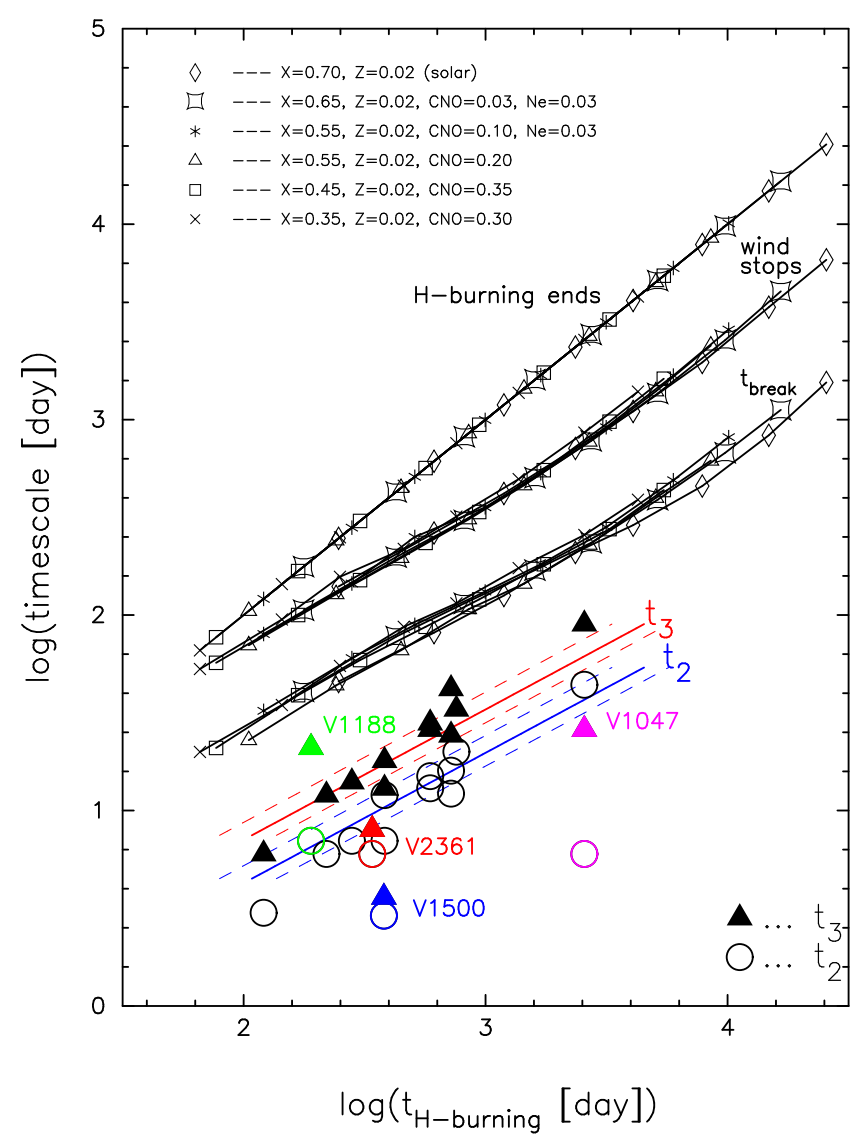

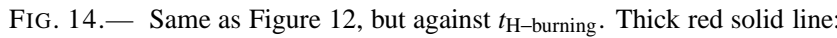
central values of Equation (31). Upper/lower red dashed lines indicate upper/lower limit values of Equation (31). Thick blue solid line: central values of $t_{2}-t_{\mathrm{H} \text {-burning }}$ relation. Upper/lower blue dashed lines: upper/lower values of $t_{2}-t_{\mathrm{H}-\text { burning }}$ relation. See text for more details.

from our model light curves fitted with observation. For example, we use $t_{2}=14.4$ and $t_{3}=26$ days for V1668 Cyg obtained from Figures 2 and 8 instead of observational face values. This "intrinsic" $t_{2}$ and $t_{3}$ times are especially useful when the observational data deviate from our model light curve near the peak. In what follows, we will see how to determine "intrinsic" $t_{3}$.

Figure 8 explained how to select our best model light curve from early phase observations. It also shows that three different WD mass $\left(0.85,0.95\right.$, and $\left.1.05 M_{\odot}\right)$ models can reproduce the early phase light curve and we can hardly select one only from the early phase optical and IR light curves. However, this uncertainty of mass determination is not a problem in the estimation of "intrinsic" $t_{3}$ time, because these three model light curves, of course, give similar values of $t_{3}$ time $\left(t_{3} \sim 26 \pm 2\right.$ days) along our model light curves.

Using relations of Equations (25)-(31), we can predict the period of a supersoft X-ray phase of individual novae from $t_{3}$ (or $t_{2}, t_{\text {break }}$ ) time. In Section 6, we will show that our formulae are much better fitted with observation if we use intrinsic $t_{3}$ time than if we use face values of $t_{3}$. Therefore, in what follows in this paper, we use intrinsic $t_{3}$ time in order to predict $t_{\mathrm{X}-\text { on }} \approx t_{\text {wind }}$ and $t_{\mathrm{X} \text {-off }} \approx t_{\mathrm{H}-\text { burning from Equations }}$ and (31).

We assumed here that a nova has a typical $M_{\text {env, } 0}$, i.e., has a peak brightness similar to that of V1668 Cyg (i.e., point B in the rescaled $t / f_{\mathrm{s}}-m_{V}^{\prime}$ plane, Figure 2). If the rescaled peak magnitude is much brighter/darker than that of V1668 Cyg (for example, points A/C in Figure 2), X-ray on and off times, $t_{\mathrm{X} \text {-on }} \approx t_{\text {wind }}$ and $t_{\mathrm{X}-\text { off }} \approx t_{\mathrm{H} \text {-burning }}$, can deviate from the prediction of Equations (30) and (31). In this sense, our prediction formulae (30) and (31) are statistical ones.

\section{MMRD RELATION DERIVED FROM A UNIVERSAL DECLINE LAW}

The Maximum Magnitude-Rate of Decline (MMRD) relation is often used to estimate the distance to novae (e.g., Cohen 1988; Della Valle \& Livio 1995; de Vaucouleurs 1978; Downes \& Duerbeck 2000; Schmidt 1957). In this section, we derive theoretical MMRD relations based on our universal decline law.

\subsection{Average MMRD relation}

Figures 4 and 7 clearly shows a trend that a more massive WD has a brighter maximum magnitude ( $\operatorname{smaller} M_{V, \max }$ ) and a faster decline rate (smaller $t_{3}$ time). The relation between $t_{3}$ and $M_{V \text {,max }}$ for novae is called "the Maximum MagnitudeRate of Decline" (MMRD) relation. In order to derive theoretical MMRD relations, we go back to Figure 2 and utilize the universal decline law starting from point B (a typical case of nova envelope mass).

We have already shown that all the free-free emission model light curves for different WD masses overlap with each other (they converge to $0.95 M_{\odot}$ WD in Figure 2 against V1668 Cyg data) when they are squeezed in the direction of time by a factor of $f_{\mathrm{s}}$, data of which are tabulated in Table 2 Now we assume that all the free-free emission model light curves reaches the same maximum point, like point B in Figure 2 In such a case, the apparent maximum brightness $m_{V \text {, max }}$ of each light curve with different WD masses is expressed as $m_{V, \text { max }}=m_{V, \text { max }}^{\prime}+2.5 \log f_{\mathrm{s}}$. The $t_{3}$ time of each model light curve with different WD masses is squeezed to be $t_{3}=f_{\mathrm{s}} t_{3}^{\prime}$. Eliminating $f_{\mathrm{s}}$ from these two relations, we have

$$
m_{V, \max }=2.5 \log t_{3}+m_{V, \text { max }}^{\prime}-2.5 \log t_{3}^{\prime} .
$$

Since point B corresponds to the optical peak of V1668 Cyg $\left(f_{\mathrm{s}}=1\right.$ in Figure 2), we have already known $t_{3}^{\prime}=26$ days and $m_{V, \text { max }}^{\prime}=6.2$. Substituting these values of $\left(t_{3}^{\prime}=26, m_{V, \text { max }}^{\prime}=\right.$ 6.2) into Equation (32), we have

$$
m_{V, \max }=2.5 \log t_{3}+6.2-2.5 \log 26=2.5 \log t_{3}+2.7 .
$$

This is our theoretical apparent MMRD ration for a starting point B in Figure 2. Next, using the distance modulus of ( $m-$ $M)_{V}$, we obtain our theoretical MMRD relation as

$$
\begin{aligned}
M_{V, \text { max }} & =m_{V, \max }-(m-M)_{V} \\
& =2.5 \log t_{3}+m_{V, \text { max }}^{\prime}-2.5 \log t_{3}^{\prime}-(m-M)_{V} .
\end{aligned}
$$

Substituting $(m-M)_{V}=14.3$, the distance modulus of V1668 Cyg, into Equation (34), we have

$$
M_{V, \text { max }}=2.5 \log t_{3}+6.2-2.5 \log 26-14.3=2.5 \log t_{3}-11.6 \text {. }
$$

Figure 15 shows this theoretical MMRD relation for novae that has a typical (average) $M_{\mathrm{env}, 0}$ like V1668 Cyg. In the right axis we convert the apparent magnitude to the absolute magnitude by using the distance modulus of V1668 Cyg. Point B in Figure 15 corresponds to V1668 Cyg $\left(f_{\mathrm{s}}=1: 0.95 M_{\odot}\right.$, in which nova light curves start from point B in Figure 2). The other WD mass cases starting from the same point B in Figure 2 have different $f_{\mathrm{s}} \neq 1$. Changing $f_{\mathrm{s}}$ (or $M_{\mathrm{WD}}$ ), we have 


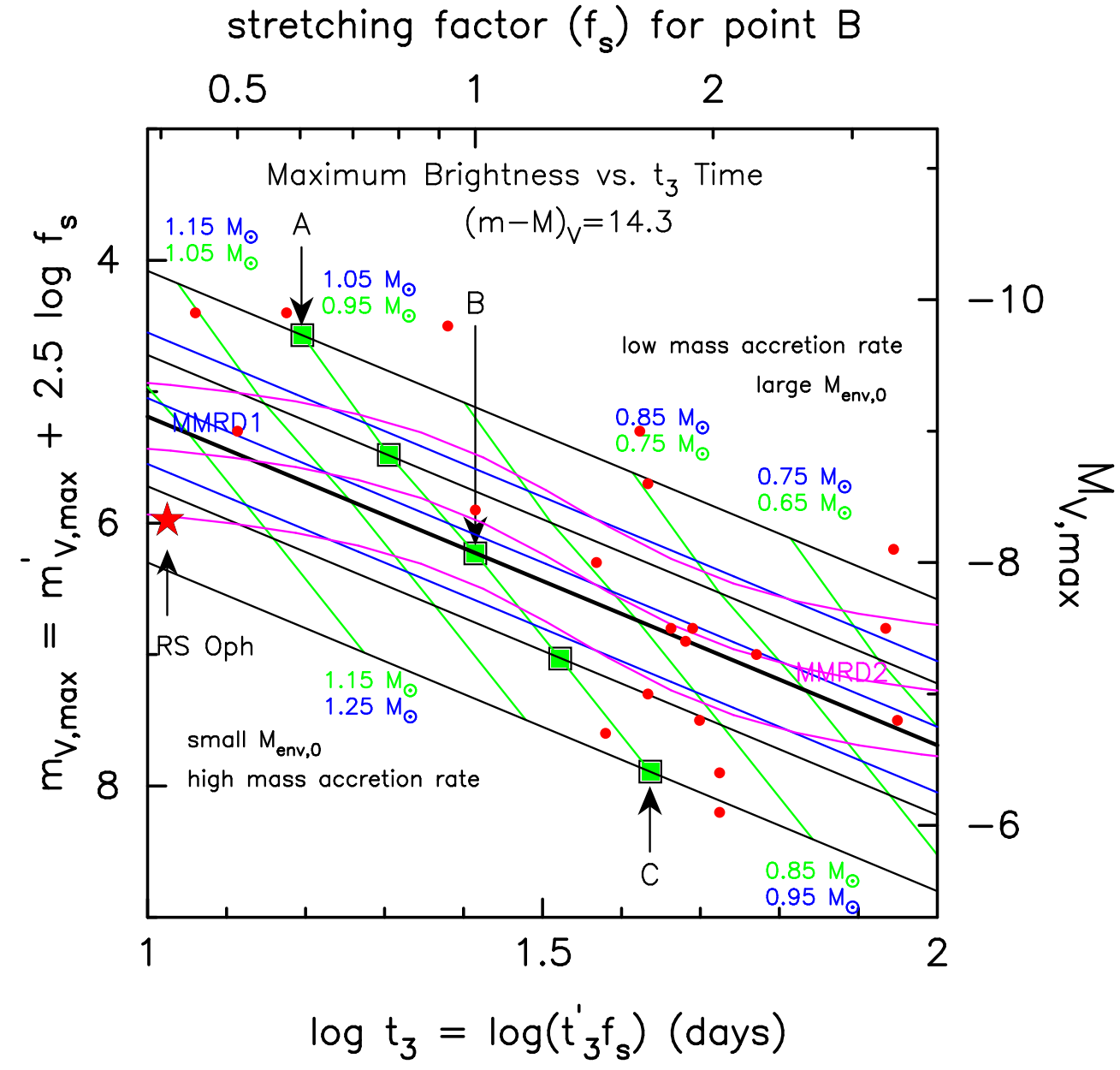

FIG. 15.- The maximum magnitude-rate of decline (MMRD) relation against $t_{3}$ time based on the universal decline law. Point B represents a nova on a $0.95 M_{\odot}$ WD with a typical initial envelope mass. The black solid line passing through point B is corresponding to an MMRD relation of novae having a typical initial envelope mass but different WD mass. The black solid line passing through point A is an MMRD relation for a much larger initial envelope mass than that of point $\mathrm{B}$ (much lower mass accretion rates). The black solid line passing through point $\mathrm{C}$ is an MMRD relation for a much smaller initial envelope mass than that of point B (much higher mass accretion rates). Therefore, the region between upper and lower black solid lines represents our theoretical MMRD relation. Green solid lines connect the same WD mass, from 1.15 to $0.65 M_{\odot}$ by $0.1 M_{\odot}$ step (green attached number) for "CO nova 2 ," but from 1.25 to $0.75 M_{\odot}$ by $0.1 M_{\odot}$ step (blue attached number) for "Ne nova 2." The absolute magnitude $M_{V \text {,max }}$ at maximum is obtained from calibration with V1668 Cyg of the distance modulus $(m-M)_{V}=14.3$. Red filled star marks: the recurrent nova RS Oph as an example for a very high mass accretion rate and very short timescale $t_{3}$ (very small $f_{\mathrm{s}}$ ). Two well-known MMRD relations are added: Kaler-Schmidt's law (Schmidt 1957) of Equation 38 labeled "MMRD1" (blue solid lines), and Della Valle \& Livio's (1995) law of Equation (39) labeled "MMRD2" (magenta solid lines). We add upper/lower bounds of \pm 0.5 mag for each of these two MMRD relations. Red filled circles: We also add MMRD points for individual novae taken from Table 5 of Downes \& Duerbeck (2000).

a line passing through point B in Figure 15. This is our theoretical MMRD relation, the parameter of which is $M_{\mathrm{WD}}$ (or $f_{\mathrm{s}}$ ). We also show the equivalent parameter, stretching factor $f_{\mathrm{s}}$ (against point $\mathrm{B}$ ), in the upper axis of the same figure.

The black thick solid line passing through point B shows quite a good agreement with the central blue and magenta lines, which are empirical MMRD relations obtained from observation as will be explained bellow.

\subsection{Scatter of MMRD relations from average}

If the initial envelope mass $M_{\mathrm{env}, 0}$ is larger or smaller than that of point B (which is an average value, see Figure 2), we obtain different MMRD relations. In Figure 15, we show five points of green filled squares corresponding to different initial envelope mass of $M_{\mathrm{env}, 0}$. Point A ( $t_{3}^{\prime}=16$ days, $\left.m_{V, \max }^{\prime}=4.6\right)$ corresponds to a larger envelope mass at ignition (point $\mathrm{A}$ in Figure 2). The black solid line passing through point $A$ is other $m_{V, \max }-\log t_{3}$ relation for a much larger initial envelope mass of $M_{\text {env }, 0}$ (at point A in Figure 2), i.e.,

$$
M_{V, \text { max }}=2.5 \log t_{3}+4.6-2.5 \log 16-14.3=2.5 \log t_{3}-12.7 \text {. }
$$

On the other hand, the black solid line passing through point $\mathrm{C}\left(t_{3}^{\prime}=43\right.$ days, $\left.m_{V, \text { max }}^{\prime}=7.9\right)$ is also different $m_{V, \max }-\log t_{3}$ relation for a much smaller initial envelope mass (at point $\mathrm{C}$ in Figure 2), i.e.,

$$
M_{V, \text { max }}=2.5 \log t_{3}+7.9-2.5 \log 43-14.3=2.5 \log t_{3}-10.5 \text {. }
$$

For a given WD mass, $M_{\mathrm{env}, 0}$ is larger (smaller) for a smaller (larger) accretion rate. Points A, B, and C correspond to different ignition masses for a given WD mass. When the accretion rate is much smaller than that for point $\mathrm{B}$, the brightness reaches, for example, point $A$ at maximum and then we have a shorter $t_{3}$ time (see Figure 2). In the case of a larger accretion rate than that for point $\mathrm{B}$, the brightness goes up only to point $\mathrm{C}$ at maximum and we have a longer $t_{3}$ time. So, even for a given $M_{\mathrm{WD}}$ and chemical composition of the envelope, we have different $t_{3}$ time, depending on $M_{\mathrm{env}, 0}$. In other words, a 
variation of mass accretion rates from an average value makes a divergence (or width) in the MMRD relation for individual novae.

The five points including points $\mathrm{A}, \mathrm{B}$, and $\mathrm{C}$ are calculated for V1668 Cyg model light curves, all of which correspond to the $0.95 M_{\odot}$ WD of "CO nova 2" $\left(f_{\mathrm{s}}=1\right)$. The green solid line connecting these five points represents the $M_{V, \max }-\log t_{3}$ relation for $0.95 M_{\odot}$ WD of "CO nova 2." We then obtain other $M_{V, \max }-\log t_{3}$ lines for other WD masses as shown by green lines (attached numbers represent WD masses) in Figure 15 In the case of neon novae, we also obtain similar lines, which are also shown in Figure 15. These lines are almost overlapped to those of "CO nova 2" but different WD masses (blue attached numbers for WD masses).

\subsection{Comparison with other MMRD relations}

Our MMRD $\left(M_{V, \max }-\log t_{3}\right)$ relation passing through point $\mathrm{B}$ is in a good agreement with two well-known MMRD relations empirically obtained: one is Kaler-Schmidt's law (Schmidt 1957), i.e.,

$$
M_{V, \text { max }}=-11.75+2.5 \log t_{3}
$$

and the other is Della Valle \& Livio's (1995) law, i.e.,

$$
M_{V, \max }=-7.92-0.81 \arctan \left(\frac{1.32-\log t_{2}}{0.23}\right) \text {, }
$$

where $M_{V, \max }$ is the absolute $V$ magnitude at maximum and we use Equation (29) to calculate $t_{2}$ from $t_{3}$ in Equation (39) in Figure 15. Kaler-Schmidt's law is denoted by a blue solid line with two attendant blue solid lines corresponding to \pm 0.5 mag brighter/darker cases. Della Valle \& Livio's law is indicated by magenta solid lines. These two well-known empirical MMRD relations are very close to our theoretical MMRD relation at average.

We also add observational MMRD points $\left(M_{V, \max }\right.$ and $t_{3}$ ) for individual novae, data of which are taken from Table 5 of Downes \& Duerbeck (2000), although their $t_{3}$ are not intrinsic but observational face values. It is clearly shown that a large scatter of individual points (red filled circles) from the two empirical MMRD relations falls into between upper/lower cases of our MMRD relations for the largest/smallest initial envelope mass of $M_{\text {env, } 0}$. This simply means that there is a second parameter to specify the MMRD relation for individual novae. The main parameter is the WD mass represented by stretching factor $f_{\mathrm{s}}$. The second parameter is the initial envelope mass (or the mass accretion rate to the WD). This second parameter can reasonably explain the scatter of individual novae from the empirical MMRD relations ever proposed.

It is interesting to place the position of the recurrent nova RS Oph in this diagram. RS Oph locates in the smallest corner of $t_{3}$ time and a relatively darker level of $M_{V \text {,max }}$, near the $M_{V, \max }-\log t_{3}$ relation passing through point $\mathrm{C}$. This indicates a much smaller envelope mass at the optical maximum, suggesting that the mass accretion rate to the WD is very high. This situation is very consistent with the total picture of recurrent novae; a very massive WD close to the Chandrasekhar mass and a high mass accretion rate to the WD (e.g., Hachisu \& Kato 2001). In this figure, we adopt $M_{V, \max }=-8.3$ and $t_{3}=10.5$ days with the distance of $d=1.6$ kpc (Hjellming et al. 1986), absorption of $A_{V}=3.1 E(B-V)=$ $3.1 \times 0.73=2.3($ Snijders $1987 \mathrm{a} b), m_{V, \max }=5.0($ Rosino 1987), and $t_{3}=10.5$ days from optical light curve fitting

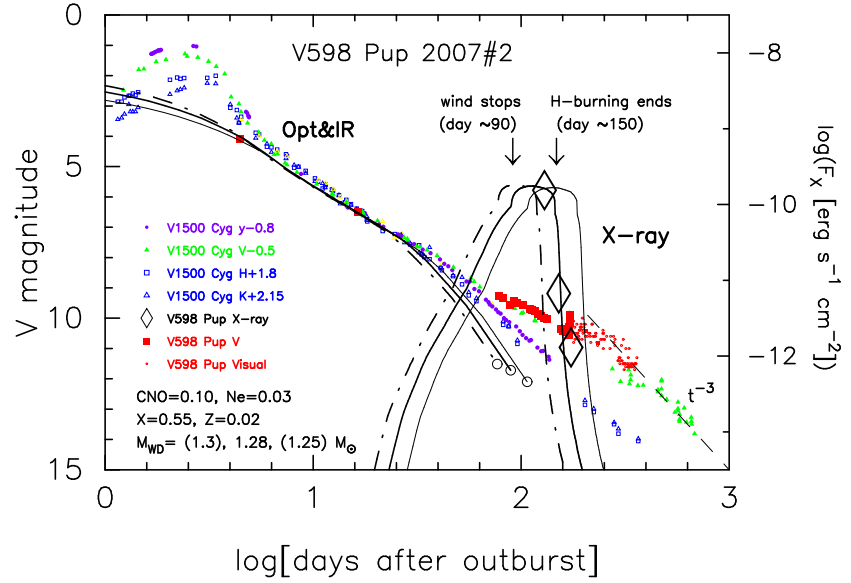

FIG. 16.- Optical and supersoft X-ray light curves for V598 Pup. We plot free-free model light curves (labeled "Opt\&IR") and $0.2-0.6 \mathrm{keV}$ supersoft X-ray fluxes (labeled "X-ray") of three WD mass models of $M_{\mathrm{WD}}=1.3 M_{\odot}$ (dash-dotted line), 1.28 $M_{\odot}$ (thick solid line), and $1.25 M_{\odot}$ (thin solid line), for the envelope chemical composition of "Ne nova 2." We select a best model of $1.28 M_{\odot}$ WD among three WD mass models, in order to reproduce the supersoft X-ray data. The supersoft X-ray data is sandwiched between the 1.3 and $1.25 M_{\odot}$ WDs, so we safely regard that $M_{\mathrm{WD}}=1.28 \pm 0.03 M_{\odot}$. The X-ray flux data (large diamonds) without error bars are taken from Read et al. (2007, 2008). Optical $V$ data of V598 Pup are taken from Poimanski et al. (2007) and visual data are from the American Association of Variable Star Observers (AAVSO). Arrows indicate two epochs when the optically thick wind stops about 90 days after the outburst and when the steady hydrogen shell burning atop the WD ends about 150 days after the outburst for our $1.28 M_{\odot}$ WD model. We added optical and near IR data of V1500 Cyg for comparison (see Figure 9].

with our free-free model light curves (Hachisu \& Kato 2001; Hachisu et al. 2006, 2007).

\section{LIGHT CURVE ANALYSIS OF TEN NOVAE WITH SUPERSOFT} X-RAY EMISSION

In this section, we examine ten classical novae which we have not yet analyzed in our series of papers, i.e., V598 Pup, V382 Vel, V4743 Sgr, V1281 Sco, V597 Pup, V1494 Aql, V2467 Cyg, V5116 Sgr, V574 Pup, and V458 Vul, in the order of increasing timescale. All are supersoft X-ray detected novae, so we can check whether or not our prediction formulae of a supersoft X-ray phase work on these novae.

\subsection{V598 Pup 2007\#2}

V598 Puppis (Nova Puppis 2007 No.2) was serendipitously discovered by the XMM-Newton slew survey (Read et al. 2008). Torres et al. (2007) subsequently found an optically bright counter part of this X-ray object. The spectra obtained on November 16.34 UT (day 170) reveal numerous features suggestive of a nova that already entered the auroral phase (see also Read et al. 2008). Analysis of All Sky Automated Survey (ASAS) archive showed that the $V$ magnitude rose to $m_{V}=4.1$ from $m_{V} \gtrsim 14$ between June 1.966 UT and June 5.968 UT (Poimanski et al. 2007). Since we cannot specify the exact date of the outburst, we assume here that the outburst day is $t_{\mathrm{OB}}=\mathrm{JD} 2454253.0$ (2007 June 1.5 UT).

The X-ray satellite Swift detected X-ray flux from V598 Pup, which declined rapidly from $F_{\mathrm{X}}(0.2-2 \mathrm{keV})=1.5 \times$ $10^{-10}$ (day $\sim 130$ ) to $6.7 \times 10^{-12}$ (day $\sim 150$ ) and then to $1.3 \times 10^{-12} \mathrm{erg} \mathrm{s}^{-1} \mathrm{~cm}^{-2}$ (at day $\sim 170$ after the outburst) (Read et al. 2007) as shown in Figure 16. This quick drop in the X-ray flux clearly showed that hydrogen burning on the WD ended around 150 days after the outburst.

Figure 16 shows observed optical light curves of V598 Pup as well as the X-ray data. Unfortunately there are only two 
TABLE 6

Timescales of Novae Estimated from Universal DeCline LaW

\begin{tabular}{|c|c|c|c|c|c|c|c|c|c|}
\hline object & $\cdots$ & $\begin{array}{c}t_{2}{ }^{\mathrm{a}} \\
\text { (day) }\end{array}$ & $\begin{array}{c}t_{3}{ }^{\mathrm{a}} \\
\text { (day) }\end{array}$ & $\begin{array}{l}t_{\text {break }} \\
\text { (day) }\end{array}$ & $\begin{array}{l}t_{\text {wind }} \\
\text { (day) }\end{array}$ & $\begin{array}{c}t_{\mathrm{H}-\text { burning }} \\
\text { (day) }\end{array}$ & $\begin{array}{c}t_{\text {emerge }} \\
\text { (day) }\end{array}$ & $\begin{array}{l}M_{\mathrm{WD}} \\
\left(M_{\odot}\right)\end{array}$ & chem.comp. $^{b}$ \\
\hline V598 Pup 2007\#2 & $\ldots$ & - & - & 40 & 90 & 150 & - & 1.28 & Ne nova 2 \\
\hline V382 Vel 1999 & $\ldots$ & 6.6 & 12.4 & 52 & 120 & 220 & 32 & 1.23 & Ne nova 2 \\
\hline V4743 Sgr 2002\#3 & $\ldots$ & 9.4 & 17 & 70 & 180 & 380 & 36 & 1.15 & Ne nova 2 \\
\hline V1281 Sco $2007 \# 2$ & $\ldots$ & 9.5 & 16.7 & 78 & 190 & 425 & - & 1.13 & Ne nova 2 \\
\hline V597 Pup 2007\#1 & $\ldots$ & 8.7 & 16.5 & 74 & 200 & 430 & 52 & 1.2 & Ne nova 3 \\
\hline V1494 Aql 1999\#2 & $\ldots$ & 11.1 & 21.2 & 96 & 260 & 670 & 66 & 1.13 & Ne nova 3 \\
\hline V1494 Aql 1999\#2 & $\cdots$ & 11.7 & 22.1 & 102 & 260 & 670 & 72 & 1.06 & Ne nova 2 \\
\hline V1494 Aq1 1999\#2 & $\ldots$ & 14.7 & 26.6 & 120 & 285 & 675 & 95 & 0.92 & CO nova 2 \\
\hline V2467 Cyg 2007 & $\ldots$ & 14.3 & 25.3 & 110 & 290 & 765 & 68 & 1.11 & Ne nova 3 \\
\hline V2467 Cyg 2007 & $\ldots$ & 14.8 & 27.9 & 113 & 290 & 765 & 75 & 1.04 & Ne nova 2 \\
\hline V2467 Cyg 2007 & $\ldots$ & 17.6 & 31.7 & 135 & 310 & 750 & 96 & 0.90 & CO nova 2 \\
\hline V5116 Sgr 2005\#2 & $\ldots$ & 16.7 & 30.4 & 127 & 355 & 1000 & 91 & 1.07 & Ne nova 3 \\
\hline V5116 Sgr 2005\#2 & $\cdots$ & 16.2 & 30.5 & 134 & 350 & 995 & 100 & 1.0 & Ne nova 2 \\
\hline V5116 Sgr 2005\#2 & $\ldots$ & 20.0 & 35.8 & 169 & 390 & 1010 & 130 & 0.85 & CO nova 2 \\
\hline V574 Pup 2004 & $\ldots$ & 18.6 & 35.0 & 144 & 390 & 1140 & - & 1.05 & Ne nova 3 \\
\hline V458 Vul 2007 & $\cdots$ & 20.4 & 37.2 & 145 & 415 & 1250 & 62 & 0.93 & CO nova 4 \\
\hline V1974 Cyg 1992 & $\ldots$ & 16 & 26 & 108 & 250 & 600 & 90 & 1.05 & Ne nova 2 \\
\hline V1668 Cyg 1978 & $\ldots$ & 14.4 & 26 & 100 & 280 & 720 & 87 & 0.95 & CO nova 3 \\
\hline V1500 Cyg 1975 & $\ldots$ & 7.2 & 13 & 70 & 180 & 380 & 50 & 1.15 & Ne nova 2 \\
\hline
\end{tabular}

${ }^{\mathrm{a}} t_{2}$ and $t_{3}$ times are all intrinsic values estimated along our theoretical light curve

${ }^{\mathrm{b}}$ see Table1

TABLE 7

SumMary of Physical Properties of NovaE

\begin{tabular}{|c|c|c|c|c|c|c|c|c|c|}
\hline object & $\ldots$ & $\begin{array}{l}M_{\mathrm{WD}} \\
\left(M_{\odot}\right)\end{array}$ & $\begin{array}{c}M_{2} \\
\left(M_{\odot}\right)\end{array}$ & $\begin{array}{l}P_{\text {orb }} \\
\text { (day) }\end{array}$ & $\begin{array}{c}a \\
\left(R_{\odot}\right)\end{array}$ & $\begin{array}{c}R_{1}^{*} \\
\left(R_{\odot}\right)\end{array}$ & $\begin{array}{c}M_{\text {wind }}{ }^{a} \\
\left(10^{-5} M_{\odot}\right)\end{array}$ & $\begin{array}{l}M_{\text {env }, 0}{ }^{\mathrm{b}} \\
\left(10^{-5} M_{\odot}\right)\end{array}$ & chem.comp. ${ }^{\mathrm{c}}$ \\
\hline V598 Pup 2007\#2 & $\ldots$ & 1.28 & - & - & - & - & 0.31 & 0.34 & Ne nova 2 \\
\hline V382 Vel 1999 & $\ldots$ & 1.23 & 0.31 & 0.1461 & 1.35 & 0.68 & 0.43 & 0.48 & Ne nova 2 \\
\hline V4743 Sgr 2002\#3 & $\cdots$ & 1.15 & 0.70 & 0.2799 & 2.2 & 0.93 & 0.69 & 0.78 & Ne nova 2 \\
\hline V1281 Sco 2007\#2 & $\begin{array}{l}\cdots \\
\cdots\end{array}$ & 1.13 & - & - & - & - & 0.92 & 1.02 & Ne nova 2 \\
\hline V597 Pup 2007\#1 & $\ldots$ & 1.2 & 0.22 & 0.1112 & 1.1 & 0.58 & 1.06 & 1.14 & Ne nova 3 \\
\hline V1494 Aql 1999\#2 & $\begin{array}{l}\cdots \\
\ldots\end{array}$ & 1.13 & 0.28 & 0.1346 & 1.24 & 0.62 & 1.04 & 1.12 & Ne nova 3 \\
\hline V1494 Aq1 1999\#2 & $\ldots$ & 1.06 & 0.28 & 0.1346 & 1.22 & 0.60 & 1.11 & 1.26 & Ne nova 2 \\
\hline V1494 Aql 1999\#2 & $\ldots$ & 0.92 & 0.28 & 0.1346 & 1.17 & 0.57 & 1.75 & 2.00 & CO nova 2 \\
\hline V2467 Cyg 2007 & $\ldots$ & 1.11 & 0.35 & 0.1596 & 1.40 & 0.67 & 1.08 & 1.23 & Ne nova 3 \\
\hline V2467 Cyg 2007 & $\ldots$ & 1.04 & 0.35 & 0.1596 & 1.38 & 0.65 & 1.16 & 1.34 & Ne nova 2 \\
\hline V2467 Cyg 2007 & $\begin{array}{l}\cdots \\
\ldots\end{array}$ & 0.90 & 0.35 & 0.1596 & 1.33 & 0.62 & 1.67 & 1.90 & CO nova 2 \\
\hline V5116 Sgr 2005\#2 & $\ldots$ & 1.07 & 0.25 & 0.1238 & 1.15 & 0.58 & 1.25 & 1.43 & Ne nova 3 \\
\hline V5116 Sgr $2005 \# 2$ & $\ldots$ & 1.00 & 0.25 & 0.1238 & 1.13 & 0.56 & 1.30 & 1.53 & Ne nova 2 \\
\hline V5116 Sgr 2005\#2 & $\ldots$ & 0.85 & 0.25 & 0.1238 & 1.08 & 0.53 & 2.17 & 2.52 & CO nova 2 \\
\hline V574 Pup 2004 & $\ldots$ & 1.05 & - & - & - & - & 1.25 & 1.46 & Ne nova 3 \\
\hline V458 Vul 2007 & $\ldots$ & 0.93 & $0.74^{\mathrm{d}}$ & 0.5895 & 3.51 & 1.40 & 1.26 & 1.53 & CO nova 4 \\
\hline V1974 Cyg 1992 & $\ldots$ & 1.05 & 0.15 & 0.0813 & 0.85 & 0.44 & 1.0 & 1.17 & Ne nova 2 \\
\hline V1668 Cyg 1978 & $\begin{array}{l}\cdots \\
\cdots\end{array}$ & 0.95 & 0.29 & 0.1384 & 1.21 & 0.58 & 1.1 & 1.3 & CO nova 3 \\
\hline V1500 Cyg 1975 & $\ldots$ & 1.15 & 0.29 & 0.1396 & 1.28 & 0.64 & 0.71 & 0.8 & Ne nova 2 \\
\hline
\end{tabular}

observational points available before 77 days after the outburst. To understand the characteristic properties of V598 Pup light curves, we add observational points of V1500 Cyg that show a very similar light curve in the later phase. We can see that two points of $V$ mag in the early phase are just on our free-free model light curve but the $V$ data after day $\sim 70$ are those in the nebular phase and already deviated from the expected free-free light curve. This deviation often occurs in novae when $V$ magnitude is contaminated by strong emission lines such as [O III] in the nebular phase (see Read et al. 2008, for emission lines of V598 Pup), as already discussed in Hachisu \& Kato (2006, 2007) and Hachisu et al. (2008). Thus we regard that the light curve of V598 Pup decays very similarly to that of V1500 Cyg, which follows our universal decline law from day $\sim 5$ to day $\sim 70$.

No abundance determinations are available for V598 Pup so far. We here assume two typical cases, one is for oxygenneon novae of "Ne nova 2"and the other is for carbon-oxygen novae of "CO nova 2" as listed in Table 1 .

The emergence/decay times of supersoft X-ray are a good indicator of the WD mass (Hachisu \& Kato 2006; Hachisu et al. 2008). We have calculated several models for different WD masses with these two chemical compositions. Among the models of $M_{\mathrm{WD}}=1.3,1.28$, and $1.25 M_{\odot}$, we have selected $M_{\mathrm{WD}}=1.28 M_{\odot}$ as a best-reproducing model for "Ne nova 2," but, $M_{\mathrm{WD}}=1.2 M_{\odot}$ for "CO nova 2 " as a 
best-reproducing one among $M_{\mathrm{WD}}=1.25,1.2$, and $1.15 M_{\odot}$, which are not shown in the figure. The best models are selected by careful eye fitting with the supersoft X-ray data because there are only three points. The optical fitting cannot be used for the selection because all of three model light curves go through the two observational $V$ points as seen in Figure 16. We define the "error" in the WD mass determination $\left( \pm 0.03 M_{\odot}\right)$ from larger and smaller WD masses, $M_{\mathrm{WD}}=1.3$ and $1.25 M_{\odot}$, safely cover the decay phase of supersoft X-ray flux for the fixed chemical composition.

The estimated WD mass of $1.2 M_{\odot}$ for "CO nova 2" exceeds the upper limit mass for CO WDs born in a binary, i.e., $M_{\mathrm{CO}} \lesssim 1.07 M_{\odot}$ (Umeda et al. 1999), so we regard that the WD of V598 Pup is very likely an ONeMg WD. Therefore, we adopt the $1.28 M_{\odot}$ WD of "Ne nova 2 " as a best model, which has $t_{\text {break }}=40, t_{\text {wind }}=90$, and $t_{\mathrm{H}-\text { burning }}=150$ days after the outburst (see Figure 16).

We have also calculated the ejecta mass of $M_{\text {wind }} \sim 3 \times$ $10^{-6} M_{\odot}$, which is lost by the optically thick wind (see, e.g., Kato \& Hachisu 1994; Hachisu \& Kato 2006, for more detail of ejecta mass calculation). However, it should be noted that $M_{\text {wind }}$ could be the subject to significant uncertainty because we missed the real optical maximum. Various physical parameters of novae thus obtained are summarized in Tables 6] and 7

We are not able to determine $t_{2}$ and $t_{3}$ from the light curve because of lack of visual magnitudes around the optical peak.

The mass determination of a WD depends weakly on the chemical composition of the envelope, especially, on the hydrogen content $X$ (Hachisu \& Kato 2006, 2007; Hachisu et al. 2008; Hachisu \& Kato 2009). We have estimated this dependency from two other chemical composition models, i.e., "Ne nova 1" and "Ne nova 3" in Table 1. We similarly obtain a best-reproducing model of $M_{\mathrm{WD}}=1.2 M_{\odot}$ for $X=0.35$ ("Ne nova 1") and $M_{\mathrm{WD}}=1.32 M_{\odot}$ for $X=0.65$ ("Ne nova 3"). The resultant dependency can be approximated as

$$
M_{\mathrm{WD}}(X) \approx\{1.28+0.4(X-0.55)\} M_{\odot},
$$

for V598 Pup. Here, we have already obtained $M_{\mathrm{WD}}(X=$ $0.55)=1.28 M_{\odot}$. Therefore, we may conclude that the WD mass is $M_{\mathrm{WD}}=1.28 \pm 0.04 M_{\odot}$ for a typical hydrogen content between $X=0.45-0.65$ for V598 Pup. The WD mass estimate depends also on the $\mathrm{CNO}$ abundance, but the difference among various $X_{\mathrm{CNO}}$ models in Table 1 is smaller than the difference among various hydrogen content $(X)$ models (see Hachisu \& Kato 2006, for more detail of $X$ dependency).

The distance to V598 Pup can be estimated by two methods even if the optical maximum is not available. The first one is to use the absolute magnitude of our free-free model light curves in Table 3 . We use our best-reproducing model in Figure 16 and measure the brightness at the end of the freefree (FF) light curve, $m_{\mathrm{w}}$, and obtain the distance modulus to V598 Pup as

$$
\left[(m-M)_{V}\right]_{\mathrm{FF}}=m_{\mathrm{w}}-M_{\mathrm{w}}=11.8-0.1=11.7 .
$$

Here we adopt $m_{\mathrm{w}}=11.8$ from Figure 16 and use the absolute magnitude of $M_{\mathrm{w}}=0.1$ at the end point of $1.28 M_{\odot}$ WD model light curve, which is calculated by interpolation between the $1.25 M_{\odot}$ and $1.3 M_{\odot}$ WD models in Table 3 We call this "FF method" as introduced in Section 3.6 It should be addressed that the FF method is rather robust and gives $(m-M)_{V}=11.6-0.0=11.6$ for $1.3 M_{\odot} \mathrm{WD}$ or $(m-M)_{V}=12.2-0.4=11.8$ for $1.25 M_{\odot}$ WD even if we miss a best model. We also emphasize that this FF method does not require the optical maximum like the MMRD relations, so it can be used even if the optical maximum is not available like V598 Pup.

The next method is to use direct comparison with the other nova light curve the distance modulus of which is already known. We have already done this method in Section 3.6 (see Figures 7, 10, and 11). The direct comparison between V1500 Cyg and V598 Pup in Figure 16 gives the difference of distance moduli of these two novae, i.e.,

$$
\left[5 \log (d / 10)+A_{V}\right]_{\mathrm{V} 1500 \mathrm{Cyg}}-\left[5 \log (d / 10)+A_{V}\right]_{\mathrm{V} 598 \text { Pup }}=0.5 \text {. }
$$

When we adopt $(m-M)_{V}=12.5$ from Equation (20) for V1500 Cyg, we obtain

$$
\left[(m-M)_{V}\right]_{\mathrm{LC}}=\left[5 \log (d / 10)+A_{V}\right]_{\mathrm{V} 598 \text { Pup }}=12.0,
$$

where LC means the direct light curve (LC) fitting. It should be again addressed that the LC method does not need the optical maximum like the MMRD relations, so it can be used even if we missed the optical maximum like V598 Pup.

The difference of $0.3 \mathrm{mag}$ in the distance modulus estimation can be understood as follows. These two nova light curves look very similar in the later phase but the fitting mass is different between $1.28 M_{\odot}$ (V598 Pup) and $1.15 M_{\odot}$ (V1500 Cyg). The absolute magnitude at 20 days, for example, is about 0.4 mag brighter in the $1.15 M_{\odot}$ WD model than in the $1.25 M_{\odot}$ as shown in Figure 7 We have experienced that the same thing happened when we use fitting only of optical light curves as shown in Figure 8 . In this figure, we obtain \pm 0.4 mag larger $(m-M)_{V}$ when the fitting mass is missed by $\mp 0.1 M_{\odot}$, respectively. In this sense, the LC method may have an error of 0.4 mag or so even if two nova light curves look very similar in the later phase.

The above two estimates are listed in Table 8 , being roughly consistent with each other, i.e., $(m-M)_{V}=11.9 \pm 0.2$ (averaged with equal weight). These two distance moduli give the distance of $d=2.1 \pm 0.2 \mathrm{kpc}$ for $A_{V}=0.27$ (Read et al. 2008). This distance is consistent with various estimates discussed by Read et al. (2008).

\subsection{V382 Vel 1999}

V382 Velorum (Nova Velorum 1999) was discovered independently by Williams and Gilmore Williams \& Gilmore 1999). The nova reached $m_{V}=2.7$ at maximum on 1999 May 23 and then decayed to $m_{V}=16.6$ (Woudt et al. 2005) as shown in Figure 17. V382 Vel is a fast nova and identified as a neon nova (Woodward et al. 1999). We here adopt a set of chemical composition of a typical neon nova, "Ne nova 2" in Table 1, based on the composition analyses by Shore et al. (2003) and by Augusto \& Diaz (2003) (see Table 1 of Hachisu \& Kato 2006). We also assume that the outburst day is $t_{\mathrm{OB}}=\mathrm{JD} 2451319.0$ (1999 May 20.5 UT) from information of IAU Circulars 7176 and 7184, i.e., $m_{\mathrm{vis}}>12$ on May $20.57 \mathrm{UT}, m_{\mathrm{vis}}=4.3$ on May $21.62 \mathrm{UT}, m_{\mathrm{vis}}=3.6$ on May 21.725 UT, and $m_{V}=3.1$ on May 22.396 UT.

Supersoft X-rays were detected by BeppoSAX about six months after the outburst (Orio et al. 2002). After that, the flux decayed rapidly as shown in Figure 17. The Chandra observations by Burwitz et al. (2002) and by Ness et al. (2005) suggested that the hydrogen burning ended around 220 days after the outburst. The theoretical supersoft X-ray phase is very sensitive to the WD mass whereas it depends weakly 


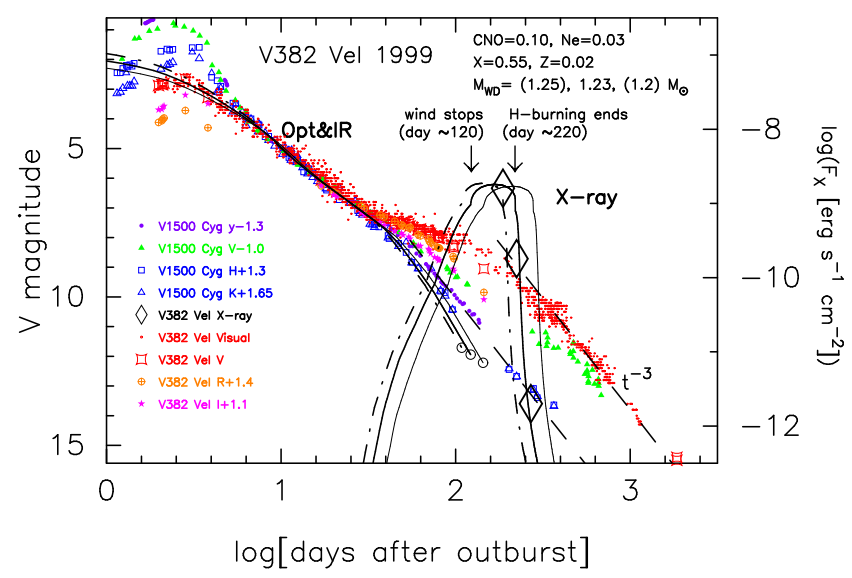

FIG. 17.- Same as Figure 16 but for V382 Vel. Observational data of optical $V$ (boxes with sharp corners) and $R$ (circles with a plus), near infrared I (star marks), and supersoft X-ray (large diamonds). We plot three model light curves of $M_{\mathrm{WD}}=1.25$ (thick dash-dotted lines), 1.23 (thick solid lines), and $1.2 M_{\odot}$ (thin solid lines) WDs for the envelope chemical composition of "Ne nova 2." We select the $1.23 M_{\odot}$ WD as a best model among three of $1.25,1.23$, and $1.2 M_{\odot}$ WDs and can reproduce the supersoft X-ray data (three points). The supersoft X-ray data is sandwiched between the 1.25 and $1.2 M_{\odot}$ WDs, so we safely regard that $M_{\mathrm{WD}}=1.23 \pm 0.03 M_{\odot}$. Observational V382 Vel data of $V, R$, and $I$ magnitudes are taken from IAU Circulars 7176, 7179, 7196, 7209, 7216, 7226, 7232, 7238, and 7277. The darkest observational $V$ magnitudes at day $\sim 1850$ are taken from Woudt et al. (2005). Visual magnitudes (small circles) are from AAVSO. The X-ray absorbed flux data (large open diamonds) of V382 Vel are taken from Orio et al. (2002) and Burwitz et al. (2002) without error bars.

on the chemical composition of the envelope. Our X-ray light curve fitting suggests that 1 ) the WD mass is as massive as $M_{\mathrm{WD}}=1.23 \pm 0.03 M_{\odot}$ and 2) the supersoft X-ray phase started from day $\sim 120$. We have also estimated the ejecta mass of $M_{\text {wind }} \sim 4.3 \times 10^{-6} M_{\odot}$ (Table 7) for the $1.23 M_{\odot}$ WD model. Della Valle et al. (2002) derived the ejecta mass of $M_{\mathrm{ej}} \sim 6 \times 10^{-6} M_{\odot}$ from the flux of $\mathrm{H} \alpha$ emission, which is consistent with our estimate.

Our model of $1.23 M_{\odot}$ WD shows $t_{\text {break }}=52, t_{\text {wind }}=120$, and $t_{\mathrm{H}-\text { burning }}=220$ days after the outburst (see Figure 17). Since the $V$ magnitude reached its maximum $t_{0}=2.6$ days after the outburst, we obtain "intrinsic" $t_{2}=6.6$ and $t_{3}=12.4$ days along our model light curve. For this nova, since the observed data decays almost along our model light curves, the "face" values of $t_{2}$ and $t_{3}$ are practically the same as those of our "intrinsic" $t_{2}$ and $t_{3}$. It should be noted here that the assumed epoch of $t_{\mathrm{OB}}=\mathrm{JD} 2451319.0$ (1999 May 20.5 UT) hardly affect our estimation of $t_{2}$ and $t_{3}$ times because we do not use the value at $t_{\mathrm{OB}}$. Usually we move the model light curve "back and forth" and "up and down" against the observed value as shown in Figure 8 and this process simply means that we fit our model light curve with the decay phase after maximum.

In Figure 17, we add $V$ and $y$ light curves of V1500 Cyg for comparison. From the difference between $V$ and $y$ bands, we can roughly evaluate the influence of strong emission lines that contribute extra flux to $V$ magnitude above free-free continuum flux. Comparing the light curves of V1500 Cyg, we regard that V382 Vel light curve is contaminated by strong emission lines from $\sim 40$ days after the outburst; all the $V, R$, and $I$ magnitudes begins to diverge from each other and start to deviate from our universal decline law (thick solid line).

The WD mass estimate depends weakly on the chemical composition. Changing hydrogen content $X$, we obtain a bestreproducing model of $M_{\mathrm{WD}}=1.13 M_{\odot}$ for $X=0.35$ ("Ne nova
1 ") and $M_{\mathrm{WD}}=1.28 M_{\odot}$ for $X=0.65$ ("Ne nova 3"). Thus, we obtain the dependency of the WD mass on $X$, i.e.,

$$
M_{\mathrm{WD}}(X) \approx\{1.23+0.5(X-0.55)\} M_{\odot},
$$

for V382 Vel. This relation on the WD mass is a bit steeper than that for V598 Pup. We may also conclude that the WD mass is $M_{\mathrm{WD}}=1.23 \pm 0.05 M_{\odot}$ for a typical hydrogen content between $X=0.45-0.65$ for V382 Vel, which corresponds to the diversity in the composition determination between $X=0.66$ (Shore et al. 2003) and $X=0.47$ (Augusto \& Diaz 2003).

The distance modulus to V382 Vel can be calculated in the same two ways as those in the previous subsection (V598 Pup). First, the FF method gives

$$
\left[(m-M)_{V}\right]_{\mathrm{FF}}=m_{\mathrm{w}}-M_{\mathrm{w}}=11.9-0.5=11.4,
$$

for the $1.23 M_{\odot}$ WD model. Even if we use two other models of $1.25 M_{\odot}$ and 1.2 $M_{\odot}$ WDs in Figure 17 , this method gives a similar result of $(m-M)_{V}=11.7-0.4=11.3$ for $1.25 M_{\odot}$, or $(m-M)_{V}=12.2-0.7=11.5$ for $1.2 M_{\odot}$ WD.

Second, the LC method also gives us the absolute brightness, i.e.,

$$
\left[5 \log (d / 10)+A_{V}\right]_{\mathrm{V} 1500 \mathrm{Cyg}}-\left[5 \log (d / 10)+A_{V}\right]_{\mathrm{V} 382 \mathrm{Vel}}=1.0 .
$$

With $(m-M)_{V}=12.5$ from Equation (20) for V1500 Cyg, the above Equation (46) yields

$$
\left[(m-M)_{V}\right]_{\mathrm{LC}}=\left[5 \log (d / 10)+A_{V}\right]_{\mathrm{V} 382 \mathrm{Vel}}=11.5 .
$$

Kaler-Schmidt's law with $t_{3}=12.4$ days and Della Valle $\&$ Livio's law with $t_{2}=6.6$ days and $m_{V, \max }=2.6$ (Gilmore 1999) give

$$
\left[(m-M)_{V, \max }\right]_{\mathrm{MMRD}}=\left[5 \log (d / 10)+A_{V}\right]_{\mathrm{MMRD}}=11.6(11.4),
$$

where the value outside parenthesis is calculated from KalerSchmidt's law of Equation (38) and the value in parenthesis is calculated from Della Valle \& Livio's law of Equation (39). These two values are consistent with the FF and LC results.

These four values give a distance modulus of $(m-M)_{V}=$ $11.5 \pm 0.1$ for these four different estimations with equal weight and a distance of $d=1.5 \pm 0.1 \mathrm{kpc}$ for $A_{V}=3.1 \times$ $E(B-V)=3.1 \times 0.2=0.62$ (Shore et al. 2003).

These values are consistent with the distance of $1.66 \pm 0.11$ $\mathrm{kpc}$ derived from the maximum magnitude vs. rate of decline (MMRD) relation (Della Valle et al. 2002). On the other hand, Shore et al. (2003) obtained a bit larger distance of $d=2.5 \mathrm{kpc}$, assuming that the UV flux of V382 Vel is equal to that of V1974 Cyg. They took a distance of $3.1 \mathrm{kpc}$ to V1974 Cyg but this value is much larger than a reasonable one of $1.8 \mathrm{kpc}$ (Chochol et al. 1997). If we take the distance of 1.8 $\mathrm{kpc}$ instead of $3.1 \mathrm{kpc}$ to V1974 Cyg, Shore et al.'s method gives a much shorter distance of $1.5 \mathrm{kpc}$ to $\mathrm{V} 382 \mathrm{Vel}$, which is consistent with our estimates.

Finally, we introduce some characteristic features concerning binary nature, that is, the emergence of the companion from the WD photosphere. We define this characteristic time as the emergence time of the companion, i.e., $t_{\text {emerge }}$. The orbital period of $P_{\text {orb }}=0.1461$ days $(3.51 \mathrm{hr})$ was derived by Balman et al. (2006) from the orbital modulations with a full amplitude of 0.02-0.03 mag while a bit longer orbital period of $P_{\mathrm{orb}}=0.1581$ days was obtained by Woudt et al. (2005). So, we estimate the epoch when the companion emerges from 


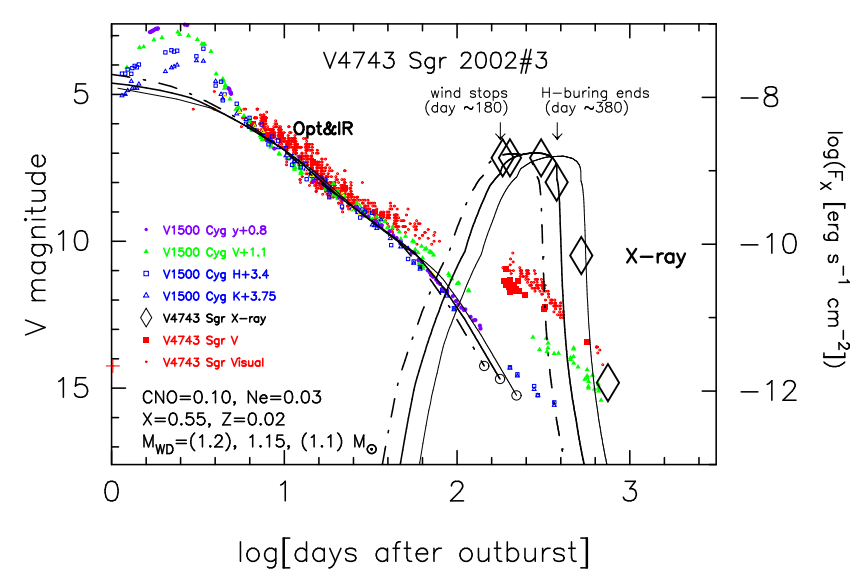

FIG. 18.- Same as Figure 16 but for V4743 Sgr. We plot three model light curves of $M_{\mathrm{WD}}=1.2$ (thick dash-dotted lines), 1.15 (thick solid lines), and $1.1 M_{\odot}$ (thin solid lines) WDs for the envelope chemical composition of "Ne nova 2." We select the $1.15 M_{\odot}$ WD as a best model among three of $1.2,1.15$, and $1.1 M_{\odot}$ WDs and can reproduce the supersoft X-ray data. Visual (small open circles) and $V$ (filled squares) data of V4743 Sgr are taken from IAU Circulars 7975, 7976, and 7982, and from AAVSO. The X-ray flux data (large open diamonds) of V4743 Sgr without error bars are taken from Orio et al. (2003), Orio \& Tepedelenlioglu (2004) and Ness et al. (2007a).

the nova envelope. If the mass of the donor star can be estimated from Warner's (1995) empirical formula, i.e.,

$$
\frac{M_{2}}{M_{\odot}} \approx 0.065\left(\frac{P_{\text {orb }}}{\text { hours }}\right)^{5 / 4} \text {, for } 1.3<\frac{P_{\text {orb }}}{\text { hours }}<9
$$

we have $M_{2}=0.31 M_{\odot}$, which corresponds to the separation of $a=1.35 R_{\odot}$ for $M_{1}=1.23 M_{\odot}$, and the effective Roche lobe radius of the primary component (WD) of $R_{1}^{*}=0.68 R_{\odot}$. It is about $t_{\text {emerge }} \approx 32$ days when the photospheric radius of the nova envelope shrinks to near the orbit (when $R_{\mathrm{ph}} \approx a$ ). This becomes $t_{\text {emerge }} \approx 28$ days if we take the orbit plus the companion's radius (when $R_{\mathrm{ph}} \approx a+R_{2}^{*}$ ).

Hard X-ray $(2-4 \mathrm{keV})$ flux of V382 Vel was detected by ASCA and RXTE (Mukai \& Ishida 2001) about 20 days after the outburst (day 18 with BeppoSAX by Orio et al. $2001 \mathrm{~b}$ ) and reached maximum at day $\sim 30$ and stayed at the same level and then began to decrease at day $\sim 50$. Mukai \& Ishida (2001) interpreted the origin of hard X-rays by a model of internal shocks (Friedjung 1987). This shock may be formed by collision between two ejecta shells as explained by Mukai \& Ishida (2001), but there is another possibility that the shock is formed by collision between nova winds (optically thick winds) and the companion star (see, e.g., Hachisu \& Kato 2005, 2006; Hachisu et al. 2008).

If it is the second case, the hard X-ray emergence should be coincident with the emergence of the companion from the WD photosphere because hard X-ray is probably absorbed deep inside the nova photosphere. The hard $\mathrm{X}$-ray emergence time of $\sim 20$ days is roughly consistent with $t_{\text {emerge }} \approx 30$ days. On the other hand, the hard X-ray flux should be declining as the wind mass-loss rate is decreasing. This corresponds to the break point of free-free emission light curve, because the wind mass-loss rate is rapidly decreasing after the break point of $t_{\text {break }}=52$ days. Therefore, the decrease in the X-ray flux at day 50 is also consistent with our wind model. After $t_{\text {break }}$, the hard X-ray becomes weak but lasts about 120 days after the outburst, in other words, until the optically thick wind stopped at $t_{\text {wind }}=120$ days.

6.3. V4743 Sgr $2002 \# 3$
V4743 Sgr (Nova Sagittarii 2002 No.3) was discovered by Haseda on 2002 September 20.431 UT (JD 2452537.931) at mag about 5.0 (Haseda et al. 2002). Kato et al. (2002) reported a prediscovery magnitude of mag 5.5 on September 18.465 UT. This object was not detected by Brown (Brown \& Pearce 2002) on September 9.6 UT (limiting mag about 12.0), so we here assume that $t_{\mathrm{OB}}=$ JD $2452533.0(2002$ September 15.5 UT) is the outburst day.

Bright supersoft X-ray phases were reported at day $\sim 180$ (2003 March 19.396 UT) by Starrfield et al. (2003) (see also Ness et al. 2003) and at day 200 (2003 April 4.921 UT) by Orio et al. (2003). Orio \& Tepedelenlioglu (2004) reported that the supersoft X-ray flux has already declined on 2004 September 30.783 UT (day $\sim 750$ ) by a factor of 1000 . Figure 18 shows the optical and supersoft X-ray light curves. The supersoft X-ray phase started at least $\sim 180$ days after the outburst and ended at day $\sim 400$.

The model of $1.15 M_{\odot}$ WD is a best one among three 1.2 , 1.15, and 1.1 $M_{\odot}$ WDs, as shown in Figure 18. Here we assume a chemical composition of typical neon novae, "Ne nova 2." The $1.15 M_{\odot}$ WD model has $t_{\text {break }}=70, t_{\text {wind }}=180$, and $t_{\mathrm{H} \text {-burning }}=380$ days. Adopting a magnitude of 5.0 on day 3.6 along our model light curve of $1.15 M_{\odot}$ WD, we obtain $t_{2}=9.4$ days and $t_{3}=17$ days as listed in Table 6 . We have estimated the ejecta mass of $M_{\text {wind }} \sim 7 \times 10^{-6} M_{\odot}$. The visual $(V)$ magnitudes are roughly fitted with our model light curve until about day 40, but are gradually departing from it after that. At this stage, the nova probably entered the transition/nebular phase and the deviation comes from the contribution of strong emission lines.

We have also checked the accuracy of WD mass determination that comes from the ambiguity of chemical composition. We obtain a best-reproducing model of $M_{\mathrm{WD}}=1.03 M_{\odot}$ for $X=0.35$ ("Ne nova 1 "), and $M_{\mathrm{WD}}=1.21 M_{\odot}$ for $X=0.65$ ("Ne nova 3"). Then, we have an approximate relation of

$$
M_{\mathrm{WD}}(X) \approx\{1.15+0.6(X-0.55)\} M_{\odot},
$$

for V4743 Sgr. This relation of the WD mass is steeper (on $X)$ than that for V598 Pup and V382 Vel. We may also conclude that the WD mass is $M_{\mathrm{WD}}=1.15 \pm 0.06 M_{\odot}$ for a typical hydrogen content between $X=0.45-0.65$ of V4743 Sgr.

From the FF method, we obtain the distance modulus to V4743 Sgr, i.e.,

$$
\left[(m-M)_{V}\right]_{\mathrm{FF}}=m_{\mathrm{w}}-M_{\mathrm{w}}=14.7-0.9=13.8,
$$

for the $1.15 M_{\odot}$ WD. Two other attendant models of $1.2 M_{\odot}$ and 1.1 $M_{\odot}$ WDs in Figure 18 give similar results of $(m-$ $M)_{V}=14.3-0.7=13.6$ for $1.2 M_{\odot}$ or $(m-M)_{V}=15.2-1.2=$ 14.0 for $1.1 M_{\odot}$ WD.

The LC method gives

$$
\left[5 \log (d / 10)+A_{V}\right]_{\mathrm{V} 1500 \mathrm{Cyg}}-\left[5 \log (d / 10)+A_{V}\right]_{\mathrm{V} 4743 \mathrm{Sgr}}=-1.1 .
$$

With $(m-M)_{V}=12.5$ from Equation (20) for V1500 Cyg, we obtain

$$
\left[(m-M)_{V}\right]_{\mathrm{LC}}=\left[5 \log (d / 10)+A_{V}\right]_{\mathrm{V} 4743 \mathrm{Sgr}}=13.6 .
$$

Kaler-Schmidt's law with $t_{3}=17$ days and Della Valle \& Livio's law with $t_{2}=9.4$ days and $m_{V \text {, max }}=5.0$ yield

$$
\left[(m-M)_{V, \max }\right]_{\mathrm{MMRD}}=\left[5 \log (d / 10)+A_{V}\right]_{\mathrm{MMRD}}=13.7(13.7),
$$




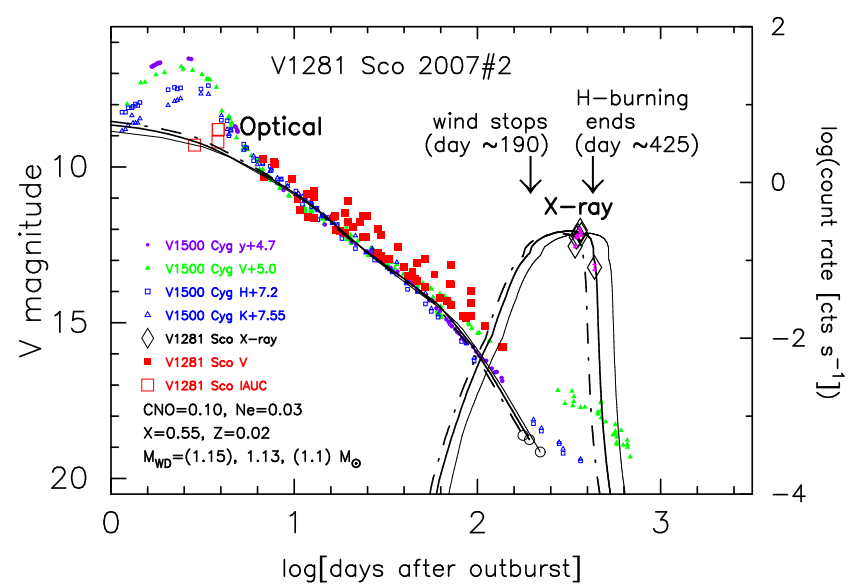

FIG. 19.- Same as Figure 16 but for V1281 Sco. Observational data of optical $V$ (red filled squares) are taken from AAVSO and Association Francaise des Observateurs d'Etoiles Variables (AFOEV). The X-ray count rate data (large open diamonds) with error bars (magenta bars) of V1281 Sco are taken from an automatic analyzer of the Swift web page (Evans et al. 2009) We plot three model light curves of $M_{\mathrm{WD}}=1.15$ (thick dash-dotted lines), 1.13 (thick solid lines), and 1.1 $M_{\odot}$ (thin solid lines) WDs for the envelope chemical composition of "Ne nova 2. ." We select the $1.13 M_{\odot}$ WD as a best model among three of $1.15,1.13$, and $1.1 M_{\odot}$ WDs and can reproduce the supersoft X-ray data.

being consistent with the FF and LC results. Then, the distance modulus to V4743 $\mathrm{Sgr}$ is $(m-M)_{V}=13.7 \pm 0.1$ and the distance is $d=3.8 \pm 0.2 \mathrm{kpc}$ for $A_{V}=3.1 E(B-V)=$ $3.1 \times 0.25=0.78$ (Vanlandingham et al. 2007). Our estimate is also consistent with the recent results of $E(B-V)=0.25$ and $d=3.9 \pm 0.3 \mathrm{kpc}$ by Vanlandingham et al. (2007).

The orbital period of $P_{\text {orb }}=0.2799$ days $(6.72 \mathrm{hr})$ was derived by Kang et al. (2006) from the orbital modulations with a full amplitude of $0.05 \mathrm{mag}$ (2003) - $0.15 \mathrm{mag}$ (2005). Then, we estimate the epoch when the companion emerges from the nova envelope. From Equation (49), we have $M_{2}=0.70 M_{\odot}$, which corresponds to $a=2.2 R_{\odot}$ and $R_{1}^{*}=0.93 R_{\odot}$ for $M_{1}=$ $1.15 M_{\odot}$. It is $t_{\text {emerge }} \approx 36$ days at $R_{\mathrm{ph}} \sim a$.

\subsection{V1281 Sco 2007\#2}

V1281 Sco (Nova Scorpii 2007 No.2) was discovered by Nakamura on 2007 February 19.86 UT (JD 2454151.3593) at mag about 9.3 (Yamaoka et al. 2007). This object was not detected by Nakamura (Yamaoka et al. 2007) on February 14.86 UT (limiting mag about 12.0) and also by $\mathrm{Fu}-$ jita (Naito et al. 2007) on February 18.85 UT (limiting mag about 11.6), so we here assume that $t_{\mathrm{OB}}=\mathrm{JD} 2454148.5$ (2007 February 17.0 UT) is the outburst day.

A bright supersoft X-ray phase was reported at day $\sim$ 340 (2008 January 24.18 UT) by Ness et al. (2008a). To search for the end epoch of supersoft X-ray phase, i.e., $t_{\mathrm{H}-\text { burning }}$, we use an automatic analyzer in the $S$ wift web page ${ }^{1}$ (Evans et al. 2009). The X-ray (0.3-10 keV) count rates with the Swift XRT are plotted in Figure 19 together with its optical light curve. We have checked the early data points of $\mathrm{X}$-ray obtained by automatic analyzer are very similar to the values reported by Ness et al. (2008a). The epoch of $t_{\mathrm{H}-\text { burning }}$ is about 425 days after the outburst, if we regard that the last observation indicates the decay of supersoft X-ray flux.

Our best-reproducing light curve of a $1.13 M_{\odot}$ WD is plotted in Figure 19 for a chemical composition of a typical neon nova, "Ne nova 2 ", which shows $t_{\text {break }}=78, t_{\text {wind }}=190$, and

\footnotetext{
${ }^{1}$ http://www.swift.ac.uk/
}

$t_{\mathrm{H}-\text { burning }}=425$ days after the outburst. We determined the WD mass to be $1.13 \pm 0.03 M_{\odot}$ mainly from the supersoft X-ray data. Adopting a magnitude of 9.2 on day 3.0 along our model light curve of $1.13 \mathrm{M}_{\odot} \mathrm{WD}$, we obtain $t_{2}=9.5$ and $t_{3}=16.7$ days as listed in Table 6 . We have estimated the ejecta mass of $M_{\text {wind }} \sim 9 \times 10^{-6} M_{\odot}$. The visual $(V)$ magnitudes are roughly fitted with our model light curve until about day 80, but are gradually departing from it after that. This deviation comes from the contribution of strong emission lines.

We have also examined the accuracy of WD mass determination that comes from the ambiguity of chemical composition. We obtain a best-reproducing model of $M_{\mathrm{WD}}=1.01 M_{\odot}$ for $X=0.35$ ("Ne nova 1"), and $M_{\mathrm{WD}}=1.2 M_{\odot}$ for $X=0.65$ ("Ne nova 3"). Then, we have

$$
M_{\mathrm{WD}}(X) \approx\{1.13+0.6(X-0.55)\} M_{\odot},
$$

for V1281 Sco. We may conclude that the WD mass is $M_{\mathrm{WD}}=$ $1.13 \pm 0.06 M_{\odot}$ for a typical hydrogen content between $X=$ $0.45-0.65$ of V1281 Sco.

From the FF method, we obtain the distance modulus to V1281 Sco, i.e.,

$$
\left[(m-M)_{V}\right]_{\mathrm{FF}}=m_{\mathrm{w}}-M_{\mathrm{W}}=18.8-1.0=17.8,
$$

for the $1.13 M_{\odot}$ WD model. Two other attendant models of $1.15 M_{\odot}$ and $1.1 M_{\odot}$ WDs in Figure 19 give similar results of $(m-M)_{V}=18.6-0.9=17.7$ for $1.15 M_{\odot}$ or $(m-M)_{V}=$ $19.1-1.2=17.9$ for $1.1 M_{\odot} \mathrm{WD}$.

The LC method gives

$$
\left[5 \log (d / 10)+A_{V}\right]_{\mathrm{V} 1500 \mathrm{Cyg}}-\left[5 \log (d / 10)+A_{V}\right]_{\mathrm{V} 1281 \mathrm{Sco}}=-5.0 .
$$

With $(m-M)_{V}=12.5$ from Equation (20) for V1500 Cyg, we obtain

$$
\left[(m-M)_{V}\right]_{\mathrm{LC}}=\left[5 \log (d / 10)+A_{V}\right]_{\mathrm{V} 1281 \mathrm{Sco}}=17.5 .
$$

Kaler-Schmidt's law with $t_{3}=16.7$ days and Della Valle \& Livio's law with $t_{2}=9.5$ days and $m_{V, \text { max }}=9.2$ (at $t_{0}=3.0$ days) give

$$
\left[(m-M)_{V, \max }\right]_{\mathrm{MMRD}}=\left[5 \log (d / 10)+A_{V}\right]_{\mathrm{MMRD}}=17.9(17.9) \text {, }
$$

being consistent with the FF and LC results, where the difference between the LC method and the others is about \pm 0.4 mag but within the ambiguity in this method (see Sections 4.2 and 6.1). Then the distance modulus is $(m-M)_{V}=17.8 \pm 0.2$ and the distance to V1281 Sco is $d=13 \pm 1 \mathrm{kpc}$ for $A_{V}=$ $3.1 E(B-V)=3.1 \times 0.7=2.17$ (Russell et al. 2007a).

\subsection{V597 Pup 2007\#1}

V597 Pup (Nova Puppis 2007 No.1) was discovered by Pereira on 2007 November 14.23 UT (JD 2454418.73) at mag about 7.0 (Pereira et al. 2007). This object was not detected (Pereira et al. 2007) on November 6.23, 7.22, 8.23, 10.23, and 11.22 UT (limiting mag about 8), so we assume that $t_{\mathrm{OB}}=\mathrm{JD} 2454416.0$ (2007 November 11.5 UT) is the outburst day.

A bright supersoft X-ray phase was reported by Ness et al. (2008c). To search for the end epoch of supersoft X-ray phase, i.e., $t_{\mathrm{H}-\text { burning }}$, we use an automatic analyzer in the Swift web page (Evans et al. 2009). The X-ray $(0.3-10 \mathrm{keV})$ count rates with the Swift XRT are plotted in Figures 20 and 21 as well as the optical light curves. The X-ray data points show that the turnoff of supersoft X-ray is $t_{\mathrm{H} \text {-burning }} \sim 430-460$ 


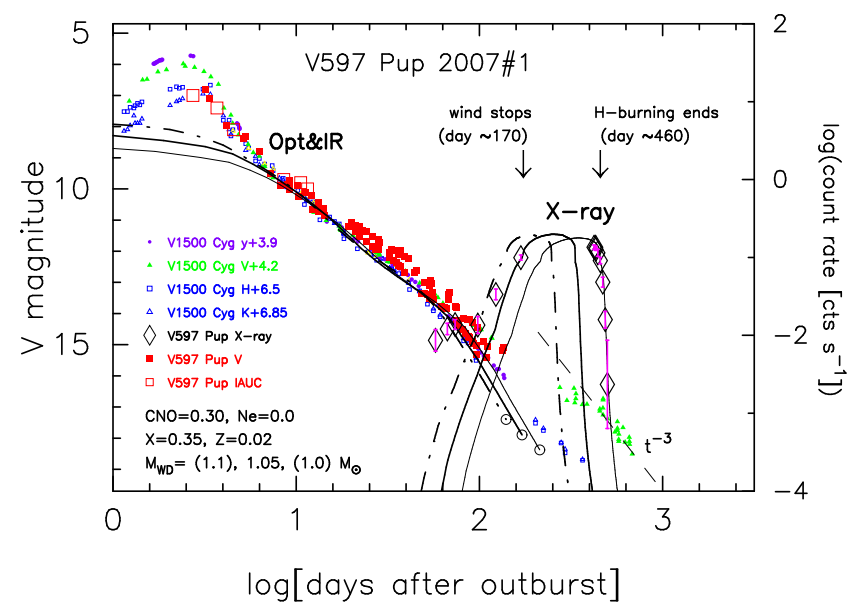

FIG. 20.- Same as Figure 16 but for V597 Pup. We plot three model light curves of $M_{\mathrm{WD}}=1.1$ (thick dash-dotted lines), 1.05 (thick solid lines), and $1.0 M_{\odot}$ (thin solid lines) WDs for the envelope chemical composition of "CO nova 2." We do not select the best model among these three of 1.1, 1.05 , and $1.0 M_{\odot}$ WDs, because the supersoft X-ray duration is too short to be comparable with the observation. The X-ray count rate data $(0.3-10$ $\mathrm{keV}$ : large open diamonds) with error bars (magenta bars) are taken from an automatic analyzer of the Swift web page (Evans et al. 2009). Observational V597 Pup data of $V$ (filled squares) magnitudes are taken from AFOEV and AAVSO.

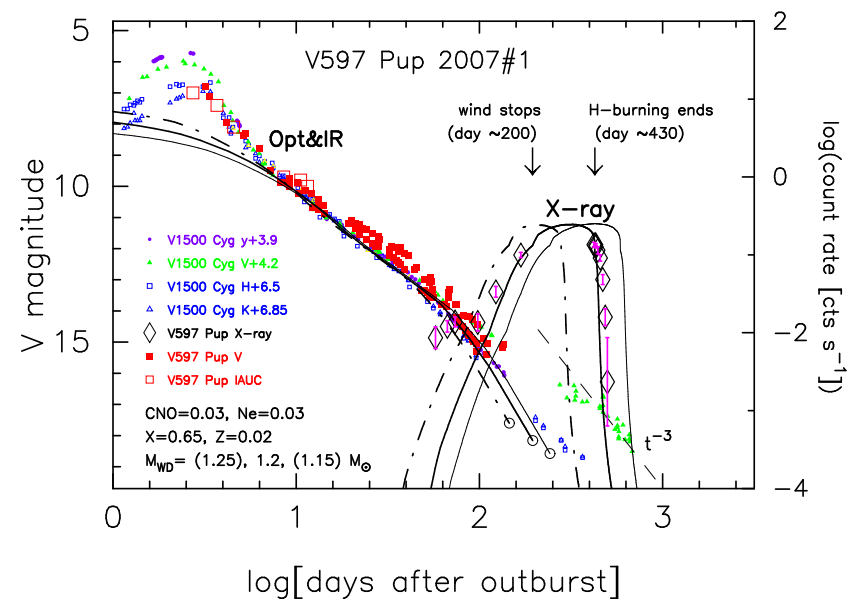

FIG. 21. - Same as Figure 20 but we plot three model light curves of $M_{\mathrm{WD}}=1.25$ (thick dash-dotted lines), 1.2 (thick solid lines), and $1.15 M_{\odot}$ (thin solid lines) WDs for the envelope chemical composition of "Ne nova 3." We select the $1.2 M_{\odot}$ WD as a best model among three of $1.25,1.2$, and $1.15 M_{\odot}$ WDs and can reproduce the supersoft X-ray data.

days and the turn-on is probably $t_{\text {wind }} \sim 150-170$ days. This is a rather rare case in which both the turn-on and turnoff times of supersoft X-ray are specified.

The chemical composition of ejecta is not known, so we first assumed a chemical composition of "CO nova 2 " and made model light curves as shown in Figure 20. The durations of supersoft X-ray phases are too short to be comparable with the observation. This is because the duration of supersoft $\mathrm{X}$ ray is shorter for a smaller hydrogen content of $X$.

Our reasonably-reproducing model light curve of a $1.2 \mathrm{M}_{\odot}$ WD is plotted in Figure 21 for a chemical composition of " $\mathrm{Ne}$ nova 3", which shows $t_{\text {break }}=74, t_{\text {wind }}=200$, and $t_{\mathrm{H} \text {-burning }}=$ 430 days after the outburst. We estimated the WD mass to be $1.2 \pm 0.05 M_{\odot}$ mainly from the supersoft X-ray data. Adopting a magnitude of 8.4 on day 2.7 as the maximum magnitude along our model light curve of $1.2 \mathrm{M}_{\odot} \mathrm{WD}$, we obtain $t_{2}=8.7$ and $t_{3}=16.5$ days as listed in Table 6. We have estimated the ejecta mass of $M_{\text {wind }} \sim 1 \times 10^{-5} M_{\odot}$. The optical light curve shape around the peak deviates largely from our model light curves and is similar to that of V1500 Cyg. It is highly likely that V597 Pup is a superbright nova. After the superbright phase, $V$ magnitudes are roughly fitted with our model light curve until day $\sim 100$.

We have also checked the accuracy of WD mass determination from the ambiguity of chemical composition. For chemical compositions of "Ne nova 1" and "Ne nova 2," however, we could not obtain reasonable fits like those as shown in Figure 21 because supersoft X-ray durations of these models are too short to fit with the supersoft X-ray observation like in Figure 20. Therefore, we may conclude that the WD mass is $M_{\mathrm{WD}}=1.2 \pm 0.05 M_{\odot}$ and that the composition is relatively hydrogen-rich like $X \sim 0.65$.

From the FF method, we obtain the distance modulus to V597 Pup, i.e.,

$$
\left[(m-M)_{V}\right]_{\mathrm{FF}}=m_{\mathrm{w}}-M_{\mathrm{w}}=17.8-0.9=16.9,
$$

for the $1.15 M_{\odot}$ WD ("Ne nova 2") model. Two other attendant models of $1.2 M_{\odot}$ and 1.1 $M_{\odot}$ WDs (not shown in the figure for "Ne nova 2" models), give similar results of $(m-M)_{V}=17.5-0.7=16.8$ for $1.2 M_{\odot}$ or $(m-M)_{V}=$ $18.3-1.2=17.1$ for $1.1 M_{\odot}$ WD. From Figure 20, we also have $(m-M)_{V}=17.3-0.5=16.8$ for $1.1 M_{\odot},(m-M)_{V}=$ $17.8-0.8=17.0$ for $1.05 M_{\odot}$, or $(m-M)_{V}=18.3-1.1=17.2$ for $1.0 M_{\odot}$ WD with "CO nova 2 ."

The LC method gives

$$
\left[5 \log (d / 10)+A_{V}\right]_{\mathrm{V} 1500 \mathrm{Cyg}}-\left[5 \log (d / 10)+A_{V}\right]_{\mathrm{V} 597 \text { Pup }}=-4.2 \text {. }
$$

With $(m-M)_{V}=12.5$ from Equation (20) for V1500 Cyg, we obtain

$$
\left[(m-M)_{V}\right]_{\mathrm{LC}}=\left[5 \log (d / 10)+A_{V}\right]_{\mathrm{V} 597 \text { Pup }}=16.7 .
$$

Kaler-Schmidt's law with $t_{3}=16.5$ days and Della Valle \& Livio's law with $t_{2}=8.7$ days, and both with $m_{V, \max }=8.4$ (at $t_{0}=2.7$ days) yield

$$
\left[(m-M)_{V, \max }\right]_{\mathrm{MMRD}}=\left[5 \log (d / 10)+A_{V}\right]_{\mathrm{MMRD}}=17.1(17.1),
$$

being consistent with the FF and LC results. Then we have $(m-M)_{V}=17.0 \pm 0.2$ and the distance to V597 Pup, $d=16 \pm$ $2 \mathrm{kpc}$, for $A_{V}=3.1 E(B-V)=3.1 \times 0.3=0.93$ (Ness et al. 2008c).

The orbital period of $P_{\text {orb }}=0.1112$ days $(2.6687 \mathrm{hr})$ was derived by Warner \& Woudt (2009) from the orbital modulations with a full amplitude of $0.2 \mathrm{mag}$ (2008) - $0.6 \mathrm{mag}$ (2009). Then, we estimate the epoch when the companion emerges from the nova envelope. From Equation (49), we have $M_{2}=0.22 M_{\odot}$, which corresponds to $a=1.1 R_{\odot}$ and $R_{1}^{*}=0.58 R_{\odot}$ for $M_{1}=1.2 M_{\odot}$. It is $t_{\text {emerge }} \approx 52$ days at $R_{\mathrm{ph}} \sim a$.

Warner \& Woudt (2009) reported that a repetitive hump with an amplitude of $\sim 0.2 \mathrm{mag}$ is seen but no obvious eclipse features in 2008 ( 125 days after outburst). Their Fourier analysis of light curves provide an orbital period of $2.67 \mathrm{hr}$. After that, their 2009 observation revealed broad eclipses with $\sim 0.6$ mag deep ( $\sim 470-500$ days after outburst). They interpreted that the drop in brightness $(2.5 \mathrm{mag})$ between these two observations in 2008 and 2009 was due largely or entirely to the dispersal and cooling of nova ejecta far from the central binary and the eclipse and their related humps could well 


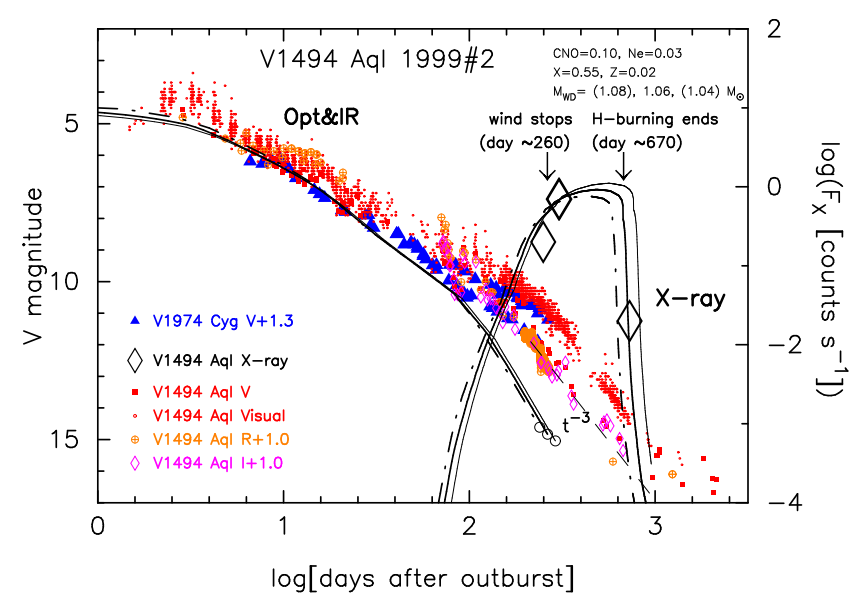

FIG. 22.- Optical and supersoft X-ray light curves of V1494 Aql. Optical bands of visual (small open circles), $V$ (filled squares), $R$ (circles with a plus), and near IR band of I (small open diamonds) magnitudes are shown, which are taken from AAVSO and VSNET. The X-ray count rate data (large open diamonds) without error bars are taken from Rohrbach et al. (2009). We plot three model light curves of $M_{\mathrm{WD}}=1.08$ (thick dash-dotted lines), 1.06 (thick solid lines), and $1.04 M_{\odot}$ (thin solid lines) WDs for the envelope chemical composition of "Ne nova 2 ." We select the $1.06 M_{\odot}$ WD as a best model among three of 1.08, 1.06, and $1.04 M_{\odot}$ WDs and can reproduce the supersoft X-ray data. Observational $V$ magnitudes of V1974 Cyg are added for comparison, which are taken from Chochol et al. (1993).

have been present even in the earlier 2008 light curves, that is, there are some features resembling such diluted structures. This interpretation is consistent with the emergence of companion on $\sim 50\left(R_{\mathrm{ph}}=a\right)$ or $\sim 60$ days $\left(R_{\mathrm{ph}}=a-R_{2}\right)$ after outburst. Moreover, the WD photosphere is larger than that of the companion, i.e., $R_{\mathrm{ph}} \gtrsim R_{2}$, during the optically thick wind phase, at least, until 130 days after outburst. After the winds stop on day $\sim 200$, the WD photosphere rapidly shrinks toward its original size, that is, it becomes a half by one month (see, e.g., Hachisu \& Kato 2003).

\subsection{V1494 Aql 1999\#2}

V1494 Aql (Nova Aquilae 1999 No.2) was discovered by Pereira on 1999 December 1.785 UT (JD 2451514.285) at mag about 6.0 (Pereira et al. 1999). This object was not detected by Liller (Fujii et al. 1999) on November 25.035 UT (limiting mag about 10.5), so we assume that $t_{\mathrm{OB}}=\mathrm{JD}$ 2451513.0 (1999 November 30.5 UT) is the outburst day. The star reached its maximum of $m_{\mathrm{vis}} \approx 3.9$ on 1999 December 3.4 UT (JD 2451515.9) about 3 days after the outburst. Then it declined by 2 mag in 6.6 days and by 3 mag in 16 days (e.g., Kiss \& Thomson 2000). The transition oscillation began on day $\sim 20$ and ended on day $\sim 130$, during which the star exhibited quasi-periodic oscillations with periods of 7 to 22 days and with an amplitude reaching $1.5 \mathrm{mag}$.

We plot the optical and supersoft X-ray light curves in Figure 22. A bright supersoft X-ray phase was detected with Chandra on day 250 (2000 August 6 UT), day 302 (2000 September 28 UT), and day 305 (2000 October 1 UT) (Drake et al. 2003). Ness et al. (2007a) reported that the supersoft X-ray flux had already declined by a factor of 40 on day $\sim 730$ (2001 November 28 UT). Then the supersoft Xray phase started at least on day $\sim 250$ and ended before day $\sim 700$.

Iijima \& Esenoglu (2003) analyzed their spectra of V1494 Aql and estimated the helium/hydrogen ratio to be $\mathrm{He} / \mathrm{H}=$ $0.13 \pm 0.01$ by number ratio, which corresponds to $Y / X=$ $0.52 \pm 0.04$. This helium/hydrogen ratio is close to our chem- ical composition of "Ne nova 2, " in which $Y / X \approx 0.54$. Arkhipova et al. (2002) derived $\mathrm{He} / \mathrm{H}=0.126, \mathrm{~N} / \mathrm{H}=0.04$ (or $0.0015), \mathrm{O} / \mathrm{H}=0.002$, and $\mathrm{Fe} / \mathrm{H}=5 \times 10^{-5}$ by number ratio. This corresponds to $X=0.48, Y=0.24, X_{\mathrm{CNO}}=0.26$, and $Z=0.02$, (or to $X=0.62, Y=0.34, X_{\mathrm{CNO}}=0.02$, and $Z=0.02$ ). We do not know which is better, so that we adopt an arithmetic average of these two, i.e., $X=0.55, Y=0.29, X_{\mathrm{CNO}}=0.14$, and $Z=0.02$, which is close to "Ne nova 2." Therefore, we assume "Ne nova 2"as the envelope chemical composition of V1494 Aql.

Our best-reproducing light curve is a $1.06 M_{\odot}$ WD model among $1.04,1.06$, and $1.08 M_{\odot}$ WDs mainly from the supersoft X-ray data (Figure 22), i.e., $M_{\mathrm{WD}}=1.06 \pm 0.02 M_{\odot}$. For the $1.06 M_{\odot}$ WD model, we derive $t_{\text {break }}=102, t_{\text {wind }}=260$, and $t_{\mathrm{H} \text {-burning }}=670$ days. Adopting a magnitude of $m_{V, \max }=$ 5.0 on day 2.7 as the optical maximum along our model light curve of $1.06 M_{\odot} \mathrm{WD}$, we obtain $t_{2}=11.7$ days and $t_{3}=22.1$ days (Table 6). The visual $(V)$ magnitudes are not well fitted with our model light curve, from day 10 to day 150 , mainly because the light curve has wavy structures and seems to be strongly contaminated by emission lines. Our model light curve almost follows the bottom line of $V$ magnitudes during the transition oscillations, which is supported by Figure 10 for GK Per (see also Figure 2 of Hachisu \& Kato 2007). The nova entered a nebular phase $\sim 100$ days after the outburst and the visual magnitudes deviated much from our model light curve due mainly to strong emission lines such as [O III] (e.g., Iijima \& Esenoglu 2003).

We have also estimated the ejecta mass to be $M_{\text {wind }} \sim 1.1 \times$ $10^{-5} M_{\odot}$ (Table 7), which is lost by the optically thick wind (see, e.g., Kato \& Hachisu 1994; Hachisu \& Kato 2006, for more detail). Iijima \& Esenoglu (2003) derived the ejecta mass of $M_{\text {ej }} \sim(6.2 \pm 1.4) \times 10^{-6} M_{\odot}(d / 1.6 \mathrm{kpc})^{2}$, being consistent with our model estimation if we take the distance of $d=2.2 \mathrm{kpc}$ as derived below.

Based on submillimeter- and centimeter-band fluxes, a single MERLIN image, and optical spectroscopy, Eyres et al. (2005) concluded that optical spectroscopy indicates continued mass ejection for over 195 days, with the material becoming optically thin sometime between 195 and 285 days after the outburst. This is consistent with $t_{\text {wind }}=260$ days of our $1.06 M_{\odot}$ WD model.

Mazuk et al. (2000) reported that V1494 Aql had entered into a strong coronal phase at least 230 days after the outburst. This is closely related to the epoch when the optically thick winds stop, because the photosphere rapidly shrinks and the photospheric temperature quickly increases to $\sim 3 \times 10^{5} \mathrm{~K}$ or more.

We have checked the dependency of the WD mass determination on the hydrogen content of the WD envelope. Kamath et al. (2005) reported a different result of helium/hydrogen ratio of the ejecta from He II $4686 / \mathrm{H} \beta$ ratio, that is, $\mathrm{He} / \mathrm{H}=0.24 \pm 0.06$ by number ratio, which corresponds to $Y / X=0.96 \pm 0.24$ and is close to our "CO nova 2" (see Table 1). When we adopt a chemical composition of "CO nova 2," we obtain a best-reproducing model of $0.92 M_{\odot}$ WD. The other physical parameters are also listed in Tables 6 and 7 On the other hand, if we assume a chemical composition of "Ne nova 3," we obtain a best model of $1.13 M_{\odot}$ WD. Finally we have

$$
M_{\mathrm{WD}}(X) \approx\{1.06+0.7(X-0.55)\} M_{\odot},
$$

for V1494 Aql. Here, we use $M_{\mathrm{WD}}(X=0.65)=1.13 M_{\odot}$, 
$M_{\mathrm{WD}}(X=0.55)=1.06 M_{\odot}$, and $M_{\mathrm{WD}}(X=0.35)=0.92 M_{\odot}$. Then, we may conclude that the WD mass is $M_{\mathrm{WD}}=1.06 \pm$ $0.07 M_{\odot}$ for a typical hydrogen content between $X=0.45-$ 0.65 . If the hydrogen content is $X \sim 0.55$, then the WD has a mass of $1.06 \pm 0.03 M_{\odot}$ and is probably a ONeMg WD. On the other hand, when the hydrogen content is as low as $X \sim 0.35$, then the WD is less massive $\left(0.92 \pm 0.03 M_{\odot}\right)$ and probably a CO WD.

From the FF method, we obtain the distance modulus to V1494 Aql, i.e.,

$$
\left[(m-M)_{V}\right]_{\mathrm{FF}}=m_{\mathrm{w}}-M_{\mathrm{w}}=14.9-1.5=13.4,
$$

for the $1.06 M_{\odot}$ WD ("Ne nova 2") model. Two other attendant models of $1.08 M_{\odot}$ and $1.04 M_{\odot}$ WDs give similar results of $(m-M)_{V}=14.6-1.1=13.5$ for $1.08 M_{\odot}$ or $(m-M)_{V}=15.0-1.7=13.3$ for $1.04 M_{\odot} \mathrm{WD}$.

The LC method gives

$$
\left[5 \log (d / 10)+A_{V}\right]_{\mathrm{V} 1974 \mathrm{Cyg}}-\left[5 \log (d / 10)+A_{V}\right]_{\mathrm{V} 1494 \mathrm{Aql}}=-1.3 .
$$

With $(m-V)_{V}=12.3$ from Equation (16) for V1974 Cyg, we obtain

$$
\left[(m-M)_{V}\right]_{\mathrm{LC}}=\left[5 \log (d / 10)+A_{V}\right]_{\mathrm{V} 1494 \mathrm{Aql}}=13.6 .
$$

Kaler-Schmidt's law with $t_{3}=22.1$ days and Della Valle \& Livio's law with $t_{2}=11.7$ days and $m_{V, \max }=5.0$ (at $t_{0}=2.7$ days) yield

$$
\left[(m-M)_{V, \max }\right]_{\mathrm{MMRD}}=\left[5 \log (d / 10)+A_{V}\right]_{\mathrm{MMRD}}=13.4(13.6),
$$

being consistent with the $\mathrm{FF}$ and $\mathrm{LC}$ results. Then, we obtain the distance modulus of $(m-M)_{V}=13.5 \pm 0.1$ and the distance of $d=2.2 \pm 0.2 \mathrm{kpc}$ to V1494 Aql for $A_{V}=3.1 E(B-V)=$ $3.1 \times 0.6=1.83$ (Iijima \& Esenoglu 2003). This value is a bit larger than the distance of $1.6 \pm 0.2 \mathrm{kpc}$ estimated by Iijima \& Esenoglu (2003) from the MMRD relation.

The orbital period of $P_{\text {orb }}=0.1346$ days $(3.23 \mathrm{hr})$ was derived by Barsukova \& Goranskii (2003) from the orbital modulations with an eclipse depth of $\sim 0.5 \mathrm{mag}$ (see also Retter et al. 2000; Bos et al. 2001). Then, we estimate the epoch when the companion emerges from the nova envelope. From Equation (49), we have $M_{2}=0.28 M_{\odot}$, which corresponds to $a=1.22 R_{\odot}$ and $R_{1}^{*}=0.60 R_{\odot}$ for $M_{1}=1.06 M_{\odot}$. These give $t_{\text {emerge }} \approx 72$ days at $R_{\mathrm{ph}} \sim a$. Various physical parameters are summarized in Tables 6 and 7

Iijima \& Esenoglu (2003) and Kamath et al. (2005) reported that higher ionization lines became prominent about 65 days after the optical maximum (i.e., day $\sim 70$ after the outburst). At that time, the photospheric temperature of our $1.06 M_{\odot}$ WD model has already increased to $T_{\mathrm{ph}} \sim 60000-$ $70000 \mathrm{~K}$ and the higher ionization lines mentioned above are possibly related to this high photospheric temperatures. Another possibility is high temperature optically thin plasma that emits hard X-ray mentioned below, because high temperature plasma that emits hard X-ray emerges around $t_{\text {emerge }} \sim 70$ days.

Hard X-ray was detected with Chandra long before the supersoft X-ray appeared. Rohrbach et al. (2009) reported their six Chandra X-ray spectra. The first three observations were taken with ACIS-I on 134, 187, and 248 days after outburst. The count rates are 1.0, 0.69 and 0.53 counts $^{-1}$, respectively. (We do not plot these point in Figure 22 because the detector ACIS-I is different from that of supersoft X-ray observation,

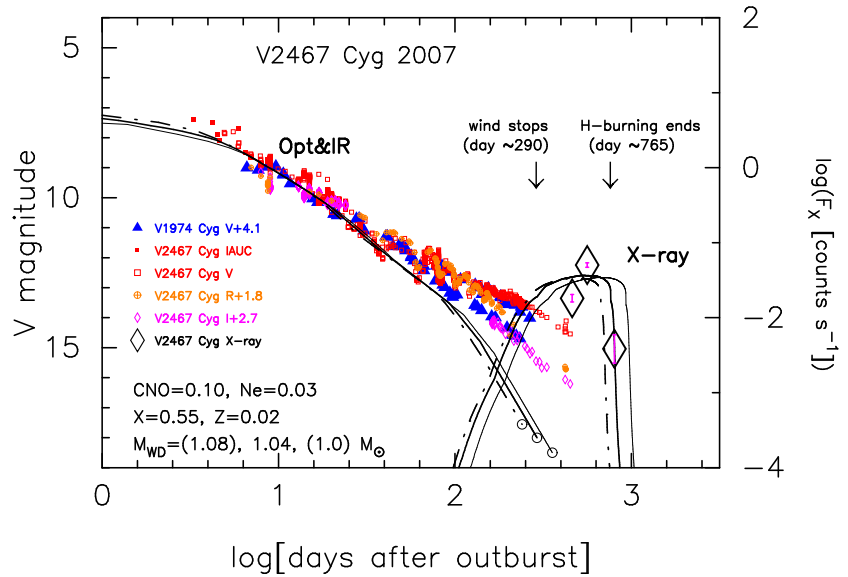

FIG. 23.- Same as Figure 22 but for V2467 Cyg, i.e., optical and supersoft X-ray light curves of V2467 Cyg. Optical V (open red squares), visual (small red filled squares), $R$ (ocher open circles with plus), and $I$ (magenta open diamonds) are taken from IAU Circular 8821, Tomov et al. (2007), and AAVSO, while the supersoft X-ray data (large black diamonds) with error bars (magenta bars) are compiled from Ness et al. (2008b) and also taken from an automatic analyzer of the Swift web page (Evans et al. 2009). We plot three model light curves of $M_{\mathrm{WD}}=1.08$ (thick dash-dotted lines), 1.04 (thick solid lines), and $1.0 \mathrm{M}_{\odot}$ (thin solid lines) WDs for the envelope chemical composition of "Ne nova 2 ." We select the $1.04 M_{\odot}$ WD as a best model among three of $1.08,1.04$, and $1.0 M_{\odot}$ WDs and can reproduce the supersoft $\mathrm{X}$-ray data.

HRC-S.) They found no significant variations in any of these observations. The spectra are dominated by emission lines originating from $\mathrm{N}, \mathrm{O}$, and $\mathrm{Ne}$. They fit isothermal APEC models with the spectra and found best fits with $k T=0.65 \mathrm{keV}$ and $N_{\mathrm{H}}=3.3 \times 10^{21} \mathrm{~cm}^{2}\left(E(B-V)=N_{\mathrm{H}} / 4.8 \times 10^{21}=0.68\right.$ by Ness et al. 2007a). They reported that, in all cases the elemental abundances of $\mathrm{O}$ and $\mathrm{N}$ had to be significantly increased. They further reported that on day 248 a bright soft component appeared in addition to the fading emission lines. When the harder X-ray declined, that is, when the optically thick wind stopped, the soft component of X-ray emerged. This is very consistent with our collision model between the wind and the companion as an origin of high temperature plasma that emits hard X-ray. In our model, the hard component of X-rays emerges around $t_{\text {emerge }} \sim 70$ days and attains its peak at $t_{\text {break }} \sim 100$ days and then declines toward $t_{\text {wind }} \sim 260$ days.

\subsection{V2467 Cyg 2007}

V2467 Cyg (Nova Cygni 2007) was discovered by Tago on 2007 March 15.787 UT (JD 2454175.287) at $7.4 \mathrm{mag}$ (Nakano et al. 2007b). Tago also reported that the nova was fainter than 12th magnitude three days ago (JD 2454172.296). Here we assume the outburst day of $t_{\mathrm{OB}}=$ JD 2454172.0 (2007 March 12.5 UT). In Figure 23, we plot optical and near infrared magnitudes taken from IAU Circular 8821, Tomov et al. (2007), and AAVSO. The nova reached its maximum of $m_{V, \max } \approx 7.4$ at $t_{0} \approx$ JD 2454175.2 (March 16.7 UT). Except for some early, low amplitude oscillations, the light curve declined monotonically until day $\sim 30$. Then V2467 Cyg started its transition phase until day $\sim 150$ in the form of a series of several oscillations with a quasi-period of 20-22 days and a 0.5-1.1 mag amplitude.

Ness et al. (2008b) obtained two Swift observations. The first observation was taken on 2008 June 15.17, about 460 days after the outburst, and obtained an X-ray count rate of 0.017 counts $\mathrm{s}^{-1}$. About 100 days later (2008 September 23.3 
$\mathrm{UT}=566$ days after the outburst), their second observation yields a count rate of 0.048 counts $\mathrm{s}^{-1}$. The both X-ray spectra are soft with a blackbody temperature of $k T=34 \mathrm{eV}$ and $k T=46 \mathrm{eV}$, respectively. These two count rates are plotted in Figure 23. To search for the end epoch of supersoft X-

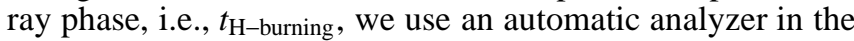
Swift web page (Evans et al. 2009). The three X-ray (0.3-10 $\mathrm{keV})$ data points with the Swift XRT are plotted in Figure 23. early two of which are the same data as those by Ness et al. (2008b). We have checked that the early two data points of the automatic analyzer are very similar to those obtained by Ness et al. (2008b). The epoch of $t_{\mathrm{H} \text {-burning }}$ is around 760 days after the outburst.

Our free-free model light curves are plotted in Figure 23 for a chemical composition of "Ne nova 2." The bottom line of $R$ and $I$ magnitudes are well fitted with our model light curve until about day $\sim 100$. On the other hand, $V$ magnitude is finally departing from the $R$ and $I$ magnitudes from day $\sim 150$ due to strong emission line contributions. The best-reproducing WD mass is $1.04 M_{\odot}$ among $1.08,1.04$, and $1.0 M_{\odot}$. For the $1.04 M_{\odot}$ WD model, we obtain $t_{\text {break }} \approx 113, t_{\text {wind }} \approx 290$, and $t_{\mathrm{H} \text {-burning }} \approx 765$ days. We also obtain "intrinsic" $t_{2} \approx 12$ and $t_{3} \approx 23$ days along our model light curve. Here we assume that the model light curve reached its maximum (7.9 mag) at $t_{0} \sim 3.3$ days after the outburst (at the first observational point).

Swierczynski et al. (2008) reported the possible orbital period of V2467 Cyg, $P_{\text {orb }}=0.1596$ days $(3.82 \mathrm{hr}$ ), from their unfiltered, $R$, and $I$ band CCD photometry. Similar periods of $3.57 \mathrm{hr}$ and $3.85 \mathrm{hr}$ had been already observed in 2007 , several weeks after maximum, by Tomov et al. (2007). We estimate the epoch when the companion emerges from the nova envelope. With the donor mass of $M_{2}=0.35 M_{\odot}$ estimated from Equation (49), we obtain $a=1.38 R_{\odot}$ and $R_{1}^{*}=0.65 R_{\odot}$ for $M_{1}=1.04 M_{\odot}$, giving $t_{\text {emerge }} \approx 75$ days at $R_{\mathrm{ph}} \sim a$.

We have checked the accuracy of WD mass determination from the ambiguity of chemical composition. We obtain a best-reproducing model of $M_{\mathrm{WD}}=0.90 M_{\odot}$ for $X=0.35$ ("CO nova 2"), and $M_{\mathrm{WD}}=1.11 M_{\odot}$ for $X=0.65$ ("Ne nova 3"). Then, we have

$$
M_{\mathrm{WD}}(X) \approx\{1.04+0.7(X-0.55)\} M_{\odot},
$$

for V2467 Cyg. Thus, we may conclude that the WD mass is $M_{\mathrm{WD}}=1.04 \pm 0.07 M_{\odot}$ for a typical hydrogen content between $X=0.45-0.65$. If the hydrogen content is $X \sim 0.55$, then the WD has a mass of $1.04 \pm 0.03 M_{\odot}$ and is probably a ONeMg WD. On the other hand, when the hydrogen content is as low as $X \sim 0.35$, then the WD is less massive $\left(0.90 \pm 0.03 M_{\odot}\right)$ and probably a CO WD.

From the FF method, we obtain the distance modulus to V2467 Cyg, i.e.,

$$
\left[(m-M)_{V}\right]_{\mathrm{FF}}=m_{\mathrm{w}}-M_{\mathrm{w}}=18.0-1.7=16.3,
$$

for the $1.04 M_{\odot}$ WD ("Ne nova 2") model. Two other attendant models of $1.08 M_{\odot}$ and $1.0 M_{\odot}$ WDs give similar results of $(m-M)_{V}=17.6-1.4=16.2$ for $1.08 M_{\odot}$ or $(m-M)_{V}=18.5-2.0=16.5$ for $1.0 M_{\odot}$ WD.

The LC method gives

$$
\left[5 \log (d / 10)+A_{V}\right]_{\mathrm{V} 1974 \mathrm{Cyg}}-\left[5 \log (d / 10)+A_{V}\right]_{\mathrm{V} 2467 \mathrm{Cyg}}=-4.1 .
$$

With $(m-V)_{V}=12.3$ from Equation (16) for V1974 Cyg, we

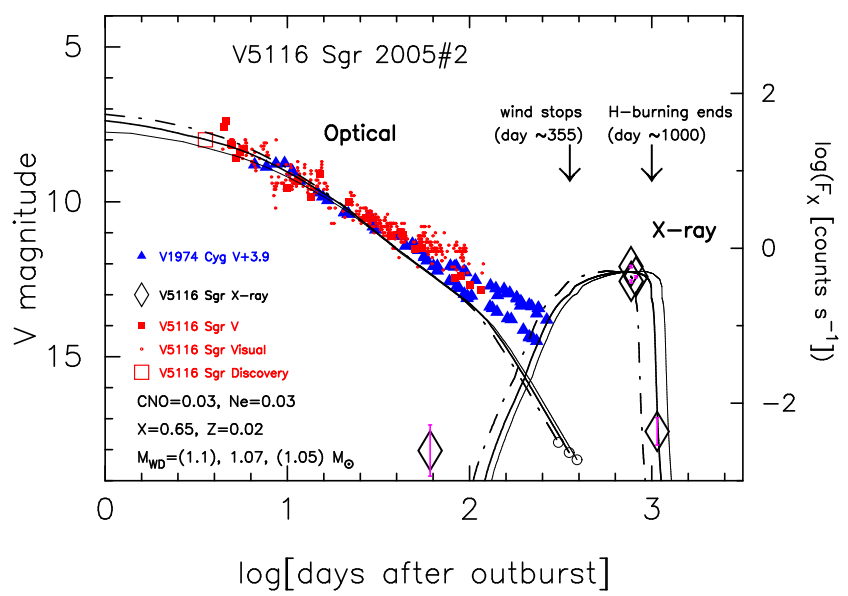

FIG. 24. - Same as Figure 22 but for V5116 Sgr. Optical $V$ (filled red squares), visual (small red open circles), and supersoft X-ray (large diamonds) light curves of V5116 Sgr. The discovery magnitude is denoted by a large open square (Liller 2005). The $V$ and visual data are taken from AAVSO. The X-ray flux data with error bars (magenta bars) are taken from an automatic analyzer of the Swift web page (Evans et al. 2009). We plot three model light curves of $M_{\mathrm{WD}}=1.1$ (thick dash-dotted lines), 1.07 (thick solid lines), and $1.05 M_{\odot}$ (thin solid lines) WDs for the envelope chemical composition of "Ne nova 3." We select the $1.07 M_{\odot}$ WD as a best model among three of $1.1,1.07$, and $1.05 M_{\odot}$ WDs and can reproduce the supersoft $\mathrm{X}$-ray data.

have

$$
\left[(m-M)_{V}\right]_{\mathrm{LC}}=\left[5 \log (d / 10)+A_{V}\right]_{\mathrm{V} 2467 \mathrm{Cyg}}=16.4 .
$$

Kaler-Schmidt's law with $t_{3}=23.0$ days and Della Valle \& Livio's law with $t_{2}=12.0$ days and $m_{V \text {, max }}=7.9$ at maximum (at $t_{0}=3.3$ days) give

$$
\left[(m-M)_{V, \max }\right]_{\mathrm{MMRD}}=\left[5 \log (d / 10)+A_{V}\right]_{\mathrm{MMRD}}=16.2(16.5) \text {, }
$$

being consistent with the FF and LC results. Then, we obtain the distance modulus of $(m-M)_{V}=16.4 \pm 0.2$ and the distance of $d=2.2 \pm 0.2 \mathrm{kpc}$ to V2467 Cyg for $A_{V}=4.65$ (Mazuk et al. 2007).

\subsection{V5116 Sgr 2005\#2}

V5116 Sgr (Nova Sagittarii 2005 No.2) was discovered by Liller on July 4.049 UT (JD 2453555.549) at mag about 8.0 (Liller 2005) and became a supersoft X-ray source (Sala et al. 2008). We have already published analysis of optical light curves for this nova (Hachisu \& Kato 2007) before the supersoft X-ray was detected by Sala et al. (2007). This object was not detected on June 12 (limiting mag about 11.0), so Hachisu \& Kato (2007) assumed that $t_{\mathrm{OB}}=\mathrm{JD} 2453552.0$ (July $1.5 \mathrm{UT}$ ) is the outburst day. Their best-reproducing model is a $0.9 M_{\odot}$ WD for the chemical composition of "CO nova 2." Adopting 8.0 mag on JD 2453555.549 observed by Liller (Liller 2005) as the maximum visual magnitude for V5116 Sgr, Hachisu \& Kato derived $t_{2}=20$ days and $t_{3}=33$ days. From their best light curve of $0.9 M_{\odot}$ WD, Hachisu $\&$ Kato predicted that the supersoft X-ray phase starts on $t_{\text {wind }} \sim 310$ days and ends on $t_{\mathrm{H}-\text { burning }} \sim 770$ days after the outburst.

We plot the supersoft X-ray light curves as well as optical in Figure 24 A bright supersoft X-ray phase was first detected with XMM Newton (Sala et al. 2007) on day 674 (2007 March 5 UT). Sala et al. (2008) reported that the Xray light curve shows abrupt decreases and increases of the flux by a factor of $\sim 8$, which is consistent with a periodic- 
ity of $2.97 \mathrm{hr}$, the orbital period suggested by Dobrotka et al. (2008), and speculated that the X-ray light curve may result from a partial coverage by an asymmetric accretion disk in a high-inclination system. Ness et al. (2007c) also reported the supersoft X-ray detection with Swift on day 768 (2007 August 7.742 UT), and Nelson et al. (2007) observed a similar supersoft X-ray phase on day 789 (2007 August 28 UT). To search for the end epoch of supersoft X-ray phase, i.e.,

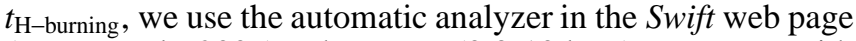
(Evans et al. 2009). The X-ray (0.3-10 keV) count rate with the Swift XRT are plotted in Figure 24. We have checked that the early several data points of the automatic analyzer are very similar to those obtained by Ness et al. (2007c). The epoch of $t_{\mathrm{H} \text {-burning }}$ is somewhere between 820 and 1070 days after the outburst, being roughly consistent with the prediction by Hachisu \& Kato (2007).

Sala et al. (2008) found, from their X-ray spectrum fit, that oxygen/neon-rich WD atmosphere models provide a better fit than carbon/oxygen-rich WD atmosphere models. Therefore, we adopt here the chemical composition of "Ne nova 3 " and recalculated model light curves. Our new best-reproducing model is a $1.07 M_{\odot}$ WD (Figure 24), from which we obtain $t_{\text {break }}=131, t_{\text {wind }}=355, t_{\mathrm{H} \text {-burning }}=1000$ days as listed in Table 6 . The estimated WD mass of $1.07 M_{\odot}$ is very close or nearly equal to the upper limit mass for CO WDs born in a binary, i.e., $M_{\mathrm{CO}} \lesssim 1.07 M_{\odot}$ (e.g., Umeda et al. 1999), being consistent with the suggestion that the WD of V5116 Sgr is an $\mathrm{ONeMg}$ white dwarf.

Adopting a magnitude of $m_{V, \max }=7.9$ at maximum $\left(t_{0}=\right.$ 3.6 days) along our model light curve of the $1.07 M_{\odot} \mathrm{WD}$, we obtain "intrinsic" $t_{2}=15.6$ and $t_{3}=28.5$ days as listed in Table 6. The $V$ magnitudes are roughly fitted with our model light curve at least until day $\sim 100$.

We have checked the accuracy of WD mass determination. We obtain a best-reproducing model of $M_{\mathrm{WD}}=1.0 M_{\odot}$ for $X=0.55$ ("Ne nova 2"), and $M_{\mathrm{WD}}=0.85 M_{\odot}$ for $X=0.35$ ("CO nova 2"). Then, we have

$$
M_{\mathrm{WD}}(X) \approx\{1.07+0.7(X-0.65)\} M_{\odot},
$$

for V5116 Cyg. Only when the hydrogen content is $X \sim 0.65$, the WD has a mass as large as $1.07 M_{\odot}$, which should be consistent with an ONeMg WD. On the other hand, when the hydrogen content is as low as $X \sim 0.35-0.55$, then the WD is less massive $\left(0.85-1.0 M_{\odot}\right)$ and probably a CO WD.

From the FF method, we obtain the distance modulus to V5116 Sgr, i.e.,

$$
\left[(m-M)_{V}\right]_{\mathrm{FF}}=m_{\mathrm{W}}-M_{\mathrm{W}}=18.2-2.0=16.2,
$$

for the $1.0 M_{\odot}$ WD ("Ne nova 2") model. Two other attendant models of $1.05 M_{\odot}$ and $0.95 M_{\odot}$ WDs ("Ne nova 2") give similar results of $(m-M)_{V}=17.6-1.6=16.0$ for $1.05 M_{\odot}$ or $(m-M)_{V}=18.7-2.3=16.4$ for $0.95 M_{\odot} \mathrm{WD}$.

The LC method gives

$$
\left[5 \log (d)+A_{V}\right]_{\mathrm{V} 1974 \mathrm{Cyg}}-\left[5 \log (d)+A_{V}\right]_{\mathrm{V} 5116 \mathrm{Sgr}}=-3.9 \text {. }
$$

With $(m-V)_{V}=12.3$ from Equation (16) for V1974 Cyg, we obtain

$$
\left[(m-M)_{V}\right]_{\mathrm{LC}}=\left[5 \log (d)+A_{V}\right]_{\mathrm{V} 5116 \mathrm{Sgr}}=16.2 .
$$

Kaler-Schmidt's law with $t_{3}=28.5$ days and Della Valle $\&$ Livio's law with $t_{2}=15.6$ days, and both with $m_{V \text {, max }}=7.9$ at

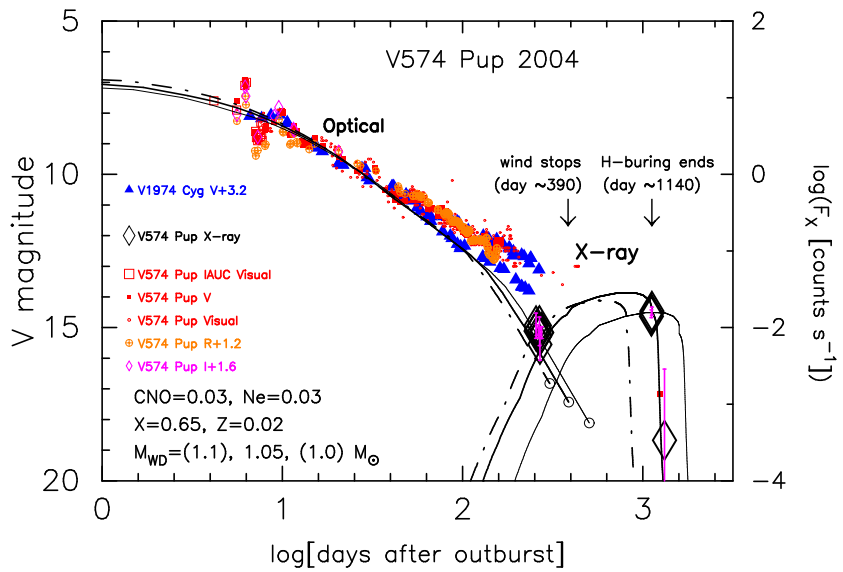

FIG. 25.- Same as Figure 22 but for V574 Pup. We plot three model light curves of $M_{\mathrm{WD}}=1.1$ (thick dash-dotted lines), 1.05 (thick solid lines), and $1.0 M_{\odot}$ (thin solid lines) WDs for the envelope chemical composition of " $\mathrm{Ne}$ nova 3." We select the $1.05 M_{\odot}$ WD as a best model among three of $1.1,1.05$, and $1.0 M_{\odot}$ WDs and can reproduce the supersoft X-ray data. Optical data are taken from AAVSO, Variable Star Observers League in Japan (VSOLJ), and IAU Circulars 8443 and 8445 . The X-ray flux data (large diamonds) with error bars (magenta bars) are taken from Ness et al. (2007a) and from an automatic analyzer of the Swift web page (Evans et al. 2009).

maximum $\left(t_{0}=3.6\right.$ days $)$ yield

$$
\left[(m-M)_{V, \max }\right]_{\mathrm{MMRD}}=\left[5 \log (d)+A_{V}\right]_{\mathrm{MMRD}}=16.0(16.2),
$$

being consistent with the FF and LC results. Then the distance modulus is $(m-M)_{V}=16.2 \pm 0.2$ and the distance to V5116 Sgr is $d=12 \pm 1 \mathrm{kpc}$ for $A_{V}=0.81$ (Burlak 2008). These distance estimates are consistent with $d=11 \pm 3 \mathrm{kpc}$ discussed by Sala et al. (2008).

The orbital period of $P_{\text {orb }}=0.1238$ days $(2.97 \mathrm{hr})$ was derived by Dobrotka et al. (2008) from the orbital modulations with a full amplitude of $0.3-0.4 \mathrm{mag}$. Then, we estimate the epoch when the companion emerges from the nova envelope. From Equation (49), we obtain $M_{2}=0.25 M_{\odot}$, which yields $a=1.15 R_{\odot}$ and $R_{1}^{*}=0.58 R_{\odot}$ for $M_{1}=1.07 M_{\odot}$ and, as a result, $t_{\mathrm{emerge}} \approx 91$ days at $R_{\mathrm{ph}} \sim a$.

\subsection{V574 Pup 2004}

V574 Pup (Nova Puppis 2004) was discovered independently by Tago and Sakurai on 2004 November 20.672 UT (JD 2453330.172) at mag about 7.6 (Nakano et al. 2004). This object was not detected on November 12 and 16 with limiting mag about 13.6 (Nakano et al. 2004), so we assume that $t_{\mathrm{OB}}=\mathrm{JD} 2453326.0$ (November 16.5 UT) is the outburst day. The optical and near infrared light curves are plotted in Figure 25. Although Siviero et al. (2005) derived $m_{V, \max } \approx$ 8.0 on JD 2453335.6 (November 26.1 UT), this is not the $V$ maximum but the second peak as can be seen in Figure 25 We derive $m_{V \text {, } \max } \approx 7.0$ at $t_{0} \approx \mathrm{JD} 2453332.0$ (November 22.5 UT), 6 days after the outburst based on the data reported in IAU Circular 8445 (Sostero et al. 2004). After the $V$ magnitude drops by about $2 \mathrm{mag}$ at $t \approx \mathrm{JD} 2453333.7$ (November $24.2 \mathrm{UT}$ ), it increases to $8.0 \mathrm{mag}$ at $t \approx \mathrm{JD} 2453335.6$ (November 26.1 UT). After that, the $V$ magnitude declines to about 9.0 mag and stays for 20 days and then declines almost monotonically. In this way, V574 Pup shows an oscillatory feature in the very early phase.

Swift XRT observations of V574 Pup showed that the nova became a supersoft X-ray source before 2005 August 17 (Ness et al. 2007a), that is, 274 days after the outburst. 
Ness et al. (2007b) reported that V574 Pup was still in a supersoft X-ray phase on 2007 December 7 and 13, that is, 1121 days after the outburst. To search for the end epoch of supersoft X-ray phase, we use the automatic analyzer in the Swift web page (Evans et al. 2009). The X-ray (0.3-10 keV) count rate with the Swift XRT are plotted in Figure 25. We added the last observational point from Swift web page. The epoch of $t_{\mathrm{H}-\text { burning }}$ is somewhere between 1130 and 1320 days after the outburst.

Rudy et al. (2006) suggested that the observational properties of V574 Pup were intermediate between those typical of explosions on a CO or an ONeMg WD. Their Spitzer observation shows that V574 Pup reveals the strong coronal lines of $\mathrm{Mg}$ and $\mathrm{Ne}$ ions (but no Ne II at 12.8 microns), the behavior of which is suggestive of an explosion on the surface of an $\mathrm{ONeMg} \mathrm{WD}$. Therefore, we have calculated light curves for three different chemical compositions, i.e., "Ne nova 3," "Ne nova 2," and "CO nova 2." However, only the case of "Ne nova 3" provides a reasonable fit to the observation. Our best-reproducing light curve is plotted in Figure 25 for the chemical composition of "Ne nova 3." The WD mass is estimated to be $\sim 1.05 \pm 0.05 M_{\odot}$, which suggests a possibility that the WD is an ONeMg WD, because the estimated WD mass of $1.05 M_{\odot}$ is marginally consistent with the theoretically obtained upper limit mass for CO WDs born in binaries, $M_{\mathrm{CO}} \lesssim 1.07 M_{\odot}$ (e.g., Umeda et al. 1999).

For the other two chemical compositions, i.e., "Ne nova 2," and "CO nova 2," we do not find any reasonable light curves which simultaneously satisfy both the start and end of supersoft X-ray phase, that is, the duration of supersoft X-ray phase is too short to be compatible with the observation, like as has already shown in Figure 20 .

We determine $t_{2}$ and $t_{3}$ along our model light curve. Assuming that our model light curve reached its maximum at the discovery day $\left(t_{0}=4.172\right.$ days after the outburst), we obtain "intrinsic" $t_{2}=18.6$ and $t_{3}=35$ days. The other timescales are obtained to be $t_{\text {break }}=144, t_{\text {wind }}=390, t_{\mathrm{H}-\text { burning }}=1140$ days (Table 6).

From the FF method, we obtain the distance modulus to V574 Pup, i.e.,

$$
\left[(m-M)_{V}\right]_{\mathrm{FF}}=m_{\mathrm{w}}-M_{\mathrm{w}}=17.4-2.0=15.4,
$$

for the $1.0 M_{\odot}$ WD ("Ne nova 2") model, although the models of "Ne nova 2" are not shown in the figure. Two other attendant models of $1.04 M_{\odot}$ and $0.95 M_{\odot}$ WDs ("Ne nova 2") give similar results of $(m-M)_{V}=16.9-1.7=15.2$ for $1.04 M_{\odot}$ or $(m-M)_{V}=17.8-2.3=15.5$ for $0.95 M_{\odot} \mathrm{WD}$.

The LC method gives

$$
\left[5 \log (d / 10)+A_{V}\right]_{\mathrm{V} 1974 \mathrm{Cyg}}-\left[5 \log (d / 10)+A_{V}\right]_{\mathrm{V} 574 \text { Pup }}=-3.2 .
$$

With $(m-V)_{V}=12.3$ from Equation (16) for V1974 Cyg, we have

$$
\left[(m-M)_{V}\right]_{\mathrm{LC}}=\left[5 \log (d / 10)+A_{V}\right]_{\mathrm{V} 574 \text { Pup }}=15.5 .
$$

Kaler-Schmidt's law with $t_{3}=35.0$ days or Della Valle \& Livio's law with $t_{2}=18.6$ days and $m_{V, \max }=7.6$ at maximum $\left(t_{0} \approx 4.2\right.$ days $)$ give

$\left[(m-M)_{V, \max }\right]_{\mathrm{MMRD}}=\left[5 \log (d / 10)+A_{V}\right]_{\mathrm{MMRD}}=15.5(15.7)$,

being consistent with the $\mathrm{FF}$ and $\mathrm{LC}$ results. Thus the distance modulus is $(m-M)_{V}=15.5 \pm 0.2$ and the distance to V574

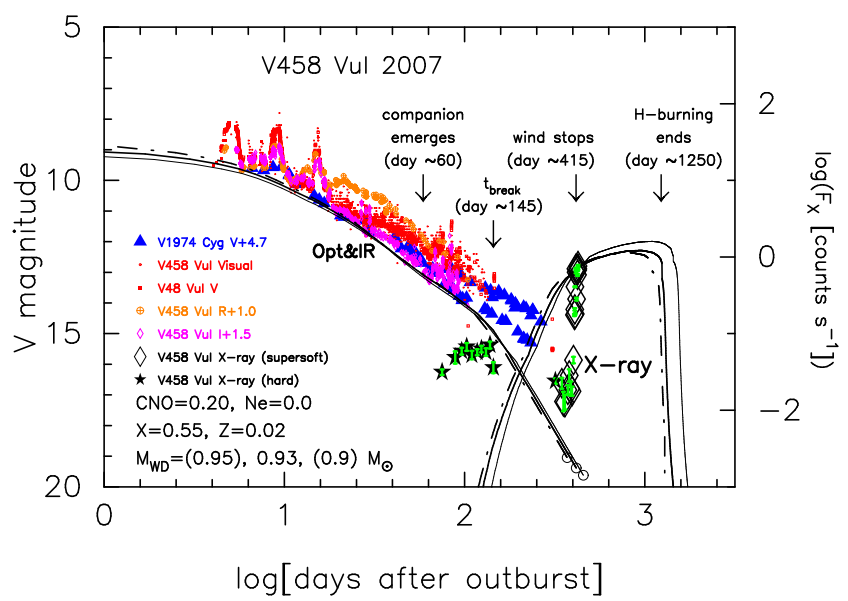

FIG. 26. - Same as Figure 22 but for V458 Vul. Optical $V$ (filled red squares), visual (small red open circles), $R$ (ocher circles with plus), and $I$ (magenta open diamonds) are taken from AAVSO and VSOLJ. The X-ray flux data (filled star marks for hard and open large diamonds for supersoft $\mathrm{X}$-ray) with error bars (green bars) are taken from Ness et al. (2009). We plot three model light curves of $M_{\mathrm{WD}}=0.95$ (thick dash-dotted lines), 0.93 (thick solid lines), and $0.9 M_{\odot}$ (thin solid lines) WDs for the chemical composition of "CO nova 4 ." We obtain $t_{\text {wind }}=370,416$, and 456 days, for the $0.95,0.93$, and $0.9 M_{\odot}$ WDs, respectively. Therefore we select the $0.93 M_{\odot}$ WD as a best model among three of $0.95,0.93$, and $0.9 M_{\odot}$ WDs by fitting our model value of $t_{\text {wind }}$ with the observed turn-on of supersoft X-ray, i.e., $t_{\text {wind }} \approx t_{\mathrm{X} \text {-on }}=415 \pm 15$ days, because supersoft X-ray is self-absorbed by wind itself. We also indicate the epoch when the companion emerges from the photosphere and the break point of optical light curve in order to explain the flux of hard X-ray (filled star marks).

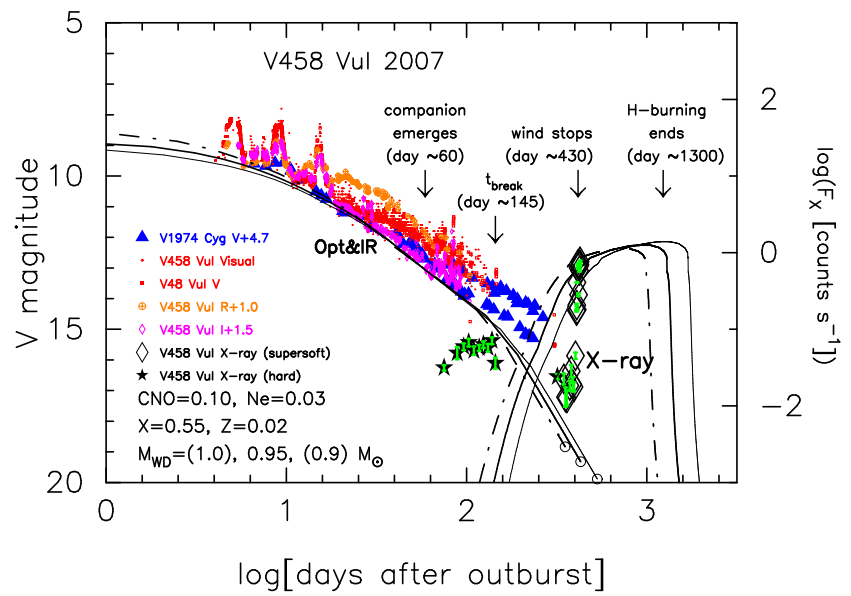

FIG. 27.- Same as Figure 26 but for chemical composition of "Ne nova 2." We plot three model light curves of $M_{\mathrm{WD}}=1.0$ (thick dash-dotted lines), 0.95 (thick solid lines), and $0.9 M_{\odot}$ (thin solid lines) WDs. We obtain $t_{\text {wind }}=$ 352,430 , and 531 days, for the $1.0,0.95$, and $0.9 M_{\odot}$ WDs, respectively. Therefore we select the $0.95 M_{\odot} \mathrm{WD}$ as a best model among three of 1.0 , 0.95 , and $0.9 M_{\odot}$ WDs.

Pup is $d=4.6 \pm 0.3 \mathrm{kpc}$ for $A_{V}=2.2$ (Burlak 2008).

\subsection{V458 Vul 2007}

V458 Vul (Nova Vulpeculae 2007) was discovered by Abe on 2007 August 8.54 UT (JD 2454321.04) at mag about 9.5 (Nakano et al. 2007a). This object was not detected on July 23, 31, and August 4 with limiting mag about 11.5 (Nakano et al. 2007a), so we assume that $t_{\mathrm{OB}}=\mathrm{JD} 2454317.0$ (August 4.5 UT) is the outburst day. The light curve is shown in Figure 26. The $V$ magnitudes show a striking multi-peak (or multi-pulse) structure in the early phase. This kind of multi-peak structures were observed in HR Del 1967 and V723 Cas 1995, both of which are considered to be a nova 
on a CO WD. So we assume here that V458 Vul was also a nova on a $\mathrm{CO}$ WD.

Drake et al. (2007) reported the supersoft X-ray detection of V458 Vul with the Swift XRT on 2007 October 18.05 UT (75 days after the outburst). Tsuiimoto et al. (2008), however, concluded, with the Suzaku observations, that the spectrum can be described by a single temperature $(\sim 0.64 \mathrm{keV})$ thin thermal plasma model, suggesting shock-origin of the Xray. Recently, Drake et al. (2008) reported, with the Swift XRT observations obtained from 2008 June 18 to September 16 (about 320-410 days after the outburst), that V458 Vul have entered a new phase characterized by a highly variable supersoft X-ray component accompanied by partially anti-correlated variations in the ultraviolet. They also pointed that an earlier report on entry into the supersoft phase (Drake et al. 2007) has proven premature. Ness et al. (2009) reported a summary of the early phase X-ray observation with Swift in which a supersoft X-ray phase started about 400 days after the outburst. Figure 26 shows the supersoft X-ray fluxes reported in Ness et al. (2009). The spectra show hard (star marks) components similar to Tsujimoto et al.'s until day $\sim 400$ and then turned into supersoft (open diamonds).

The chemical composition of V458 Vul is not yet reported. So we here assume "CO nova 4" in Table 1, which is similar to that of HR Del (Tylenda 1978) and V723 Cas (Iijima 2006). Our model light curves are plotted in Figure 26. This nova has an oscillation phase in the early $V, R$, and $I$ light curves. We thus fit our universal decline law with the bottom line of each oscillation as shown in the figure. The bottom line of $I$ magnitudes is nicely fitted with our model light curve until day $\sim 100$. On the other hand, the other two, $V$ and $R$ magnitudes, are gradually departing from the model light curve due to emission line contributions.

In the previous subsections, we have determined the WD masses mainly from X-ray light curve fitting because optical and near IR model light curves (i.e., free-free emission model light curves) have a similar shape of light curves and are not sensitive to the WD mass as explained in Figure 8 In the case of V458 Vul, only the emergence of supersoft X-ray was clearly detected but the supersoft X-ray phase does not end yet. Therefore, we have to estimate the WD mass only from the $t_{\mathrm{X}-\text { on }}$ time.

The supersoft X-ray flux replaced the hard X-ray component and rose up around $t \sim 415$ days as shown in Figure 26 As shown in V382 Vel, we proposed a hypothesis that hard $\mathrm{X}$-ray component is a shock-origin by optically thick winds. Therefore, the substantial flux of hard X-ray indicates that optically thick winds still blow during the hard X-ray phase. On the other hand, supersoft X-ray may be self-absorbed by wind itself. Therefore, the emergence of supersoft X-ray indicates that the optically thick wind has stopped or substantially weakened (e.g., Hachisu \& Kato 2003).

Our supersoft X-ray model light curves show a gradual rise in Figure 26 because our flux is the photospheric flux and does not include the effect of absorption by winds. Here we suppose that supersoft X-ray is absorbed during the optically thick wind phase. The sudden rise in the supersoft X-ray flux just after the optically thick winds stopped matches our view of dense optically thick winds (see, e.g., Hachisu \& Kato 2003, for a sharp increase/decrease supersoft X-ray flux of RX J0513.9-6951).

The date of supersoft X-ray emergence is sharply determined to be $t_{\text {wind }}=415 \pm 15$ days for V458 Vul. We have calculated three models of $0.95,0.93$, and $0.9 M_{\odot}$ WDs and obtained $t_{\text {wind }}=370,416$, and 456 days, respectively. Then the WD mass is estimated to be $M_{\mathrm{WD}} \sim 0.93 \pm 0.03 M_{\odot}$ for "CO nova 4" from the emergence time of supersoft Xray, i.e., $t_{\text {wind }} \approx t_{\mathrm{X} \text {-on }} \sim 415$ days. Here we regard that the sharp increase in the supersoft X-ray flux corresponds to the end epoch of optically thick winds at about day 415. For the best model of $M_{\mathrm{WD}}=0.93 M_{\odot}$ among $0.95,0.93$, and $0.9 M_{\odot}$ WDs, we obtain $t_{\text {break }} \approx 145$ days, $t_{\text {wind }} \approx 415$ days, and $t_{\mathrm{H} \text {-burning }} \approx 1250$ days (2011 January 5.5 UT) as listed in Table 6 .

We have checked the accuracy of WD mass determination especially for $\mathrm{CNO}$ abundance. We adopt other chemical composition of "Ne nova 2," same hydrogen content $X=0.55$ but different $\mathrm{CNO}$ abundance $X_{\mathrm{CNO}}=0.1$ vs. 0.2 , and we obtain $t_{\text {wind }}=352,430$, and 531 days for $M_{\mathrm{WD}}=1.0,0.95$, and $0.9 M_{\odot}$ WDs, respectively. Here we adopt the bestreproducing model of $M_{\mathrm{WD}}=0.95 M_{\odot}$ among these three WD masses as shown in Figure 27. This result may suggest that

$$
M_{\mathrm{WD}}\left(X_{\mathrm{CNO}}\right) \approx\left\{0.93-0.2\left(X_{\mathrm{CNO}}-0.2\right)\right\} M_{\odot},
$$

for V458 Vul. The slope of -0.2 for CNO abundance is much smaller than that $(\sim 0.6)$ for hydrogen content. Therefore, if we regard the usual range of chemical composition as $X_{\mathrm{CNO}}=0.1-0.3$ and $X=0.45-0.65$, the ambiguity in WD mass determination $\left( \pm 0.02 M_{\odot}\right)$ for $\mathrm{CNO}$ abundance is much smaller than that $\left( \pm 0.06 M_{\odot}\right)$ for hydrogen content. Therefore it is likely that $M_{\mathrm{WD}}=0.93 \pm 0.08 M_{\odot}$ for a broad range of the chemical composition $X_{\mathrm{CNO}}=0.1-0.3$ $\left(X_{\mathrm{CNO}}=0.2 \pm 0.1\right)$ and $X=0.45-0.65(X=0.55 \pm 0.1)$.

We have estimated $t_{2}$ and $t_{3}$ along our model light curve. Placing our model light curve on the bottom line of multipulse light curves as shown in Figure 26 and assuming that the $V$ magnitude attains its maximum of $\sim 9.4$ mag at $t_{0} \sim 4.4$ days, we obtain "intrinsic" $t_{2}=20.7$ and $t_{3}=38.7$ days. The assumed value of our theoretical peak is $\sim 1.4$ mag dimmer than that of the observed peak $(8.0 \mathrm{mag})$.

From the FF method, we obtain the distance modulus to V458 Vul, i.e.,

$$
\left[(m-M)_{V}\right]_{\mathrm{FF}}=m_{\mathrm{w}}-M_{\mathrm{w}}=19.3-2.3=17.0,
$$

for the $0.95 M_{\odot}$ WD ("Ne nova 2 ") as shown in Figure 27 Two other attendant models of $1.0 M_{\odot}$ and $0.9 M_{\odot}$ WDs give similar results of $(m-M)_{V}=18.8-2.0=16.8$ for $1.0 M_{\odot}$ or $(m-M)_{V}=19.9-2.7=17.2$ for $0.9 M_{\odot}$ WD. Here we do not use the model light curves of "CO nova 4," because they are not calibrated yet.

The LC method gives

$$
\left[5 \log (d / 10)+A_{V}\right]_{\mathrm{V} 1974 \mathrm{Cyg}}-\left[5 \log (d / 10)+A_{V}\right]_{\mathrm{V} 458 \mathrm{Vul}}=-4.7 .
$$

With $(m-V)_{V}=12.3$ from Equation (16) for V1974 Cyg, we have

$$
\left[(m-M)_{V}\right]_{\mathrm{LC}}=\left[5 \log (d / 10)+A_{V}\right]_{\mathrm{V} 458 \mathrm{Vul}}=17.0 .
$$

Kaler-Schmidt's law with $t_{3}=37.2$ days or Della Valle \& Livio's law with $t_{2}=20.4$ days, and both with $m_{V \text {, } \max }=9.3$ at maximum $\left(t_{0}=4.4\right.$ days $)$ yield

$$
\left[(m-M)_{V, \max }\right]_{\mathrm{MMRD}}=\left[5 \log (d / 10)+A_{V}\right]_{\mathrm{MMRD}}=17.1(17.3) \text {, }
$$

being consistent with the FF and LC results. Thus we obtain the distance modulus of $(m-M)_{V}=17.1 \pm 0.2$ and the distance of $d=11 \pm 1 \mathrm{kpc}$ to V458 Vul for $A_{V}=3.1 E(B-V)=3.1 \times$ 
$0.6=1.86$ (Lynch et al. 2008; Poggiani 2008; Wesson et al. 2008). This distance is roughly consistent with $d=13 \pm 3 \mathrm{kpc}$ estimated by Wesson et al. (2008).

Finally, we introduce some characteristic features concerning binary nature, that is, the emergence of the companion from the WD photosphere. Goranskij et al. (2008) reported the orbital period of $P_{\text {orb }}=0.58946$ day with a full amplitude of 0.4 mag. The orbital light curve shows a saw-tooth shape similar to those in other supersoft X-ray novae, e.g. CI Aql and V723 Cas. Because Equation (49) is not applicable to such a long orbital period of $P_{\text {orb }}=14.15 \mathrm{hr}$, we adopt a condition that the companion mass is smaller than $0.74 M_{\odot}$. This condition comes from $q=M_{2} / M_{1} \lesssim 0.8$ $\left(M_{1}=M_{\mathrm{WD}}=0.93 M_{\odot}\right)$ required for thermally stable mass transfer in a binary (e.g., Webbink et al. 1983). If we assume $M_{2}=0.74 M_{\odot}$ as an upper limit of the secondary mass, then we obtain $a=3.5 R_{\odot}$ and $R_{1}^{*}=1.4 R_{\odot}$. It is $t_{\text {emerge }} \approx 62$ days at $R_{\mathrm{ph}} \sim a$. Even if we take a smaller mass of $M_{2}=0.5 M_{\odot}$, we obtain similar values of $a=3.3 R_{\odot}, R_{1}^{*}=1.45 R_{\odot}$, giving $t_{\text {emerge }} \approx 65$ days at $R_{\mathrm{ph}} \sim a$.

Tsujimoto et al. (2008) explained the early Swift X-ray data as shock-origin, which started about 70 days after the outburst and peaked at about 140 days and then decreased as can be seen in Figure 26. The shock may be formed by collision between two ejecta shells (e.g., Friedjung 1987; Cassatella et al. 2004) or by collision between nova winds and the companion star (Hachisu \& Kato 2005, 2006; Hachisu et al. 2008).

If these X-rays come from the shock between the optically thick WD wind and the companion, the emergence of hard Xrays should be coincident with the emerge of the companion from the WD photosphere. This is roughly consistent with $t_{\text {emerge }} \approx 65$ days. On the other hand, the break of free-free emission light curve is caused by a more steeper decrease in the wind mass-loss rate after the break point. Therefore, the drop of X-ray flux at/near 140 days after the outburst is also consistent with our model of $t_{\text {break }} \approx 145$ days. The hard Xray lasted at least until about $t_{\text {wind }} \approx 410$ days, that is, until when the optically thick wind stopped.

It should be noted that, for the orbital period of V458 Vul, $\mathrm{R}$. Wesson et al. (private communication) proposed another orbital period of $98.1 \mathrm{~min}$. If it is the case, the orbital period of $P_{\text {orb }}=0.0681$ day $(1.635 \mathrm{hr})$ is much shorter than that obtained by Goranskij et al. (2008) and this period gives $M_{2}=0.12 M_{\odot}$ from Equation (49), $a=0.71 R_{\odot}, R_{1}^{*}=0.40 R_{\odot}$, and $t_{\text {emerge }} \approx 136$ days (at $R_{\mathrm{ph}} \sim a$ ). Then, the emergence time of the companion star is too late, that is, $t_{\text {emerge }} \sim t_{\text {break }} \sim 140$ days, and our model on the origin of hard X-ray may not be viable.

\subsection{Results on prediction formulae and t time}

In the previous subsections, we have fitted our model light curves with those of ten classical novae, in which a supersoft $\mathrm{X}$-ray phase was detected, and estimated various nova parameters. Figure 28] shows a summary of our results, that is, $t_{\mathrm{X}-\mathrm{off}}$, $t_{\mathrm{X}-\mathrm{on}}$, and $t_{2}$ (from top to bottom) against $t_{3}$ for the ten novae, excluding V598 Pup but instead including V1974 Cyg, which was studied by Hachisu \& Kato (2006).

This figure shows a very good agreement between the data of "intrinsic" $\left(t_{2}, t_{3}\right)$ and Equation (29). This is not surprising because Equation (29) can be derived from the universal decline law with $F_{\lambda} \propto t^{-1.75}$ as shown in Figures 3 and 6 (see also Hachisu \& Kato 2006), and both $t_{2}$ and $t_{3}$ are calculated from our model light curve of the universal decline law.

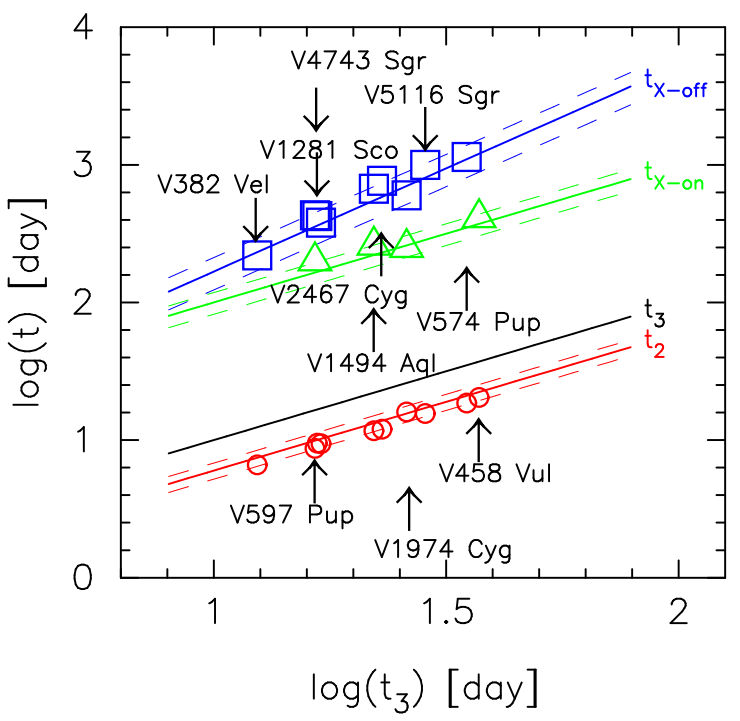

FIG. 28.- Supersoft X-ray on/off and "intrinsic" $t_{2}$ time are plotted against "intrinsic" $t_{3}$ time for 10 novae, V1974 Cyg 1992, V382 Vel 1999, V1494 Aql 1999\#2, V4743 Sgr 2002, V574 Pup 2004, V5116 Sgr 2005, V1281 Sco 2007, V2467 Cyg 2007, V458 Vul 2007, and V597 Pup 2007\#1. From top to bottom, $t_{\mathrm{X}-\mathrm{off}}, t_{\mathrm{X}-\mathrm{on}}$, and intrinsic $t_{2}$ in units of day. Solid lines: central values of Equations (31), (30), and (29). Upper/lower dashed lines: upper/lower values of Equations 31, 30, and 29), respectively from top to bottom. Each value of $t_{\mathrm{X} \text {-off }} \approx t_{\mathrm{H} \text {-burning }}$ (open squares), $t_{\mathrm{X} \text {-on }} \approx t_{\text {wind }}$ (open triangles), intrinsic $t_{2}$ (open circles), and $t_{3}$ is taken from Table 6

Figure 28 also shows reasonable agreement of the observed $t_{\mathrm{X}-\mathrm{on}}$ and $t_{\mathrm{X}-\mathrm{off}}$ against $t_{3}$ with Equations (30) and (31), respectively. Theoretically, $t_{\mathrm{X}-\mathrm{on}}(\approx$ the epoch when optically thick winds stop) and $t_{\mathrm{X} \text {-off }}(\approx$ the epoch when hydrogen-shell burning stops) are uniquely determined if the WD mass and the chemical composition are given. However, $t_{2}$ and $t_{3}$ times are theoretically not unique even if both the WD mass and the chemical composition are given. These timescales depend further on the initial envelope mass as already explained in Section 3 . Therefore, we must obtain $t_{2}$ and $t_{3}$ times from each outburst of various novae. Good agreement between our prediction formulae and the observation on $t_{\mathrm{X}-\mathrm{on}}$ and $t_{\mathrm{X}-\mathrm{off}}$ suggests that our nova models of optically thick winds and freefree model light curves are reasonable for the entire description of nova evolutions and, at the same time, the scatter in the initial nova envelope mass is not so large from their average. In our modeling, we estimated their average relations, $t_{3}$ vs. $t_{\text {wind }}$ or $t_{3}$ vs. $t_{\mathrm{H}-\text { burning }}$, from the model of V1668 Cyg. Such a relatively small scatter of the 10 novae from these relations strongly suggests that we reasonably predict the supersoft Xray phases of novae from this diagram, i.e., from Equations (30) and (31) if we can estimate accurate "intrinsic" $t_{2}$ or $t_{3}$ time from early optical light curves.

\section{CONCLUSIONS}

Our main conclusions consist of three parts: the first part is for basic properties of our model light curves which may be summarized as follows:

1. We model optical and infrared light curves with freefree emission, and UV $1455 \AA$ and supersoft X-ray light curves with blackbody emission, based on the optically thick wind model, as done in our previous paper (Hachisu \& Kato 2006). The model parameters governing the light curves are the WD mass $\left(M_{\mathrm{WD}}\right)$, chemical composition of the WD envelope $\left(X, Y, Z, X_{\mathrm{CNO}}\right.$, and $\left.X_{\mathrm{Ne}}\right)$, and the initial envelope mass $\left(M_{\mathrm{env}, 0}\right)$. 
TABLE 8

Distance Moduli of Novae

\begin{tabular}{|c|c|c|c|c|c|c|c|c|c|c|}
\hline object & $\ldots$ & $\begin{array}{c}t_{2} \\
\text { (day) }\end{array}$ & $\begin{array}{c}t_{3} \\
\text { (day) }\end{array}$ & $m_{V, \text { max }}$ & $\begin{array}{c}(m-M)_{V} \\
\mathrm{FF}^{\mathrm{a}}\end{array}$ & $\begin{array}{c}(m-M)_{V} \\
\text { LC }^{\mathrm{b}}\end{array}$ & $\begin{array}{l}(m-M)_{V} \\
\text { MMRD }^{\mathrm{c}}\end{array}$ & $A_{V}$ & $\begin{array}{c}\text { distance } \\
(\mathrm{kpc})\end{array}$ & ref. $^{\mathrm{d}}$ \\
\hline V598 Pup 2007\#2 & $\ldots$ & - & - & - & 11.7 & 12.0 & $-(-)$ & 0.27 & $2.1 \pm 0.2^{\mathrm{e}}$ & 1 \\
\hline V382 Vel 1999 & $\ldots$ & 6.6 & 12.4 & 2.6 & 11.4 & 11.5 & $11.6(11.4)$ & 0.62 & $1.5 \pm 0.1$ & 2 \\
\hline V4743 Sgr 2002\#3 & $\ldots$ & 9.4 & 17 & 5.0 & 13.8 & 13.6 & $13.7(13.7)$ & 0.78 & $3.8 \pm 0.2$ & 3 \\
\hline V1281 Sco $2007 \# 2$ & $\ldots$ & 9.5 & 16.7 & 9.2 & 17.8 & 17.5 & $17.9(17.9)$ & 2.17 & $13 \pm 1$ & 4 \\
\hline V597 Pup 2007\#1 & $\ldots$ & 8.7 & 16.5 & 8.4 & 16.9 & 16.7 & $17.1(17.1)$ & 0.93 & $16 \pm 2$ & 5 \\
\hline V1494 Aql 1999\#2 & $\ldots$ & 11.7 & 22.1 & 5.0 & 13.4 & 13.6 & $13.4(13.6)$ & 1.83 & $2.2 \pm 0.2$ & 6 \\
\hline V2467 Cyg 2007 & $\ldots$ & 12.0 & 23.0 & 7.9 & 16.3 & 16.4 & $16.2(16.5)$ & 4.65 & $2.2 \pm 0.2$ & 7 \\
\hline V5116 Sgr $2005 \# 2$ & $\ldots$ & 15.6 & 28.5 & 7.9 & 16.2 & 16.2 & $16.0(16.2)$ & 0.81 & $12 \pm 1$ & 8 \\
\hline V574 Pup 2004 & $\ldots$ & 18.6 & 35.0 & 7.6 & 15.4 & 15.5 & $15.5(15.7)$ & 2.2 & $4.6 \pm 0.3$ & 8 \\
\hline V458 Vul 2007 & $\begin{array}{l}\cdots \\
\cdots\end{array}$ & 20.7 & 38.7 & 9.4 & 17.0 & 17.0 & $17.2(17.3)$ & 1.86 & $11 \pm 1$ & 9 \\
\hline V1974 Cyg 1992 & $\ldots$ & 16 & 26 & 4.2 & - & {$[12.3]^{\mathrm{f}}$} & 12.5) & 1.00 & 1.8 & 10 \\
\hline V1668 Cyg 1978 & $\begin{array}{l}\cdots \\
\ldots\end{array}$ & 14.4 & 26 & 6.2 & - & {$[14.3]$} & $14.4(14.6)$ & 1.24 & 4.1 & 11 \\
\hline V1500 Cyg 1975 & $\ldots$ & 7.2 & 13 & 3.6 & - & {$[12.5]$} & $12.6(12.4)$ & 1.60 & $1.5^{\mathrm{g}}$ & 12 \\
\hline GK Per 1901 & $\ldots$ & 8.1 & 14.5 & 0.5 & - & {$[9.2]$} & $9.3(9.3)$ & 0.93 & $0.455^{\mathrm{g}}$ & 13 \\
\hline
\end{tabular}

a distance modulus estimated from direct fitting (FF) with the calibrated free-free model light curve

${ }^{b}$ distance modulus estimated from two light curve (LC) fitting

c distance modulus estimated from Schmidt-Kaler's law of Equation 38 or Della Valle \& Livio's law of Equation 39] in parenthesis, i.e., from the Maximum Magnitude-Rate of Decline (MMRD)

d reference for quoted values of $A_{V}$ or $E(B-V)$, where we assume that $A_{V}=3.1 E(B-V)$ : 1- Read et al. (2008), 2. Shore et al. 2003),

3.Vanlandingham et al. (2007), 4 Russell et al. (2007a), 5. Ness et al. (2008c), 6 [ijima \& Esenoglu (2003), 7. Mazuk et al. [2007), 8. Burlak (2008), 9.Wesson et al. [2008), 10 Chochol et al. (1993), 11-Stickland et al. (1981), 12. Downes \& Duerbeck (2000), 13Wu et al. (1989)

e error means a standard deviation of the four distance estimates, i.e., FF, LC, and two MMRDs

$\mathrm{f}$ parenthesis means the distance estimated from other method, see text for details

$\mathrm{g}$ the distance is taken from Downes \& Duerbeck 2000

$$
\log T_{p h}(k e V)
$$

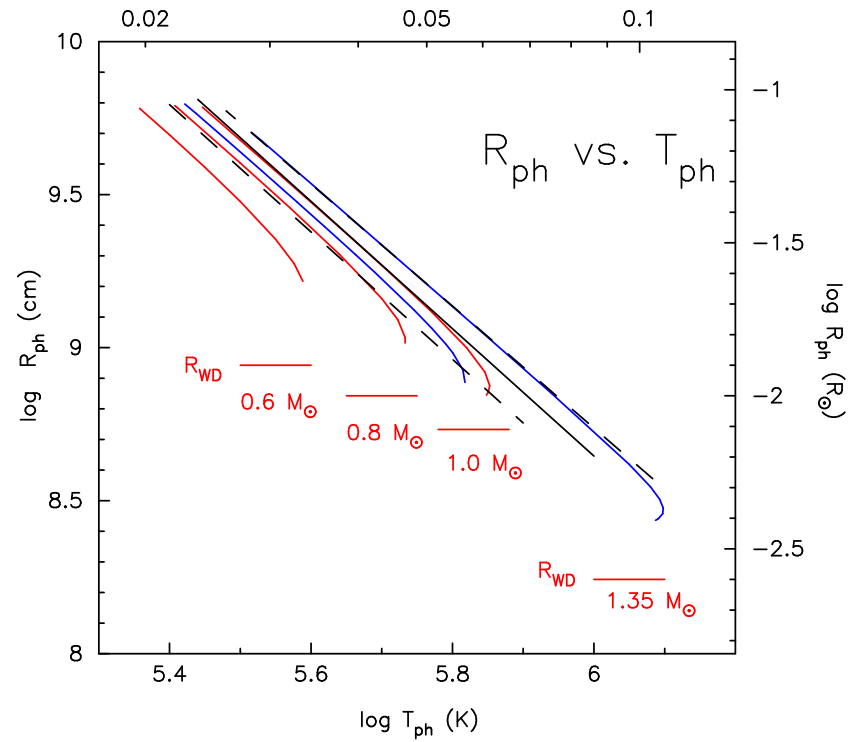

FIG. 29.- Photospheric radius against photospheric temperature for WDs with various masses only during the static phase (after the optically thick winds stop). Red solid lines denote those for $0.6,0.8$, and $1.0 M_{\odot}$ WDs with the chemical composition of "CO nova 2." Blue solid lines denote those for 1.0 and $1.35 M_{\odot}$ WDs with the chemical composition of "Ne nova 2." Black thick solid line indicate an approximate formula of Equation [B1]. Its \pm 0.1 upper/lower bounds are indicated by two black dashed lines. Short horizontal lines indicate the WD radius (i.e., the bottom of a nuclear burning region) of $0.6,0.8,1.0$ and $1.35 M_{\odot}$ WDs. See text in Appendix B for more details.

2. Our free-free emission model light curves reasonably well reproduce optical and near IR light curves of novae. The decay timescale of our free-free emission model light curves depends strongly on the WD mass but weakly on the chemical composition. So the nova speed class is mainly determined by the WD mass.
3. The peak brightness of our model light curve depends also on the initial envelope mass $\left(M_{\text {env, } 0}\right)$ even if we fix the WD mass and the chemical composition of the envelope. This indicates that nova speed class, i.e., $t_{2}$ and $t_{3}$ times, depends also on the initial envelope mass, in other words, the mass accretion rate to the WD.

4. Our UV $1455 \AA$ model light curves for different WD masses are homologous, that is, they are almost overlapped to each other if we stretch them in the direction of time $(t)$ by a factor of $1 / f_{\mathrm{s}}$ and properly normalize the flux. All the free-free emission model light curves for different WD masses are also homologous and overlapped with each other if we rescale them as $t^{\prime}=t / f_{\mathrm{s}}$ in the time and $F_{\nu}^{\prime}=f_{\mathrm{s}} F_{\nu}$ in the flux (see Figures 3 and 6 ), that is, they are overlapped to each other in the $t^{\prime}-m_{V}^{\prime}$ plane, where $m_{V}^{\prime}=-2.5 \log F_{\nu}^{\prime}+$ const. The more massive the WD, the smaller the timescaling (or stretching) factor of $f_{\mathrm{s}}$. We call this "a universal decline law."

5. Using the universal decline law, the apparent magnitudes of various WD mass models are restored by $m_{V}=m_{V}^{\prime}+2.5 \log f_{\mathrm{s}}$ and their absolute magnitudes are calculated from $M_{V}=m_{V}^{\prime}+2.5 \log f_{\mathrm{s}}-(m-M)_{V}$. Therefore, we can determine the absolute magnitudes of freefree emission model light curves by calibrating their absolute magnitudes with the known distance moduli $(m-M)_{V}$ of V1668 Cyg for CO novae and V1974 Cyg for neon novae.

6. Since the absolute magnitudes of our free-free emission model light curves are now calibrated (Tables 2 and 3), we can estimate the distance moduli of individual novae by directly fitting them with observation. We show that the distance moduli thus obtained for V1500 Cyg are consistent with those calculated from the nebular 
expansion parallax method. This is a new method for obtaining the distance modulus to a nova.

7. It has been empirically proposed that the absolute $V$ magnitude 15 days after optical maximum, $M_{V}(15)$, is almost the same among various novae. We show that there is only a small scatter of $0.1-0.3$ mag for the absolute brightnesses of our model light curves 15 days after maximum (see Figures 4 and 7). This results theoretically support the above empirical relation on $M_{V}(15)$.

8. We found that, when the timescales of two nova light curves are similar, the absolute brightnesses of the two novae are almost the same during the period in which the universal decline law is applied. Therefore, we are also able to estimate the distance modulus of a nova by comparing it with the other nova the distance of which is known and the timescale of which is similar. We also show that the distance moduli thus obtained for GK Per are consistent with those calculated from the nebular expansion parallax method. This is another new method for obtaining the distance modulus to a nova.

9. Our free-free model light curve reasonably follows the observed light curve when free-free emission dominates the continuum flux in novae. Even when the nova has a transition oscillation or a characteristic secondary peak, our free-free emission model light curve can be fitted with the bottom line of observed nova light curves (see Figures 10, 11, and 26).

The Second part contains timescales of novae, our prediction formulae of supersoft X-ray on and off times, and our theoretical MMRD relations, which are derived from the universal decline law:

10. We derive several relations between the characteristic timescales of classical novae, i.e., $t_{\text {break }}, t_{\text {wind }}$, and

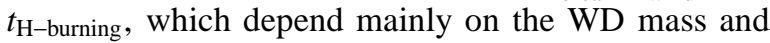
weakly on the chemical composition but almost independent of the initial envelope mass.

11. On the other hand, $t_{2}$ and $t_{3}$ depend sharply on the initial envelope mass. Adopting a typical (average) case of the initial envelope mass, we obtain "average" relations between $t_{3}$ (or $t_{2}$ ) time and the three timescales of $t_{\text {break }}, t_{\text {wind }}$, and $t_{\mathrm{H} \text {-burning. These proposed relations }}$ are reasonably fitted with published data of novae. We propose the relation between the "intrinsic" $t_{3}$ time and $t_{\text {wind }}$, i.e., Equation (30), as a prediction formula for the start of a supersoft X-ray phase, and the relation between the "intrinsic" $t_{3}$ and $t_{\mathrm{H}-\text { burning }}$, i.e., Equation (31), as a prediction formula for the end of it. Here we propose the "intrinsic" $t_{3}$ time which is calculated along our free-free emission model light curve fitted with the observation.

12. We obtain a relation between the maximum magnitude, $m_{V, \text { max }}=m_{V, \text { max }}^{\prime}+2.5 \log f_{\mathrm{s}}$, and the rate of decline, $\log t_{3}=\log \left(t_{3}^{\prime} f_{\mathrm{s}}\right)$, through the stretching factor $f_{\mathrm{s}}$ of the free-free emission model light curves, i.e., Equation (32). A more massive WD corresponds to a smaller stretching factor of $f_{\mathrm{s}}$ while the initial envelope mass determines a set of $\left(t_{3}^{\prime}, m_{V, \text { max }}^{\prime}\right)$. Calibrating the distance moduli $(m-M)_{V}$ of V1668 Cyg and V1974 Cyg, we derive usual MMRD relations between the absolute maximum magnitude $M_{V, \max }$ and $\log t_{3}$ by changing $f_{\mathrm{s}}$ for a given set of $\left(t_{3}^{\prime}, m_{V, \max }^{\prime}\right)$, i.e., Equation (34).

13. Our MMRD relations theoretically confirm that the main parameter is the WD mass, $M_{\mathrm{WD}}$. A more massive WD with a smaller $f_{\mathrm{s}}$ corresponds to a faster nova, i.e., a shorter $t_{3}$ time. However, there is a second parameter, i.e., the initial envelope mass $M_{\text {env, } 0}$, corresponding to $\left(t_{3}^{\prime}, m_{V, \max }^{\prime}\right)$. The larger the envelope mass, the brighter the maximum magnitude, and the shorter the rate of decline . The main parameter $M_{\mathrm{WD}}$ produces a main trend of MMRD relation and the second parameter $M_{\mathrm{env}, 0}$ can reasonably explain the scatter around the main MMRD relation.

14. Our MMRD relations are also in good agreement with Kaler-Schmidt's law (Schmidt 1957) and Della Valle \& Livio's (1995) law at their central lines (values).

The last part concerns light-curve fitting of ten classical novae in which a supersoft X-ray phase was detected:

15. The universal decline law reasonably reproduces the observed light curves of ten classical novae, in which a supersoft X-ray phase was detected. The models reproducing simultaneously the optical and supersoft Xray observations are WDs with $1.28 \pm 0.04 M_{\odot}(\mathrm{V} 598$ Pup), $1.23 \pm 0.05 M_{\odot}(\mathrm{V} 382 \mathrm{Vel}), 1.15 \pm 0.06 M_{\odot}$ (V4743 Sgr), $1.13 \pm 0.06 M_{\odot}(\mathrm{V} 1281 \mathrm{Sco}), 1.2 \pm$ $0.05 M_{\odot}$ (V597 Pup), $1.06 \pm 0.07 M_{\odot}(\mathrm{V} 1494 \mathrm{Aql})$, $1.04 \pm 0.07 M_{\odot}(\mathrm{V} 2467 \mathrm{Cyg}), 1.07 \pm 0.07 M_{\odot}(\mathrm{V} 5116$ Sgr), $1.05 \pm 0.05 M_{\odot}$ (V574 Pup), $0.93 \pm 0.08 M_{\odot}$ (V458 Vul). The first nine WDs are probably ONeMg WDs (ONe novae) while the last one is probably a CO WD (CO nova). Our light curve fittings also suggest various physical values of novae, such as the envelope mass of $M_{\text {env. } 0}$ at optical maximum, the ejecta mass of $M_{\text {wind }}$, etc. as tabulated in Table 7

16. The newly proposed relationships of Equations (30) and (31) are consistent with the emergence/decay epoch of the supersoft X-ray phase of these novae including V1974 Cyg.

17. We estimate distances to the ten classical novae by using our two new methods and show that they are consistent with those derived from the MMRD relation based on the "intrinsic" $t_{3}$ time as tabulated in Table 8 .

18. We propose a mechanism of early hard X-ray emission originated from the shock between the optically thick winds and the companion star. Our model predicts that the hard X-rays emerge when the companion appears from the white dwarf photosphere and decay when the optically thick winds stop. Our estimates of emerging-time $t_{\text {emerge }}$ of the companion from the nova photosphere are consistent with the observed hard Xray appearance in V382 Vel, V1494 Aql, and V458 Vul; which may support our idea of hard X-ray emission.

We thank AAVSO, AFOEV, VSNET, and VSOLJ for nova photometric data. We are also grateful to the anonymous referee for useful comments that helped to improve the paper. 
This work made use of data supplied by the UK Swift Science Data Centre at the University of Leicester. This research has been supported in part by the Grant-in-Aid for Scientific
Research (20540227) of the Japan Society for the Promotion of Science.

\section{APPENDIX}

\section{SCALING LAW OF BRIGHTNESS AND ABSOLUTE FLUX OF FREE-FREE EMISSION LIGHT CURVES}

In this appendix, we present a formulation of scaling law for free-free emission model light curves and derive Equation (5). We determine the flux of our free-free light curves against that of $0.95 M_{\odot}$ WD $(\approx \mathrm{V} 1668$ Cyg $)$. Now we explicitly express the proportionality constant in Equation (1) as

$$
F_{\nu}^{\left\{M_{\mathrm{WD}}\right\}}(t)=C^{\left\{M_{\mathrm{WD}}\right\}}\left[\frac{\dot{M}_{\mathrm{wind}}^{2}}{v_{\mathrm{ph}}^{2} R_{\mathrm{ph}}}\right]_{(t)}^{\left\{M_{\mathrm{WD}}\right\}} .
$$

In free-free emission, $F_{\nu}$ is independent of frequency $\nu$, then $C^{\left\{M_{\mathrm{WD}}\right\}}$ is also independent of frequency because each term in the square bracket of the right-hand-side is also independent of frequency. The proportionality constant $C^{\left\{M_{\mathrm{WD}}\right\}}$ probably depends not only on the WD mass but also weakly on the chemical composition and other physical conditions. However, we here assume that $C^{\left\{M_{\mathrm{WD}}\right\}}$ depends only on $M_{\mathrm{WD}}$ when we fix the chemical composition.

Then, we obtained $F_{\nu^{\prime}}^{\prime}=f_{\mathrm{s}} F_{\nu}$ from Equation (4) after time-stretching $\left(t^{\prime}=t / f_{\mathrm{s}}\right.$ and $\left.\nu^{\prime}=f_{\mathrm{s}} \nu\right)$. Since the free-free flux is independent of the frequency $\nu$, i.e., $F_{\nu^{\prime}}^{\prime}=F_{\nu}^{\prime}$, this simply means

$$
F_{\nu}^{\left\{0.95 M_{\odot}\right\}}(t)=f_{\mathrm{s}} F_{\nu}^{\left\{M_{\mathrm{WD}}\right\}}\left(t / f_{\mathrm{s}}\right) .
$$

Now, we define the apparent $V$ magnitudes as

$$
m_{V}(t) \equiv-2.5 \log F_{\nu}^{\left\{M_{\mathrm{WD}}\right\}}(t)+H_{V}
$$

and, for $0.95 M_{\odot}$ WD $(\approx \mathrm{V} 1668 \mathrm{Cyg})$ with $f_{\mathrm{s}} \approx 1$, we have

$$
m_{V}^{\prime \prime}(t) \equiv-2.5 \log F_{\nu}^{\left\{0.95 M_{\odot}\right\}}(t)+H_{V}
$$

where we introduce a constant $H_{V}$ in order to fit $m_{V}^{\prime \prime}$ of $0.95 M_{\odot}$ WD model with the $V$ observation of V1668 Cyg, and $H_{V}$ is the same among various WD masses. Using Equation (A2), we have

$$
m_{V}^{\prime \prime}(t)=m_{V}\left(t / f_{\mathrm{s}}\right)-2.5 \log f_{\mathrm{s}} .
$$

By the way, we have already defined the apparent $V$ magnitudes of our free-free emission model light curves as

$$
m_{V}^{\prime}\left(t / f_{\mathrm{s}}\right) \equiv-2.5 \log \left[\frac{\dot{M}_{\mathrm{wind}}^{2}}{v_{\mathrm{ph}}^{2} R_{\mathrm{ph}}}\right]_{\left(t / f_{\mathrm{s}}\right)}^{\left\{M_{\mathrm{WD}}\right\}}+K_{V},
$$

in Equation (6). Here $K_{V}$ is a constant defined to fit $m_{V}^{\prime}$ of $0.95 M_{\odot}$ WD model with the $V$ observation of V1668 Cyg, and common among all the WD mass models (from $M_{\mathrm{WD}}=0.55$ to $1.2 M_{\odot}$ by $0.05 M_{\odot}$ step) for a chemical composition of "CO nova 2." We saw that all the light curves are almost overlapped to each other in Figure 2. This simply means that

$$
\left[\frac{\dot{M}_{\mathrm{wind}}^{2}}{v_{\mathrm{ph}}^{2} R_{\mathrm{ph}}}\right]_{\left(t / f_{\mathrm{s}}\right)}^{\left\{M_{\mathrm{wD}}\right\}} \approx\left[\frac{\dot{M}_{\mathrm{wind}}^{2}}{v_{\mathrm{ph}}^{2} R_{\mathrm{ph}}}\right]_{(t)}^{\left\{0.95 M_{\odot}\right\}} .
$$

Substituting Equation (A1) into Equation (A2) and using Equation (A7), we have

$$
C^{\left\{0.95 M_{\odot}\right\}} \approx f_{\mathrm{s}} C^{\left\{M_{\mathrm{WD}}\right\}} .
$$

From Equation (A7) together with Equations (A4) and (A6), we derive

$$
m_{V}^{\prime}\left(t / f_{\mathrm{s}}\right)-K_{V} \approx m_{V}^{\prime \prime}(t)+2.5 \log C^{\left\{0.95 M_{\odot}\right\}}-H_{V} .
$$

Therefore, we may regard that $m_{V}^{\prime \prime}(t)=m_{V}^{\prime}\left(t / f_{\mathrm{s}}\right)$, or in other words,

$$
m_{V}^{\prime}\left(t / f_{\mathrm{s}}\right)=m_{V}\left(t / f_{\mathrm{s}}\right)-2.5 \log f_{\mathrm{s}},
$$

if we define $K_{V}$ as

$$
K_{V}=H_{V}-2.5 \log C^{\left\{0.95 M_{\odot}\right\}} .
$$

Thus, we derive Equation (5). Therefore, we plotted all the brightnesses of novae in the $t^{\prime}=t / f_{\mathrm{s}}$ vs. $m_{V}^{\prime}=m_{V}-2.5 \log f_{\mathrm{s}} \operatorname{diagram}$ as shown in Figure 2 .

It should be noted that the UV $1455 \AA$ and supersoft X-ray model light curves follow $F_{\nu^{\prime}}^{\prime}=f_{\mathrm{s}} F_{\nu}$ after stretching but do not satisfy $F_{\nu^{\prime}}=F_{\nu}$, so that these fluxes do not obey Equations (A2) and (A5), because the blackbody emissivity depends on the frequency $\nu$ and, as a result, the spectrum changes after time-stretching $\left(\nu^{\prime}=f_{\mathrm{s}} \nu\right)$. Therefore, we have to normalize the UV fluxes by different factors to fit the peaks with the observational peak of V1668 Cyg, as already mentioned in Section 3 


\section{AN EMPIRICAL FORMULA OF WHITE DWARF PHOTOSPHERIC RADIUS}

The photospheric radius of a typical nova during a supersoft X-ray phase is usually much larger than the Chandrasekhar radius. It is about ten times larger $\left(\sim 6 \times 10^{9} \mathrm{~cm}\right)$ than the Chandrasekhar radius at the beginning of a supersoft X-ray phase and quickly shrinks with the photospheric temperature being increasing. Therefore, we here present a simple formula on the radius of WD photosphere during a supersoft X-ray phase, which may be used for estimating the X-ray emission area. The photospheric radius of the WD envelope in the supersoft X-ray phase is plotted in Figure 29 against the photospheric temperature for various WD masses with the chemical composition of "CO nova 2" and "Ne nova 2." The left edge of each line corresponds to the epoch when the wind stops (at $t_{\text {wind }} \approx t_{\mathrm{X}-\mathrm{on}}=$ when the supersoft X-ray emerges). As the photosphere shrinks, the photospheric temperature rises. The rightmost point of each line denotes the epoch when hydrogen nuclear burning stops. We see that the photospheric radius $R_{\mathrm{ph}}$ is almost inversely proportional to a square of the photospheric temperature $T_{\mathrm{ph}}$, i.e., $R_{\mathrm{ph}} \propto 1 / T_{\mathrm{ph}}^{2}$, because the total luminosity is almost constant, $L_{\mathrm{ph}}=4 \pi R_{\mathrm{ph}}^{2} \sigma T_{\mathrm{ph}}^{4} \sim$ const., in the supersoft X-ray phase except the very later phase.

The radius of a typical fast classical nova (1.0 $M_{\odot}$ WD with a composition of "CO nova 2 ") can be approximated by the black solid line as

$$
\log R_{\mathrm{ph}}(\mathrm{cm})=-2.07\left(\log T_{\mathrm{ph}}(\mathrm{K})-5.7\right)+9.27
$$

for $5.45<\log T_{\mathrm{ph}}(\mathrm{K})<5.8$. The dashed lines denote the upper and lower bound values of $\log R_{\mathrm{ph}} \pm 0.1$ which covers the most of the WD mass representative for classical novae, i.e., from $0.8 M_{\odot}$ with "CO nova 2" to $1.35 M_{\odot}$ with "Ne nova 2." The slow novae are not covered in the region, i.e., 0.6-0.7 $M_{\odot}$ with "CO novae 2" has smaller photospheric radius as shown in Figure 29 This figure also shows the WD radius, i.e., the bottom of a hydrogen burning zone: the Chandrasekhar radius for $M_{\mathrm{WD}}<1.33 M_{\odot}$ and values taken from Nomoto et al. (1984) for $M_{\mathrm{WD}} \geq 1.33 M_{\odot}$.

\section{REFERENCES}

Arkhipova, V. P., Burlak, M. A., \& Esipov, V. F. 2002, Astronomy Letters, 28,100

Augusto, A., \& Diaz, M. P. 2003, AJ, 125, 3349

Balman, Ş., Retter, A., \& Bos, M. 2006, AJ, 131, 2628

Barsukova, E. A., \& Goranskii, V. P. 2003, Astronomy Letters, 29, 195

Bonifacio, P., Selvelli, P. L., \& Caffau, E. 2000, A\&A, 356, L53

Bos, M., Retter, A., Cook, L., \& Novak, R. 2001, IAU Circ., 7665, 2

Brown, N., \& Pearce, A. 2002, IAU Circ., 7982, 3

Burlak, M. A. 2008, Astronomy Letters, 34, 249

Burlak, M. A., \& Henden, A. A. 2008, Astronomy Letters, 34, 241

Burwitz, V., Starrfield, S., Krautter, J., \& Ness, J.-U. 2002, AIP Conf. Proc. Vol. 637, Classical Nova Explosions, eds. M. Hernanz \& J. José (AIP, New York), 377

Buscombe, W., \& de Vaucouleurs, G. 1955, The Observatory, 75, 170

Capaccioli, M., della Valle, M., Rosino, L., D’Onofrio, M. 1989, AJ, 97, 1622

Capaccioli, M., Della Valle, M., D’Onofrio, M., Rosino, L. 1990, ApJ, 360, 63

Cassatella, A., Altamore, A., \& González-Riestra, R. 2002, A\&A, 384, 1023

Cassatella, A., Lamers, H. J. G. L. M., Rossi, C., Altamore, A. González-Riestra, R. 2004, A\&A, 420, 571

Chochol, D., Grygar, J., Pribulla, Komzik, R., Hric, L., \& Elkin, V. 1997, A\&A, 318, 908

Chochol, D., Hric, L., Urban, Z., Komzik, R., Grygar, J., \& Papousek, J. 1993, A\&A, 277, 103

Cohen, J. G. 1985, ApJ, 292, 90

Cohen, J. G. 1988, The extragalactic distance scale, ASP conference series vol. 4 (Astron. Soc. Pacific: San Francisco), 114

Della Valle, Massimo; Livio, M. 1995, ApJ, 452, 704

Della Valle, M., Pasquini, L., Daou, D., \& Williams, R. E. 1999, IAU Circ., 7194,1

Della Valle, M., Pasquini, L., Daou, D., \& Williams, R. E. 2002, A\&A, 390, 155

de Vaucouleurs, G. 1978, ApJ, 223, 351

Dobrotka, A., Friedjung, M., Retter, A., Hric, L., Novak, R. 2006, A\&A, 448,1107

Dobrotka, A., Retter, A., \& Liu, A. 2008, A\&A, 478, 815

Downes, R. A., \& Duerbeck, H. W. 2000, AJ, 120, 2007

Drake, J. J. et al. 2007, The Astronomer's Telegram, 1246

Drake, J. J. et al. 2008, The Astronomer's Telegram, 1721

Drake, J. J. et al. 2003, ApJ, 584, 448

Duerbeck, H. W. 1999, Inf. Bul. Var. Stars, 4731, 1

Ennis, D., Beckwith, S., Gatley, I., Matthews, K., Becklin, E. E., Elias, J., Neugebauer, G., \& Willner, S. P. 1977, ApJ, 214, 478

Evans, et al. 2009, MNRAS, submitted (arXiv: 0812.3662)

Eyres, S. P. S., Heywood, I., O'Brien, T. J., Ivison, R. J., Muxlow, T. W. B., \& Elkin, V. G. 2005, MNRAS, 358, 1019

Ferland, G. J., Lambert, D. L., \& Woodman, J. H. 1986, ApJS, 60, 375

Friedjung, M. 1987, A\&A, 180, 155
Fujii, M., et al. 1999, IAU Circ., 7324, 1

Gallagher, J. S., \& Ney, E. P. 1976, ApJ, 204, L35

Gallagher, J. S., Kaler, J. B., Olson, E. C., Hartkopf, W. I., \& Hunter, D. A. 1980, PASP, 92, 46

Gilmore, A. C. 1999, IAU Circ., 7179, 1

Goranskij, V. P., Metlova, N. V., Barsukova, E. A., Burenkov, A. N.,

Soloviev, V. Ya. 2008, The Astronomer's Telegram, 1631

Hachisu, I., \& Kato, M. 2001, ApJ, 558, 323

Hachisu, I., \& Kato, M. 2003, ApJ, 590, 445

Hachisu, I., \& Kato, M. 2004, ApJ, 612, L57

Hachisu, I., \& Kato, M. 2005, ApJ, 631, 1054

Hachisu, I., \& Kato, M. 2006, ApJS, 167, 59

Hachisu, I., \& Kato, M. 2007, ApJ, 662, 552

Hachisu, I., \& Kato, M. 2009, ApJ, 694, L103

Hachisu, I., Kato, M., \& Cassatella, A. 2008, ApJ, 687, 1236

Hachisu, I., et al. 2006, ApJ, 651, L141

Hachisu, I., Kato, M., \& Luna, G. J. M. 2007, ApJ, 659, L153

Haseda, K., West, D., Yamaoka, H., \& Masi, G. 2002, IAU Circ., 7975, 1

Heywood, I., O’Brien, T. J., Eyres, S. P. S., Bode, M. F., \& Davis, R. J.

2005, MNRAS, 362, 469

Hjellming, R. M., van Gorkom, J. H., Taylor, A. R., Seaquist, E. R., Padin,

S., Davis, R. J., \& Bode, M. F. 1986, ApJ, 305, L71

Iijima, T. 2006, A\&A, 451, 563

Iijima, T., \& Esenoglu, H. H. 2003, A\&A, 404, 997

Kaler, J. B. 1986, PASP, 98, 243

Kamath, U. S., Anupama, G. C., Ashok, N. M., Mayya, Y. D., Sahu, D. K. 2005, MNRAS, 361, 1165

Kang, T. W., Retter, A., Liu, A., \& Richards, M. 2006, AJ, 132, 608

Kato, M., \& Hachisu, I., 1994, ApJ, 437, 802

Kato, M., \& Hachisu, I., 2007, ApJ, 657, 1004

Kato, M., Hachisu, I., \& Cassatella, A. 2009, ApJ, 704, 1676

Kato, T., et al. 2002, IAU Circ., 7976, 2

Kawara, K., Maihara, T., Noguchi, K., Oda, N., Sato, S., Oishi, M., \& Iijima, T. 1976 , PASJ, 28 , no. 1,1976 , p. 163

Kiss, L. L., \& Thomson, J. R. 2000, A\&A, 355, L9

Krautter, J., Beuermann, K., Leitherer, C., Oliva, E., Moorwood, A. F. M., Deul, E., Wargau, W., Klare, G., Kohoutek, L., van Paradijs, J., \& Wolf, B. 1984, A\&A, 37, 307

Krautter, J., Ögelman, H., Starrfield, S., Wichmann, R., \& Pfeffermann, E. 1996, ApJ, .456, 788

Lance, C. M., McCall, M. L., \& Uomoto, A. K. 1988, ApJS, 66, 151

Liller, W. 2005, IAU Circ., 8559, 1

Liller, W., \& Jones, A. 2000, Inf. Bul. Var. Stars, 5004, 1.

Lockwood, G. W., \& Millis, R. L. 1976, PASP, 88, 235

Lynch, D. K., Russell, R. W., Rudy, R. J., \& Woodward, C. E. 2008,

IAU Circ., 8883, 1

Lynch, David K., Russell, R. W., Rudy, R. J., \& Woodward, C. E. 2009,

American Astronomical Society, AAS Meeting 213, 491.25

Mallama, A. D., \& Skillman, D. R. 1979, PASP, 91, 99 
Mazuk, S., Lynch, D. K., Rudy, R. J., Russell, R. W., Pearson, R. L., Woodward, C. E., \& Puetter, R. C. 2007, IAU Circ., 8848, 1

Mazuk, S., Rudy, R. J., Lynch, D. K., Venturini, C., Puetter, R. C., Armstrong, T. 2000, IAU Circ., 7490, 3

Morgan, G. E.; Ringwald, F. A.; Prigge, J. W. 2003, MNRAS, 344, 521

Mukai, K., \& Ishida, M. 2001, ApJ, 551, 1024

Munari, U., Dalla Via, G., Valisa, P., Dallaporta, S., \& Castellani, F. 2007, CBET, 897

Munari, U., Henden, A., Pojmanski, G., Dallaporta, S., Siviero, A., \& Navasardyan, H. 2006a, MNRAS, 369, 1755

Munari, U., et al. 2008, A\&A, 492, 145

Munari, U., Siviero, A., Navasardyan, H., \& Dallaporta, S. 2006b, A\&A, 452,567

Naik, S., Banerjee, D. P. K., Ashok, N. M. 2009, MNRAS, 394, 1551

Naito, H., Narusawa, S., Yamaoka, H., \& Fujita, Y. 2007, IAU Circ., 8812, 2

Nakano, S., Kadota, K., Waagen, E., Swierczynski, S., Komorous, M., King, R., \& Bortle, J. 2007a, IAU Circ., 8861, 1

Nakano, S., Tago, A., Nishiyama, K., \& Sakamoto, T. 2007b, IAU Circ., 8821,1

Nakano, S., Tago, A., Sakurai, Y., Kushida, R., \& Kadota, K. 2004, IAU Circ., 8443, 1

Nelson, T., et al. 2007, The Astronomer's Telegram, 1202

Ness, J.-U., et al. 2003, ApJ, 594, L127

Ness, J.-U., et al. 2009, AJ, 137, 4160

Ness, J.-U.; Schwarz, G.; Osborne, J. P.; Page, K.; Drake, J. J., \& Krautter, J. 2008b, The Astronomer's Telegram, 1747, 1

Ness, J.-U., Schwarz, G. J., Retter, A., Starrfield, S., Schmitt, J. H. M. M., Gehrels, N., Burrows, D., \& Osborne, J. P. 2007a, ApJ, 663, 505

Ness, J.-U., Starrfield, S., Jordan, C., Krautter, J., \& Schmitt, J. H. M. M. 2005, MNRAS, 364, 1015

Ness, J.-U., Starrfield, S., Schwarz, G., Bode, M. F., Osborne, J. P., \& Page, K. L. 2008a, The Astronomer's Telegram, 1370

Ness, J.-U., Starrfield, S., Schwarz, G., Osborne, J. P., \& Page, K. L. 2007b, The Astronomer's Telegram, 1327, 1

Ness, J.-U., et al. 2008c, IAU Circ., 8911, 2

Ness, J.-U., Starrfield, S., Schwarz, G., Page, K. L., \& Osborne, J. P. 2007c, CBET, 1030, 1

Nielbock, M., \& Schmidtobreick, L. 2003, MNRAS, 400, L5

Nomoto, K. 1982, ApJ, 253, 798

Nomoto, K., Thielemann, F., \& Yokoi, K. 1984, ApJ, 286, 644

Novak, R., Retter, A., Cook, L., Korcakova, D., \& Janik, J. 2000, IAU Circ., 7448, 4

Orio, M., Covington, J., Ögelman, H. 2001a, A\&A, 373, 542

Orio, M., et al. 2003, IAU Circ., 8131, 2

Orio, M., \& Tepedelenlioglu, E. 2003, IAU Circ., 8435, 2

Orio, M., Parmar, A. N., Greiner, J., Ögelman, H., Starrfield, S., \& Trussoni, E. 2002, MNRAS, 333, L11

Orio, M., et al. 2001b, MNRAS, 326, L13

Pereira, A., di Cicco, D., Vitorino, C., \& Green, D. W. E. 1999, IAU Circ., 7323, 1

Pereira, A. J. S., McGaha, J. E., Young, J., \& Rhoades, H. 2007, IAU Circ., 8895, 1

Poggiani, R. 2008, Ap\&SS, 315, 79

Poggiani, R. 2009, Astron. Nachr., 330, 77

Pojmanski, G., Szczygiel, D., \& Pilecki, B. 2007, IAU Circ. 8899.

Prialnik, D., \& Kovetz, A. 1995, ApJ, 445, 789

Read, A. M. et al. 2008, A\&A, 482, L1

Read, A. M. et al. 2007, The Astronomer's Telegram, No. 1301

Retter, A., Cook, L., Novak, R., Saxton, J. M., Jensen, L. T., Korcakova, D., \& Janik, J. 2000, IAU Circ., 7537, 2

Rohrbach, J. G., Ness, J.-U., \& Starrfield, S. 2009, AJ, 137, 4627

Rosino, L. 1987, RS Ophiuchi (1985) and the Recurrent Novae

Phenomenon, ed. M. F. Bode (Utrecht, VNU Science Press), 1

Rudy, R. J., et al. 2006, Bull. of the American Astr. Soc., 38, 907
Russell, R. W., Rudy, R. J., Lynch, D. K., Mazuk, S., Pearson, R. L.,

Woodward, C. E., Puetter, R. C., \& Perry, R. B. 2007a, IAU Circ., 8846,

Russell, R. J., Rudy, R. J., Lynch, D. K., \& Woodward, C. E. 2007b, IAU Circ., 8888, 1

Sala, G., Hernanz, M., \& Ferri, C. 2007, The Astronomer's Telegram, 1184

Sala, G., Hernanz, M., Ferri, C., \& Greiner, J. 2008, A\&A, 675, L93

Schmidt, Th. 1957, Z. Astrophys., 41, 181

Selvelli, P.; Friedjung, M. 2003, A\&A, 401, 297

Senziani, F., Skinner, G. K., Jean, P., \& Hernanz, M. 2008, A\&A, 485, 223

Shanley, L., Ögelman, H., Gallagher, J. S., Orio, M., \& Krautter, J. 1995, ApJ, 438, L95

Shore, S. N. et al. 2003, AJ, 125, 1507

Siviero, A., Munari, U., \& Jones, A. F. 2005, Inf. Bul. Var. Stars, 5638, 1

Slavin, A. J., O’Brien, T. J., \& Dunlop, J. S. 1995, MNRAS, 276, 353

Snijders, M. A. J. 1987a, RS Ophiuchi (1985) and the Recurrent Novae

Phenomenon, ed. M. F. Bode (Utrecht, VNU Science Press), 51

Snijders, M. A. J. 1987b, Ap\&SS, 130, 243

Sostero, G., Yamaoka, H., Kiyota, S., Nakajima, K., \& Maehara, H. 2004, IAU Circ., 8445, 3

Starrfield, S., Ness, J.-U., Drake, J. J., \& Orio, M. 2003, IAU Circ., 8107, 1

Steiner, J. E., \& Campos, R 1999, IAU Circ., 7185, 2

Stickland, D. J., Penn, C. J., Seaton, M. J., Snijders, M. A. J., \& Storey, P. J. 1981, MNRAS, 197, 107

Swierczynski, E., Ragan, E., Galan, C., \& Mikolajewski, M. 2008, The

Astronomer's Telegram, 1723

Tarasova, T. N. 2008, Odessa Astronomical Publications, 21, 120

Tempesti, P. 1979, Astron. Nachricheten, Bd. 300, 51

Tomkin, J., Lambert, D. L., \& Woodman, J. 1976, A\&A, 48, 319

Torres, M. A. P., Jonker, P. G., Challis, P., Modjaz, M., Kirshner, R., Read, A. M., Kuulkers, E., \& Saxton, R. D. 2007, The Astronomer's Telegram, No. 1285

Tomov, T., et al. 2007, Inf. Bul. Var. Stars, 5779, 1

Tsujimoto, M., Takei, D., Drake, J. J., Ness, J.-U., \& Kitamoto, S. 2008, PASJ, 61, S69

Tylenda, R. 1978, Acta Astronomica, 28, 333

Umeda, H., Nomoto, K., Yamaoka, H., \& Wanajo, S. 1999, ApJ, 513, 861

van den Bergh, S., \& Younger, P. F. 1987, A\&AS, 70, 125

Vanlandingham, K. M., Schwarz, G. J., Shore, S. N., \& Starrfield, S. 2001, AJ, 121, 1126

Vanlandingham, K. M., Schwarz, G., Starrfield, S., Woodward, C., Wagner, M., Ness, J., Helton, A. 2007, Bulletin of the American Astronomical Society, 38, 99

Venturini, C. C., Rudy, R. J., Lynch, D. K., Mazuk, S., \& Puetter, R. C. 2004, AJ, 128, 405

Warner, B. 1995, Cataclysmic variable stars, Cambridge, Cambridge University Press

Warner, B., \& Woudt, P. A. 2009, MNRAS, 397, 979

Webbink, R. F., Rappaport, S., \& Savonije, G. J. 1983, ApJ, 270, 678

Wesson, R. et al. 2008, ApJ, 688, L21

Whitelock, P. A., Carter, B. S., Feast, M. W., Glass, I. S., Laney, D.,

Menzies, J. W., Walsh, J., \& Williams, P. M. 1984, MNRAS, 211, 421

Williams, P., \& Gilmore, A. C. 1999, IAU Circular No. 7176, 1

Woodward, C. E., Gehrz, R. D., Jones, T. J., Lawrence, G. F., \& Skrutskie, M. F. 1997, ApJ, 477, 817

Woodward, C. E., Wooden, D. H., Pina, R. K., \& Fisher, R. S. 1999, IAU

Circ., 7220, 3

Woudt, P. A., Warner, B., \& Spark, M. 2005, MNRAS, 364, 107

Wu, C.-C., Holm, A. V., Panek, R. J., Raymond, J. C., Hartmann, L. W., \&

Swank, J. H. 1989, ApJ, 339, 443

Yamaoka, H., Nakamura, Y., Itagaki, K., Nakano, S., \& Nishimura, H. 2007, IAU Circ., 8810, 1 